\title{
HOMOGENIZATION WITH CORRECTOR FOR A STATIONARY PERIODIC MAXWELL SYSTEM
}

\author{
T. A. SUSLINA
}

\begin{abstract}
The homogenization problem in the small period limit for a stationary periodic Maxwell system in $\mathbb{R}^{3}$ is studied. It is assumed that the dielectric permittivity and the magnetic permeability are rapidly oscillating (depending on $\mathbf{x} / \varepsilon$ ), positive definite, and bounded matrix-valued functions. For all four physical fields (the strength of the electric field, the strength of the magnetic field, the electric displacement vector, and the magnetic displacement vector), uniform approximations in the $L_{2}\left(\mathbb{R}^{3}\right)$-norm are obtained with the (order-sharp) error term of order $\varepsilon$. Besides solutions of the homogenized Maxwell system, the approximations contain rapidly oscillating terms of zero order that weakly tend to zero. These terms can be interpreted as correctors of zero order.
\end{abstract}

\section{$\S 0$. INTRODUCTION}

0.1. Statement of the problem. The paper is devoted to the homogenization problem for a stationary periodic Maxwell system in $\mathbb{R}^{3}$. This problem was studied intensively by traditional methods of homogenization theory (see, e.g., $\mathrm{BeLP}, \mathrm{Sa}, \mathrm{BaPa}, \mathrm{ZhKO}$ ). The operator-theoretic approach suggested by M. Sh. Birman and T. A. Suslina in BSu1, BSu2 and developed in the subsequent papers [BSu3- $\mathrm{BSu} 5$ was applied to a stationary periodic Maxwell system in [BSu2, Su1, Su2, and [BSu6, Chapter 7]. We start with a survey of the corresponding results.

Let $L_{2}\left(\mathbb{R}^{3} ; \mathbb{C}^{3}\right)$ be the $L_{2}$-space of $\mathbb{C}^{3}$-valued functions in $\mathbb{R}^{3}$, and let $H^{s}\left(\mathbb{R}^{3} ; \mathbb{C}^{3}\right)$, $s>0$, be the corresponding Sobolev classes. We put

$$
\mathfrak{G}=L_{2}\left(\mathbb{R}^{3} ; \mathbb{C}^{3}\right), \quad J=\{\mathbf{f} \in \mathfrak{G}: \operatorname{div} \mathbf{f}=0\} .
$$

Let $\Gamma$ be a lattice in $\mathbb{R}^{3}$, and let $\Omega$ be the elementary cell of $\Gamma$. Suppose that the dielectric permittivity $\eta(\mathbf{x})$ and the magnetic permeability $\mu(\mathbf{x})$ are positive definite and bounded $\Gamma$-periodic $(3 \times 3)$-matrix-valued functions. We denote by $\mathbf{u}$ the electric field strength and by $\mathbf{v}$ the magnetic field strength; then $\mathbf{w}=\eta \mathbf{u}$ is the electric displacement vector, and $\mathbf{z}=\mu \mathbf{v}$ is the magnetic displacement vector. It is convenient to write the Maxwell operator $\mathcal{M}$ in terms of the displacement vectors, assuming that the vectors $\mathbf{w}$ and $\mathbf{z}$ are divergence-free. Then the operator $\mathcal{M}=\mathcal{M}(\eta, \mu)$ acts in the space $J \oplus J$ and is given by the formula

$$
\mathcal{M}=\left(\begin{array}{cc}
0 & i \operatorname{curl} \mu^{-1} \\
-i \operatorname{curl} \eta^{-1} & 0
\end{array}\right)
$$

on the natural domain (see (7.1) below). The point $\lambda=i$ is a regular point for $\mathcal{M}$.

Let $\varepsilon>0$ be a parameter. We study the family of operators $\mathcal{M}_{\varepsilon}=\mathcal{M}\left(\eta^{\varepsilon}, \mu^{\varepsilon}\right)$ with coefficients $\eta^{\varepsilon}(\mathbf{x})=\eta\left(\varepsilon^{-1} \mathbf{x}\right), \mu^{\varepsilon}(\mathbf{x})=\mu\left(\varepsilon^{-1} \mathbf{x}\right)$, which oscillate rapidly as $\varepsilon \rightarrow 0$. We are

2000 Mathematics Subject Classification. Primary 35P20, 35Q60.

Key words and phrases. Periodic Maxwell operator, homogenization, effective medium, corrector.

Supported by RFBR (grant no. 05-01-01076-a) and the President grant "Scientific Schools" (grant no. 5403.2006.1). 
interested in the behavior of the resolvent $\left(\mathcal{M}_{\varepsilon}-i I\right)^{-1}$ as $\varepsilon \rightarrow 0$. In other words, we study the behavior of the solutions $\mathbf{w}_{\varepsilon}, \mathbf{z}_{\varepsilon}$ of the equation

$$
\left(\mathcal{M}_{\varepsilon}-i I\right)\left(\begin{array}{l}
\mathbf{w}_{\varepsilon} \\
\mathbf{z}_{\varepsilon}
\end{array}\right)=\left(\begin{array}{l}
\mathbf{q} \\
\mathbf{r}
\end{array}\right), \quad \mathbf{q}, \mathbf{r} \in J
$$

as $\varepsilon \rightarrow 0$, and also the behavior of the corresponding strengths $\mathbf{u}_{\varepsilon}=\left(\eta^{\varepsilon}\right)^{-1} \mathbf{w}_{\varepsilon}$ and $\mathbf{v}_{\varepsilon}=\left(\mu^{\varepsilon}\right)^{-1} \mathbf{z}_{\varepsilon}$. In detail, (0.1) can be written as

$$
\begin{aligned}
\operatorname{curl}\left(\mu^{\varepsilon}\right)^{-1} \mathbf{z}_{\varepsilon}-\mathbf{w}_{\varepsilon} & =-i \mathbf{q}, \\
\operatorname{curl}\left(\eta^{\varepsilon}\right)^{-1} \mathbf{w}_{\varepsilon}+\mathbf{z}_{\varepsilon} & =i \mathbf{r}, \\
\operatorname{div} \mathbf{w}_{\varepsilon}=0, \quad \operatorname{div} \mathbf{z}_{\varepsilon} & =0 .
\end{aligned}
$$

The "homogenized" (effective) Maxwell operator $\mathcal{M}^{0}=\mathcal{M}\left(\eta^{0}, \mu^{0}\right)$ has coefficients $\eta^{0}$ and $\mu^{0}$, where $\eta^{0}$ is the effective matrix for the elliptic operator $-\operatorname{div} \eta(\mathbf{x}) \nabla$, and $\mu^{0}$ is the effective matrix for the operator $-\operatorname{div} \mu(\mathbf{x}) \nabla$. (The definition of the effective matrix is given below in (2.12).) The "elliptic" rule for the homogenized coefficients of the Maxwell system is well known. Let $\mathbf{w}_{0}, \mathbf{z}_{0}$ be the solution of the "homogenized" system

$$
\left(\mathcal{M}^{0}-i I\right)\left(\begin{array}{l}
\mathbf{w}_{0} \\
\mathbf{z}_{0}
\end{array}\right)=\left(\begin{array}{l}
\mathbf{q} \\
\mathbf{r}
\end{array}\right),
$$

and let $\mathbf{u}_{0}=\left(\eta^{0}\right)^{-1} \mathbf{w}_{0}, \mathbf{v}_{0}=\left(\mu^{0}\right)^{-1} \mathbf{z}_{0}$. It is known (see, e.g., BeLP, Sa, BaPa, ZhKO]) that the vector-valued functions $\mathbf{u}_{\varepsilon}, \mathbf{w}_{\varepsilon}, \mathbf{v}_{\varepsilon}, \mathbf{z}_{\varepsilon}$ weakly tend in $\mathfrak{G}$ to the corresponding fields $\mathbf{u}_{0}, \mathbf{w}_{0}, \mathbf{v}_{0}, \mathbf{z}_{0}$.

0.2. An application of the approach suggested in [BSu1, BSu2] allows us to obtain uniform approximations for solutions in the $\mathfrak{G}$-norm with sharp-order remainder estimates, instead of weak convergence. However, up to now we have succeeded in obtaining such approximations not for all fields. Let us explain this in detail.

It is convenient to represent each field as a sum of two terms: $\mathbf{w}_{\varepsilon}=\mathbf{w}_{\varepsilon}^{(q)}+\mathbf{w}_{\varepsilon}^{(r)}$, $\mathbf{z}_{\varepsilon}=\mathbf{z}_{\varepsilon}^{(q)}+\mathbf{z}_{\varepsilon}^{(r)}$, where the pair of vectors $\mathbf{w}_{\varepsilon}^{(q)}, \mathbf{z}_{\varepsilon}^{(q)}$ is the solution of system (0.2) with $\mathbf{r}=0$, and the pair of vectors $\mathbf{w}_{\varepsilon}^{(r)}, \mathbf{z}_{\varepsilon}^{(r)}$ is the solution of system $(0.2)$ with $\mathbf{q}=0$. Accordingly, the fields $\mathbf{u}_{\varepsilon}, \mathbf{v}_{\varepsilon}$ are also represented as sums. Thus, there are eight fields in all.

The case where $\mu=1$ was studied in Chapter 7 of [BSu2]. (However, the arguments from [BSu2, Chapter 7] can be carried over to the case of constant $\mu$ automatically.) Herewith, $\mathbf{v}_{\varepsilon}=\mathbf{z}_{\varepsilon}$, and we have six fields under consideration. For one of them, namely, for the magnetic field strength $\mathbf{v}_{\varepsilon}^{(r)}=\mathbf{z}_{\varepsilon}^{(r)}$, we have obtained the following approximation in the $\mathfrak{G}$-norm:

$$
\left\|\mathbf{v}_{\varepsilon}^{(r)}-\mathbf{v}_{0}^{(r)}\right\|_{\mathfrak{G}} \leq C \varepsilon\|\mathbf{r}\|_{\mathfrak{G}}, \quad 0<\varepsilon \leq 1
$$

Here the field $\mathbf{v}_{0}^{(r)}=\mathbf{z}_{0}^{(r)}$ (together with $\mathbf{w}_{0}^{(r)}$ ) is the solution of the homogenized system (0.3) with $\mathbf{q}=0$ (and $\mu^{0}=\mathbf{1}$ ).

The method of [BSu2, Chapter 7] consists in reducing the problem to an appropriate second order equation. The field $\mathbf{z}_{\varepsilon}^{(r)}$ (see $(0.2)$ with $\mathbf{q}=0$ and $\mu^{\varepsilon}=\mathbf{1}$ ) is the solution of the problem

$$
\operatorname{curl}\left(\eta^{\varepsilon}\right)^{-1} \operatorname{curl} \mathbf{z}_{\varepsilon}^{(r)}+\mathbf{z}_{\varepsilon}^{(r)}=i \mathbf{r}, \quad \operatorname{div} \mathbf{z}_{\varepsilon}^{(r)}=0 .
$$

Next, system (0.5) is extended, and the problem reduces to the study of the elliptic second order operator

$$
\mathcal{L}_{\varepsilon}=\mathcal{L}\left(\eta^{\varepsilon}, \mathbf{1}, 1\right)=\operatorname{curl}\left(\eta^{\varepsilon}\right)^{-1} \operatorname{curl}-\nabla \operatorname{div}
$$

acting in the space $\mathfrak{G}$ (cf. (1.3) below). This operator belongs to the general class of DO's (differential operators) distinguished and studied in BSu1, BSu2. We can apply 
the general result of $\mathrm{BSu1}, \mathrm{BSu} 2$ ] concerning approximation of the resolvent in the operator norm in $\mathfrak{G}$ in terms of the resolvent of the effective operator $\mathcal{L}^{0}$ :

$$
\left\|\left(\mathcal{L}_{\varepsilon}+I\right)^{-1}-\left(\mathcal{L}^{0}+I\right)^{-1}\right\|_{\mathfrak{G} \rightarrow \mathfrak{G}} \leq C \varepsilon, \quad 0<\varepsilon \leq 1 .
$$

Here $\mathcal{L}^{0}=\mathcal{L}\left(\eta^{0}, \mathbf{1}, 1\right)$. The approximation (0.4) follows from estimate $(0.6)$.

In [BSu2, Chapter 7], for the remaining (five) fields, only weak convergence in $\mathfrak{G}$ to the corresponding "homogenized" fields was established.

0.3. In Su1, Su2, the general case of variable periodic coefficients $\eta(\mathbf{x})$ and $\mu(\mathbf{x})$ was studied. For the four fields of eight, namely, for $\mathbf{v}_{\varepsilon}^{(r)}, \mathbf{z}_{\varepsilon}^{(r)}, \mathbf{u}_{\varepsilon}^{(q)}$, and $\mathbf{w}_{\varepsilon}^{(q)}$, uniform approximations in the $\mathfrak{G}$-norm with sharp-order remainder estimates were obtained. Besides the principal term (the solution of the homogenized system), these approximations contain terms of zero order that are rapidly oscillating as $\varepsilon \rightarrow 0$ (see relations (7.13), (7.15), (7.29), (7.31) below). These terms can be interpreted as correctors of zero order.

For the fields $\mathbf{v}_{\varepsilon}^{(r)}$ and $\mathbf{z}_{\varepsilon}^{(r)}$, the problem reduces to the study of the second order operator

$$
\mathcal{L}_{\varepsilon}=\mathcal{L}\left(\eta^{\varepsilon}, \mu^{\varepsilon}, 1\right)=\left(\mu^{\varepsilon}\right)^{-1 / 2} \operatorname{curl}\left(\eta^{\varepsilon}\right)^{-1} \operatorname{curl}\left(\mu^{\varepsilon}\right)^{-1 / 2}-\left(\mu^{\varepsilon}\right)^{1 / 2} \nabla \operatorname{div}\left(\mu^{\varepsilon}\right)^{1 / 2},
$$

and, for the fields $\mathbf{u}_{\varepsilon}^{(q)}$ and $\mathbf{w}_{\varepsilon}^{(q)}$, to the study of a similar operator $\mathcal{L}\left(\mu^{\varepsilon}, \eta^{\varepsilon}, 1\right)$. The operator (0.7) (with variable $\mu$ ) does not belong to the class of DO's distinguished in [BSu1, BSu2]. However, the results of the abstract operator-theoretic method from [BSu2, Chapter 1] can be applied to this operator. On the basis of this abstract approach, the operator $(0.7)$ was studied in [Su2], where its resolvent $\left(\mathcal{L}_{\varepsilon}+I\right)^{-1}$ was approximated in the operator norm in $\mathfrak{G}$ with order-sharp remainder estimate. This approximation is of the form

$$
\left\|\left(\mathcal{L}_{\varepsilon}+I\right)^{-1}-\left(W^{\varepsilon}\right)^{*}\left(\mathcal{L}^{0}+I\right)^{-1} W^{\varepsilon}\right\|_{\mathfrak{G} \rightarrow \mathfrak{G}} \leq C \varepsilon, \quad 0<\varepsilon \leq 1,
$$

where $\mathcal{L}^{0}=\mathcal{L}\left(\eta^{0}, \mu^{0}, 1\right)$ is the effective operator and $W^{\varepsilon}$ is some rapidly oscillating matrix-valued function. This implies the approximations mentioned above for four fields. In [Su1, Su2], for the remaining four fields, only weak convergence in $\mathfrak{G}$ to the corresponding "homogenized" fields was established.

0.4. In [BSu6], the case where the magnetic permeability $\mu$ is a constant positive matrix was considered: $\mu=\mu_{0}=$ Const. In this case, uniform approximations in the $\mathfrak{G}$-norm for all eight fields (with error estimate of order of $\varepsilon$ ) were obtained. The proof was based on a combination of the results of the papers [BSu2] and [Su1, Su2] cited above and the recent papers [BSu4, $\overline{B S u 5}$. In $\mathrm{BSu} 4, \overline{\mathrm{BSu} 5}$, in the study of the homogenization problem for the class of DO's distinguished in BSu1, BSu2, the corrector was taken into account. The problem was reduced to the study of the operator $\mathcal{L}_{\varepsilon}=\mathcal{L}\left(\eta^{\varepsilon}, \mu_{0}, 1\right)$ (cf. (0.7)), which belongs to the class of DO's distinguished in BSu1, BSu2 (if $\mu_{0}$ is constant). In [BSu6], in order to obtain the results, we used not only the principal term of approximation for the resolvent $\left(\mathcal{L}_{\varepsilon}+I\right)^{-1}$ in the operator norm in $\mathfrak{G}$ (this term can be obtained from the general theorems of [BSu2]; cf. (0.6)), but also the approximation with corrector for the resolvent in the operator norm from $\mathfrak{G}$ to $\mathfrak{G}^{1}=H^{1}\left(\mathbb{R}^{3} ; \mathbb{C}^{3}\right.$ ) (the latter approximation follows from the general results of [BSu5]).

0.5. In the present paper, we deal with the general case of variable periodic coefficients $\eta(\mathbf{x}), \mu(\mathbf{x})$. Now the complete solution of the problem is found; i.e., uniform approximations in the $\mathfrak{G}$-norm with error estimates of order of $\varepsilon$ are obtained for all physical fields. The problem reduces to the study of the operator $\mathcal{L}_{\varepsilon}=\mathcal{L}\left(\eta^{\varepsilon}, \mu^{\varepsilon}, 1\right)$ of the form (0.7). As has already been mentioned, in the general case this operator does not belong to the class of DO's distinguished in BSu1, BSu2, but the abstract part of the general results 
is applicable. The principal term of approximation for the resolvent $\left(\mathcal{L}_{\varepsilon}+I\right)^{-1}$ in the operator norm in $\mathfrak{G}$ was already obtained in [Su2] (see (0.8)). Application of this result allows us to approximate the fields $\mathbf{v}_{\varepsilon}^{(r)}$ and $\mathbf{z}_{\varepsilon}^{(r)}$ in $\mathfrak{G}$ (this was also done in [Su2]). As to the fields $\mathbf{u}_{\varepsilon}^{(r)}$ and $\mathbf{w}_{\varepsilon}^{(r)}$, they can be expressed in terms of derivatives of $\mathbf{z}_{\varepsilon}^{(r)}$ :

$$
\mathbf{w}_{\varepsilon}^{(r)}=\operatorname{curl}\left(\mu^{\varepsilon}\right)^{-1} \mathbf{z}_{\varepsilon}^{(r)}, \quad \mathbf{u}_{\varepsilon}^{(r)}=\left(\eta^{\varepsilon}\right)^{-1} \operatorname{curl}\left(\mu^{\varepsilon}\right)^{-1} \mathbf{z}_{\varepsilon}^{(r)} .
$$

Herewith, $\mathbf{u}_{\varepsilon}^{(r)}$ plays the role of the so-called "flux" for the field $\mathbf{z}_{\varepsilon}^{(r)}$. For the study of the fields $\mathbf{u}_{\varepsilon}^{(r)}$ and $\mathbf{w}_{\varepsilon}^{(r)}$, it does not suffice to use the principal term of approximation for the resolvent $\left(\mathcal{L}_{\varepsilon}+I\right)^{-1}$. We need to approximate the operator-valued function $\mathcal{L}_{\varepsilon}^{1 / 2}\left(\mathcal{L}_{\varepsilon}+I\right)^{-1}$ in the operator norm in $\mathfrak{G}$ with an error estimate of order $\varepsilon$. For this, we must take the corrector of first order into account. Such an approximation is obtained in the present paper on the basis of the abstract results of [BSu5, Chapter 1]. Next, the results for $\mathcal{L}_{\varepsilon}^{1 / 2}\left(\mathcal{L}_{\varepsilon}+I\right)^{-1}$ yield an approximation for the "flux" in the $\mathfrak{G}$-norm with an error term of order $\varepsilon$. This leads to the required approximations for the fields $\mathbf{u}_{\varepsilon}^{(r)}$ and $\mathbf{w}_{\varepsilon}^{(r)}$.

Similarly, the study of the fields $\mathbf{u}_{\varepsilon}^{(q)}, \mathbf{w}_{\varepsilon}^{(q)}, \mathbf{v}_{\varepsilon}^{(q)}, \mathbf{z}_{\varepsilon}^{(q)}$ reduces to the study of the operator $\mathcal{L}\left(\mu^{\varepsilon}, \eta^{\varepsilon}, 1\right)$ (here $\eta$ and $\mu$ interchange their roles, as compared with (0.7)). As a result, we succeed in approximating all eight fields separately. The final results are formulated for the initial four fields $\mathbf{u}_{\varepsilon}, \mathbf{w}_{\varepsilon}, \mathbf{v}_{\varepsilon}, \mathbf{z}_{\varepsilon}$. Besides the corresponding homogenized fields, approximations involve terms of zero order in $\varepsilon$ that are rapidly oscillating as $\varepsilon \rightarrow 0$. These terms play the role of correctors of zero order.

In the study of the operator $\mathcal{L}_{\varepsilon}$, we critically use the material of [Su2]. Essential difficulties are related to taking the divergence-free conditions into account. Here we use approximation for the projection onto the corresponding "divergence-free" subspace, obtained in [Su2, Theorem 5.1] (see (3.13) below).

Another difficulty is that, in the general case, we are forced to include the "smoothing" operator $\Pi_{\varepsilon}$ (see (5.4) below) in approximations (precisely, in some terms of approximations). We carefully trace the possibility to "eliminate" this smoothing operator.

Furthermore, we distinguish special cases where the form of the approximations can be simplified.

0.6. The structure of the paper. In $\S 1$, we introduce the auxiliary second order operator

$$
\mathcal{L}=\mathcal{L}(h, s, \nu)=s^{-1 / 2} \operatorname{curl} h^{-1} \operatorname{curl} s^{-1 / 2}-s^{1 / 2} \nabla \nu \operatorname{div} s^{1 / 2},
$$

which has a slightly more general form than (0.7). We discuss the expansion of the periodic operator $\mathcal{L}$ in the direct integral of the operators $\mathcal{L}(\mathbf{k})$ acting in the space $\mathfrak{H}=L_{2}\left(\Omega ; \mathbb{C}^{3}\right)$. We study the splitting of the operator $\mathcal{L}$ and the operators $\mathcal{L}(\mathbf{k})$ in the corresponding orthogonal decompositions (the Weyl decompositions). In $\S 2$, the abstract method suggested in [BSu1, BSu2] and developed in [BSu5, Chapter 1] is applied to the operator family $\mathcal{L}(\mathbf{k})$. A significant part of the material of $\S 2$ is borrowed from Su2. $\S 3$ is devoted to the approximation of the operator-valued function $\mathcal{L}(\mathbf{k})^{1 / 2}\left(\mathcal{L}(\mathbf{k})+\varepsilon^{2} I\right)^{-1}$ and its "divergence-free" part in the operator norm in $\mathfrak{H}$ with an error term of order $O(1)$. This result is based on application of the abstract Theorem 2.1 from [BSu5]. In $\S 4$, using the Gelfand transformation and the results of $\S 3$, we obtain approximations for the operator $\mathcal{L}^{1 / 2}\left(\mathcal{L}+\varepsilon^{2} I\right)^{-1}$ and its "divergence-free" part in the operator norm in $\mathfrak{G}$ with an error term $O(1)$. In $\S 5$, we study the homogenization problem for the operator $\mathcal{L}_{\varepsilon}$ with rapidly oscillating coefficients. Via the scale transformation, the results of $\S 4$ imply an approximation for the operator $\mathcal{L}_{\varepsilon}^{1 / 2}\left(\mathcal{L}_{\varepsilon}+I\right)^{-1}$ and its "divergence-free" part in the operator norm in $\mathfrak{G}$ with an error term of order $\varepsilon$. This approximation 
contains the corrector of the first order. Next, from the results for $\mathcal{L}_{\varepsilon}^{1 / 2}\left(\mathcal{L}_{\varepsilon}+I\right)^{-1}$ we deduce an approximation in the $\mathfrak{G}$-norm for the so-called "flux" (with an error term of order $\varepsilon)$. $\S 6$ is devoted to the possibility of elimination of the "smoothing operator", which must be included in approximations in the general case. The main results of the paper are contained in $\S 7$. Here we consider a stationary periodic Maxwell system. Applying theorems obtained in $\S 6$, we deduce uniform approximations in the $\mathfrak{G}$-norm for all physical fields.

0.7. Notation. Let $\mathcal{G}$ and $\mathcal{G}_{*}$ be separable Hilbert spaces. The symbols $(\cdot, \cdot)_{\mathcal{G}}$ and $\|\cdot\|_{\mathcal{G}}$ stand for the inner product and the norm in $\mathcal{G}$; the symbol $\|\cdot\|_{\mathcal{G} \rightarrow \mathcal{G}_{*}}$ denotes the norm of a continuous linear operator from $\mathcal{G}$ to $\mathcal{G}_{*}$. Sometimes we omit the indices if this does not lead to confusion. By $I=I_{\mathcal{G}}$ we denote the identity operator in $\mathcal{G}$, and by $\mathbf{0}=\mathbf{0}_{\mathcal{G}}$ the null operator. If $A: \mathcal{G} \rightarrow \mathcal{G}_{*}$ is a linear operator, then $\operatorname{Dom} A$ denotes its domain. If $\mathfrak{N}$ is a subspace in $\mathcal{G}$, then $\mathfrak{N}^{\perp}:=\mathcal{G} \ominus \mathfrak{N}$. The symbol $\langle\cdot, \cdot\rangle$ stands for the standard inner product in $\mathbb{C}^{3}$, and $|\cdot|$ is the norm of a vector in $\mathbb{C}^{3} ; \mathbf{1}$ is the unit $(3 \times 3)$-matrix. For an $(m \times n)$-matrix $a$, the symbol $|a|$ stands for the norm of this matrix viewed as a linear operator from $\mathbb{C}^{n}$ to $\mathbb{C}^{m} ; a^{*}$ stands for the Hermitian adjoint $(n \times m)$-matrix. For vectors $\mathbf{a}=\left(a^{1}, a^{2}, a^{3}\right), \mathbf{b}=\left(b^{1}, b^{2}, b^{3}\right) \in \mathbb{C}^{3}$, we use the notation $\mathbf{a} \cdot \mathbf{b}=a^{1} b^{1}+a^{2} b^{2}+a^{3} b^{3} ;$ by $\mathbf{a} \times \mathbf{b}$ we denote the vector product.

The classes $L_{p}$ of $\mathbb{C}^{n}$-valued functions in a domain $\mathcal{O} \subset \mathbb{R}^{3}$ are denoted by $L_{p}\left(\mathcal{O} ; \mathbb{C}^{n}\right)$, $1 \leq p \leq \infty$. The Sobolev classes of $\mathbb{C}^{n}$-valued functions (in a domain $\mathcal{O} \subseteq \mathbb{R}^{3}$ ) of order $s$ with integrability exponent $p$ are denoted by $W_{p}^{s}\left(\mathcal{O} ; \mathbb{C}^{n}\right)$. If $p=2$, we use the notation $H^{s}\left(\mathcal{O} ; \mathbb{C}^{n}\right), s \in \mathbb{R}$. For $n=1$ we write simply $L_{p}(\mathcal{O}), W_{p}^{s}(\mathcal{O}), H^{s}(\mathcal{O})$, etc., but (if this does not lead to confusion) sometimes we use such simplified notation also for spaces of vector-valued or matrix-valued functions.

Next, we write $\mathbf{x}=\left(x^{1}, x^{2}, x^{3}\right) \in \mathbb{R}^{3}, i D_{j}=\partial_{j}=\partial / \partial x^{j}, j=1,2,3, \nabla=\operatorname{grad}=$ $\left(\partial_{1}, \partial_{2}, \partial_{3}\right), \mathbf{D}=-i \nabla=\left(D_{1}, D_{2}, D_{3}\right)$. The notation for the vector differential operations div and curl is standard.

By $C, c, \mathcal{C}, \mathfrak{C}$ (probably, with indices and marks) we denote various constants in estimates.

0.8. Acknowledgement. The author is sincerely grateful to M. Sh. Birman for numerous useful discussions.

\section{$\S 1$. THE AUXILIARY SECOND ORDER OPERATOR}

1.1. Definition of the operator. We put

$$
\mathfrak{G}:=L_{2}\left(\mathbb{R}^{3} ; \mathbb{C}^{3}\right), \quad \mathfrak{G}_{*}:=L_{2}\left(\mathbb{R}^{3} ; \mathbb{C}^{4}\right), \quad \mathfrak{G}_{\text {loc }}:=L_{2, \text { loc }}\left(\mathbb{R}^{3} ; \mathbb{C}^{3}\right) .
$$

Let $h(\mathbf{x})$ and $s(\mathbf{x})$ be $(3 \times 3)$-matrix-valued functions in $\mathbb{R}^{3}$ with real entries and such that

$$
\begin{array}{cc}
h(\mathbf{x})>0, & h, h^{-1} \in L_{\infty}, \\
s(\mathbf{x})>0, & s, s^{-1} \in L_{\infty} .
\end{array}
$$

Let $\nu(\mathbf{x})$ be a real-valued function in $\mathbb{R}^{3}$ such that

$$
\nu(\mathbf{x})>0, \quad \nu, \nu^{-1} \in L_{\infty} .
$$

It is assumed that $h, s, \nu$ are periodic with respect to some lattice $\Gamma \subset \mathbb{R}^{3}$. By $\Omega \subset \mathbb{R}^{3}$ we denote the elementary cell of the lattice $\Gamma$. 
In the space $\mathfrak{G}$, we consider the operator $\mathcal{L}=\mathcal{L}(h, s, \nu)$ given formally by the expression

$$
\begin{aligned}
\mathcal{L} & =\mathcal{L}(h, s, \nu) \\
& =(s(\mathbf{x}))^{-1 / 2} \operatorname{curl}(h(\mathbf{x}))^{-1} \operatorname{curl}(s(\mathbf{x}))^{-1 / 2}-(s(\mathbf{x}))^{1 / 2} \nabla \nu(\mathbf{x}) \operatorname{div}(s(\mathbf{x}))^{1 / 2} .
\end{aligned}
$$

The precise definition of the operator $\mathcal{L}$ is given in terms of the quadratic form

$$
\begin{aligned}
\mathfrak{r}[\mathbf{u}, \mathbf{u}]=\int_{\mathbb{R}^{3}}\left(\left\langle h(\mathbf{x})^{-1} \operatorname{curl} s(\mathbf{x})^{-1 / 2} \mathbf{u}, \operatorname{curl} s(\mathbf{x})^{-1 / 2} \mathbf{u}\right\rangle\right. \\
\left.+\nu(\mathbf{x})\left|\operatorname{div} s(\mathbf{x})^{1 / 2} \mathbf{u}\right|^{2}\right) d \mathbf{x}, \quad \mathbf{u} \in F\left(\mathbb{R}^{3} ; s\right) .
\end{aligned}
$$

Here

$$
F\left(\mathbb{R}^{3} ; s\right):=\left\{\mathbf{u} \in \mathfrak{G}: \operatorname{div} s^{1 / 2} \mathbf{u} \in L_{2}\left(\mathbb{R}^{3}\right), \operatorname{curl} s^{-1 / 2} \mathbf{u} \in \mathfrak{G}\right\} .
$$

It is known (see, e.g., Su2, Subsections 2.1, 3.1]) that, under conditions (1.1) and (1.2), the domain (1.5) is dense in $\mathfrak{G}$, and the form (1.4) is nonnegative and closed in $\mathfrak{G}$ on the domain (1.5). By definition, the operator $\mathcal{L}$ is the selfadjoint operator in $\mathfrak{G}$ generated by the form (1.4).

Let $\mathcal{X}: \mathfrak{G} \rightarrow \mathfrak{G}_{*}$ be the operator defined by the relation

$$
\mathcal{X}=\left(\begin{array}{c}
-i h^{-1 / 2} \operatorname{curl} s^{-1 / 2} \\
-i \nu^{1 / 2} \operatorname{div} s^{1 / 2}
\end{array}\right), \quad \operatorname{Dom} \mathcal{X}=F\left(\mathbb{R}^{3} ; s\right) .
$$

Then the form (1.4) can be written as

$$
\mathfrak{r}[\mathbf{u}, \mathbf{u}]=\|\mathcal{X} \mathbf{u}\|_{\mathfrak{G}_{*}}^{2}, \quad \mathbf{u} \in F\left(\mathbb{R}^{3} ; s\right) .
$$

The operator $\mathcal{X}$ is closed together with the form l. Relation (1.6) means that the operator $\mathcal{L}$ admits a factorization: $\mathcal{L}=\mathcal{X}^{*} \mathcal{X}$.

1.2. Splitting of the operator $\mathcal{L}$. In the space $\mathfrak{G}$, we distinguish the "gradient" subspace

$$
G(s)=G\left(\mathbb{R}^{3} ; s\right)=\left\{\mathbf{u}=s^{1 / 2} \nabla \varphi: \varphi \in H_{\mathrm{loc}}^{1}\left(\mathbb{R}^{3}\right), \nabla \varphi \in \mathfrak{G}\right\} .
$$

The "divergence-free" subspace $J(s)=J\left(\mathbb{R}^{3} ; s\right)$ is defined via the orthogonal decomposition (the Weyl decomposition)

$$
\mathfrak{G}=G\left(\mathbb{R}^{3} ; s\right) \oplus J\left(\mathbb{R}^{3} ; s\right)
$$

(see $[\mathrm{Su2}$, Subsection 2.1]). Clearly, the subspace $J(s)$ consists of vector-valued functions $\mathbf{u} \in \mathfrak{G}$ such that $\operatorname{div} s^{1 / 2} \mathbf{u}=0$ (in the sense of distributions). If $s=\mathbf{1}$, we use the notation $J=J(\mathbf{1})$. By $\mathcal{P}(s)$ we denote the orthogonal projection of $\mathfrak{G}$ onto $J(s)$.

Obviously, the decomposition (1.7) reduces the operator $\mathcal{L}$. Formally, the part $\mathcal{L}_{J(s)}$ of the operator $\mathcal{L}$ acting in the "divergence-free" subspace $J(s)$ is given by the differential expression $s^{-1 / 2} \operatorname{curl} h^{-1} \operatorname{curl} s^{-1 / 2}$, and the part $\mathcal{L}_{G(s)}$ acting in the "gradient" subspace $G(s)$ corresponds to the expression $-s^{1 / 2} \nabla \nu \operatorname{div} s^{1 / 2}$. Mainly, we are interested in the operator $\mathcal{L}_{J(s)}$.

1.3. The operators $\mathcal{L}(\mathbf{k})$. We put

$$
\mathfrak{H}=L_{2}\left(\Omega ; \mathbb{C}^{3}\right), \quad \mathfrak{H}_{*}=L_{2}\left(\Omega ; \mathbb{C}^{4}\right) .
$$

In the space $\mathfrak{H}$, we consider the operator $\mathcal{L}(\mathbf{k})$ depending on the parameter $\mathbf{k} \in \mathbb{R}^{3}$ (the quasimomentum). Formally, $\mathcal{L}(\mathbf{k})$ is given by the expression

$$
\mathcal{L}(\mathbf{k})=s^{-1 / 2} \operatorname{curl}_{\mathbf{k}} h^{-1} \operatorname{curl}_{\mathbf{k}} s^{-1 / 2}-s^{1 / 2} \nabla_{\mathbf{k}} \nu \operatorname{div}_{\mathbf{k}} s^{1 / 2}
$$

with periodic boundary conditions. Here we have used the following notation:

$$
\nabla_{\mathbf{k}} \varphi:=\nabla \varphi+i \mathbf{k} \varphi, \quad \operatorname{div}_{\mathbf{k}} \mathbf{f}:=\operatorname{div} \mathbf{f}+i \mathbf{k} \cdot \mathbf{f}, \quad \operatorname{curl}_{\mathbf{k}} \mathbf{f}=\operatorname{curl} \mathbf{f}+i \mathbf{k} \times \mathbf{f} .
$$


Now, we give the precise definition of the operator $\mathcal{L}(\mathbf{k})$. We introduce the following class of vector-valued functions:

$$
F(\Omega ; s)=\left\{\mathbf{u} \in \mathfrak{H}: \operatorname{div} s^{1 / 2} \check{\mathbf{u}} \in L_{2, \operatorname{loc}}\left(\mathbb{R}^{3}\right), \operatorname{curl} s^{-1 / 2} \check{\mathbf{u}} \in \mathfrak{G}_{\text {loc }}\right\},
$$

where $\check{\mathbf{u}}$ stands for the $\Gamma$-periodic extension of a function $\mathbf{u}$ to the whole of $\mathbb{R}^{3}$. We consider the operator $\mathcal{X}(\mathbf{k}): \mathfrak{H} \rightarrow \mathfrak{H}_{*}$ given by the formula

$$
\mathcal{X}(\mathbf{k})=\left(\begin{array}{c}
-i h^{-1 / 2} \operatorname{curl}_{\mathbf{k}} s^{-1 / 2} \\
-i \nu^{1 / 2} \operatorname{div}_{\mathbf{k}} s^{1 / 2}
\end{array}\right), \quad \operatorname{Dom} \mathcal{X}(\mathbf{k})=F(\Omega ; s) .
$$

As was shown in [Su2, Subsections 2.2 and 3.2], the domain (1.8) is dense in $\mathfrak{H}$, and the operator $\mathcal{X}(\mathbf{k})$ is closed. By definition, the selfadjoint operator $\mathcal{L}(\mathbf{k})$ in the space $\mathfrak{H}$ is given by the formula

$$
\mathcal{L}(\mathbf{k})=\mathcal{X}(\mathbf{k})^{*} \mathcal{X}(\mathbf{k})
$$

In other words, $\mathcal{L}(\mathbf{k})$ is the operator in $\mathfrak{H}$ generated by the closed quadratic form

$$
\mathfrak{l}(\mathbf{k})[\mathbf{u}, \mathbf{u}]=\|\mathcal{X}(\mathbf{k}) \mathbf{u}\|_{\mathfrak{H}_{*}}^{2}, \quad \mathbf{u} \in F(\Omega ; s) .
$$

The resolvent of $\mathcal{L}(\mathbf{k})$ is compact and depends on $\mathbf{k} \in \mathbb{R}^{3}$ continuously with respect to the operator norm (see [Su2, Subsection 3.2]).

1.4. Splitting of the operators $\mathcal{L}(\mathbf{k})$. In the space $\mathfrak{H}$, we distinguish the "gradient" subspace

$$
G(s ; \mathbf{k})=G(\Omega ; s ; k)=\left\{\mathbf{u}=s^{1 / 2} \nabla_{\mathbf{k}} \varphi: \varphi \in \widetilde{H}^{1}(\Omega)\right\},
$$

depending on the parameter $\mathbf{k} \in \mathbb{R}^{3}$. Here $\widetilde{H}^{1}(\Omega)$ is the subspace in the Sobolev class $H^{1}(\Omega)$ consisting of functions whose $\Gamma$-periodic extension to $\mathbb{R}^{3}$ belongs to $H_{\text {loc }}^{1}\left(\mathbb{R}^{3}\right)$. The "divergence-free" subspace $J(s ; \mathbf{k})=J(\Omega ; s ; \mathbf{k})$ is defined as the orthogonal complement of $G(s ; \mathbf{k})$ :

$$
\mathfrak{H}=G(\Omega ; s ; \mathbf{k}) \oplus J(\Omega ; s ; \mathbf{k})
$$

(see [Su2, Subsection 2.2]). The subspace $J(s ; \mathbf{k})$ consists of vector-valued functions $\mathbf{u} \in \mathfrak{H}$ such that $\operatorname{div}_{\mathbf{k}} s^{1 / 2} \breve{\mathbf{u}}=0$ (in the sense of distributions). By $\mathcal{P}(s ; \mathbf{k})$ we denote the orthogonal projection of $\mathfrak{H}$ onto the subspace $J(s ; \mathbf{k})$.

The decomposition $(1.11)$ reduces the operator $\mathcal{L}(\mathbf{k})$. Formally, the part $(\mathcal{L}(\mathbf{k}))_{J(s ; \mathbf{k})}$ of the operator $\mathcal{L}(\mathbf{k})$ acting in the "divergence-free" subspace $J(s ; \mathbf{k})$ is given by the differential expression $s^{-1 / 2} \operatorname{curl}_{\mathbf{k}} h^{-1} \operatorname{curl}_{\mathbf{k}} s^{-1 / 2}$, and the part $(\mathcal{L}(\mathbf{k}))_{G(s ; \mathbf{k})}$ acting in the "gradient" subspace $G(s ; \mathbf{k})$ corresponds to the expression $-s^{1 / 2} \nabla_{\mathbf{k}} \nu \operatorname{div}_{\mathbf{k}} s^{1 / 2}$. (The boundary conditions are periodic.)

1.5. The Gelfand transformation. Direct integral expansion for the operator $\mathcal{L}$. Let $\widetilde{\Gamma}$ denote the lattice dual to $\Gamma$, and let $\widetilde{\Omega}$ be the central Brillouin zone of $\widetilde{\Gamma}$ (see, e.g., BSu2, Subsection 2.1.2]; $\mathrm{Su2}$, Subsection 1.2]). We introduce the Gelfand transformation $\mathcal{V}$. Initially, $\mathcal{V}$ is defined on the functions in the Schwartz class $\mathcal{S}$ by the formula

$$
\begin{array}{r}
(\mathcal{V} \mathbf{f})(\mathbf{k}, \mathbf{x})=\widetilde{\mathbf{f}}(\mathbf{k}, \mathbf{x})=|\widetilde{\Omega}|^{-1 / 2} \sum_{\mathbf{a} \in \Gamma} \exp (-i\langle\mathbf{k}, \mathbf{x}+\mathbf{a}\rangle) \mathbf{f}(\mathbf{x}+\mathbf{a}), \\
\mathbf{f} \in \mathcal{S}\left(\mathbb{R}^{3} ; \mathbb{C}^{3}\right), \quad \mathbf{x} \in \Omega, \mathbf{k} \in \widetilde{\Omega},
\end{array}
$$

and $\mathcal{V}$ extends by continuity up to a unitary mapping

$$
\mathcal{V}: L_{2}\left(\mathbb{R}^{3} ; \mathbb{C}^{3}\right) \rightarrow \int_{\widetilde{\Omega}} \oplus L_{2}\left(\Omega ; \mathbb{C}^{3}\right) d \mathbf{k}=: \mathcal{K} .
$$

Note that, under the transformation $\mathcal{V}$, the operator in $\mathfrak{G}$ of multiplication by a periodic matrix-valued function turns into multiplication by the same matrix-valued function 
on the fibers of the direct integral $\mathcal{K}$. A linear differential operator $b(\mathbf{D})$ (corresponding to some symbol $b(\boldsymbol{\xi}))$ applied to a function $\mathbf{f}$ in $\mathbb{R}^{3}$ turns into the operator $b(\mathbf{D}+\mathbf{k})$ applied to $\widetilde{\mathbf{f}}(\mathbf{k}, \cdot)$, with periodic boundary conditions.

Under the Gelfand transformation $\mathcal{V}$, the operator $\mathcal{L}$ expands in the direct integral of the operators $\mathcal{L}(\mathbf{k})$ :

$$
\mathcal{V} \mathcal{L} \mathcal{V}^{-1}=\int_{\widetilde{\Omega}} \oplus \mathcal{L}(\mathbf{k}) d \mathbf{k}
$$

In detail, (1.13) means the following. If $\mathbf{u} \in \operatorname{Dom} \mathfrak{l}=F\left(\mathbb{R}^{3} ; s\right)$, then for $\mathcal{V} \mathbf{u}=\widetilde{\mathbf{u}}$ we have

$$
\begin{aligned}
\widetilde{\mathbf{u}}(\mathbf{k}, \cdot) \in \operatorname{Dom} \mathfrak{l}(\mathbf{k}) & =F(\Omega ; s) \quad \text { for a.e. } \mathbf{k} \in \widetilde{\Omega}, \\
\mathfrak{r}[\mathbf{u}, \mathbf{u}] & =\int_{\widetilde{\Omega}} \mathfrak{r}(\mathbf{k})[\widetilde{\mathbf{u}}(\mathbf{k}, \cdot), \widetilde{\mathbf{u}}(\mathbf{k}, \cdot)] d \mathbf{k} .
\end{aligned}
$$

Conversely, if (1.14) is satisfied for $\widetilde{\mathbf{u}} \in \mathcal{K}$ and the integral on the right-hand side of (1.15) is finite, then $\mathbf{u}=\mathcal{V}^{-1} \widetilde{\mathbf{u}} \in \operatorname{Dom} \mathfrak{l}$ and (1.15) is true.

We trace the splitting of operators in the direct integral expansion. Under the Gelfand transformation, the orthogonal projection $\mathcal{P}(s)$ of $\mathfrak{G}$ onto the divergence-free subspace $J(s)$ expands in the direct integral of the orthogonal projections $\mathcal{P}(s ; \mathbf{k})$ of $\mathfrak{H}$ onto $J(s ; \mathbf{k})$ (see, e.g., [Su2, Lemma 2.13]). Hence, the operator $\mathcal{L P}(s)=\mathcal{L}_{J(s)} \oplus \mathbf{0}_{G(s)}$ (which we are interested in) expands in the direct integral of operators $\mathcal{L}(\mathbf{k}) \mathcal{P}(s ; \mathbf{k})=(\mathcal{L}(\mathbf{k}))_{J(s ; \mathbf{k})} \oplus$ $\mathbf{0}_{G(s ; \mathbf{k})}$ :

$$
\mathcal{V} \mathcal{L} \mathcal{P}(s) \mathcal{V}^{-1}=\int_{\widetilde{\Omega}} \oplus \mathcal{L}(\mathbf{k}) \mathcal{P}(s ; \mathbf{k}) d \mathbf{k} .
$$

\section{§2. Application of the general method to the operators $\mathcal{L}(\mathbf{k})$}

2.1. We apply the abstract operator-theoretic method suggested in BSu1 and BSu2, Chapter 1] and developed in [BSu3 and BSu5, Chapter 1] to the operator family $\mathcal{L}(\mathbf{k})$ defined in Subsection 1.3. Under the abstract approach, the object of study was the operator pencil of the form $A(t)=X(t)^{*} X(t)$ with $X(t)=X_{0}+t X_{1}, t \in \mathbb{R}$.

Now the parameter $\mathbf{k}$ is three-dimensional. We put (cf. [BSu2, Chapter 1])

$$
\mathbf{k}=t \boldsymbol{\theta}, \quad t=|\mathbf{k}|, \quad \boldsymbol{\theta} \in \mathbb{S}^{2},
$$

and view $t$ as the main parameter. Then all objects will depend on the additional parameter $\boldsymbol{\theta}$, and we need to make our constructions and estimates uniform with respect to $\boldsymbol{\theta}$. Introduce the notation

$$
\mathcal{L}(\mathbf{k})=: L(t, \boldsymbol{\theta}), \quad \mathcal{X}(\mathbf{k})=: X(t, \boldsymbol{\theta}) .
$$

By (1.9) and (1.10), the selfadjoint operator $L(t, \boldsymbol{\theta})$ in $\mathfrak{H}$ admits a factorization of the required form:

$$
L(t, \boldsymbol{\theta})=X(t, \boldsymbol{\theta})^{*} X(t, \boldsymbol{\theta}),
$$

where

$$
X(t, \boldsymbol{\theta})=X_{0}+t X_{1}(\boldsymbol{\theta}) .
$$

Here $X_{0}: \mathfrak{H} \rightarrow \mathfrak{H}_{*}$ is a closed operator given by the expression

$$
X_{0}=\left(\begin{array}{c}
-i h^{-1 / 2} \operatorname{curl} s^{-1 / 2} \\
-i \nu^{1 / 2} \operatorname{div} s^{1 / 2}
\end{array}\right), \quad \operatorname{Dom} X_{0}=F(\Omega ; s),
$$

and $X_{1}(\boldsymbol{\theta}): \mathfrak{H} \rightarrow \mathfrak{H}_{*}$ is a bounded operator of the form

$$
X_{1}(\boldsymbol{\theta}) \mathbf{u}=\left(\begin{array}{cc}
h^{-1 / 2} & 0 \\
0 & \nu^{1 / 2}
\end{array}\right)\left(\begin{array}{c}
\boldsymbol{\theta} \times\left(s^{-1 / 2} \mathbf{u}\right) \\
\boldsymbol{\theta} \cdot\left(s^{1 / 2} \mathbf{u}\right)
\end{array}\right), \quad \mathbf{u} \in \mathfrak{H} .
$$


As was shown in [Su2, Subsection 4.1], the operator $L(t, \boldsymbol{\theta})$ satisfies the assumptions of the general method of [BSu2, Chapter 1]. We denote

$$
\mathfrak{N}:=\operatorname{Ker} \mathcal{L}(0)=\operatorname{Ker} X_{0} .
$$

Let $P$ be the orthogonal projection of $\mathfrak{H}$ onto $\mathfrak{N}$. The subspace $\mathfrak{N}$ was described in $\mathrm{Su} 2$, (4.6)]. The elements of $\mathfrak{N}$ are parametrized by vectors $\mathbf{C} \in \mathbb{C}^{3}$. For a given $\mathbf{C} \in \mathbb{C}^{3}$, let $\Phi_{\mathbf{C}} \in \widetilde{H}^{1}(\Omega)$ be a (weak) $\Gamma$-periodic solution of the equation

$$
\operatorname{div} s(\mathbf{x})\left(\nabla \Phi_{\mathbf{C}}+\mathbf{C}\right)=0 .
$$

Then

$$
\mathfrak{N}=\left\{\mathbf{f}=s^{1 / 2}\left(\mathbf{C}+\nabla \Phi_{\mathbf{C}}\right): \mathbf{C} \in \mathbb{C}^{3}\right\} .
$$

Clearly, $\operatorname{dim} \mathfrak{N}=3$.

Thus, the number $\lambda=0$ is an eigenvalue of multiplicity 3 for the operator $\mathcal{L}(0)$. We denote by $d^{0}$ the distance from the point $\lambda=0$ to the rest of the spectrum of the operator $\mathcal{L}(0)$. We introduce the following notation (cf. [Su2, Subsections 2.1, 3.3]):

$$
\begin{aligned}
C_{0}(s) & =\max \left\{\|s\|_{L_{\infty}},\left\|s^{-1}\right\|_{L_{\infty}}\right\}, \\
C_{1} & =\max \left\{\|h\|_{L_{\infty}},\left\|\nu^{-1}\right\|_{L_{\infty}}\right\}, \\
C_{2} & =\max \left\{\left\|h^{-1}\right\|_{L_{\infty}},\|\nu\|_{L_{\infty}}\right\}, \\
c_{*}^{-1} & =C_{1} C_{0}(s) .
\end{aligned}
$$

In [Su2, (4.8)], it was shown that $d^{0}$ satisfies the estimate

$$
d^{0} \geq 4 c_{*} r_{0}^{2},
$$

where $r_{0}$ is the radius of the ball inscribed in clos $\widetilde{\Omega}$. Next, in accordance with the general method (see [BSu2, §1.1]), we fix a number $\delta \in\left(0, d^{0} / 8\right)$. It is convenient to put

$$
\delta=c_{*} r_{0}^{2} / 4 \text {. }
$$

Also, we fix a number $t^{0}$ such that

$$
t^{0} \leq \delta^{1 / 2} \min _{\boldsymbol{\theta} \in \mathbb{S}^{2}}\left\|X_{1}(\boldsymbol{\theta})\right\|^{-1} .
$$

The norm of $X_{1}(\boldsymbol{\theta})$ satisfies the estimate

$$
\left\|X_{1}(\boldsymbol{\theta})\right\|^{2} \leq 2 C_{2} C_{0}(s)
$$

(see [Su2, Subsection 4.1]). Accordingly, we put

$$
t^{0}=\delta^{1 / 2}\left(2 C_{2} C_{0}(s)\right)^{-1 / 2} .
$$

It is easily seen that $t^{0} \leq r_{0} / 2$. Then $\mathcal{B}\left(t^{0}\right) \subset \mathcal{B}\left(r_{0} / 2\right) \subset \widetilde{\Omega}$. (Here we use the notation $\mathcal{B}(r)=\left\{\mathbf{k} \in \mathbb{R}^{3}:|\mathbf{k}| \leq r\right\}$.)

Note that (see [BSu2, Chapter 1, Proposition 1.2]), for $t \in\left[0, t^{0}\right]$, the operator $L(t, \boldsymbol{\theta})$ has exactly three eigenvalues lying in the interval $[0, \delta]$, while the interval $(\delta, 3 \delta)$ is free of the spectrum of $L(t, \boldsymbol{\theta})$.

We also note that

$$
\mathcal{L}(\mathbf{k}) \geq c_{*}|\mathbf{k}|^{2} I, \quad \mathbf{k} \in \widetilde{\Omega}
$$

(see [Su2, Subsection 3.3]). 
2.2. The effective matrix. The operator of identification of kernels. Let $s^{0}$ be the constant $(3 \times 3)$-matrix defined by the relations

$$
s^{0} \mathbf{C}=|\Omega|^{-1} \int_{\Omega} s(\mathbf{x})\left(\mathbf{C}+\nabla \Phi_{\mathbf{C}}\right) d \mathbf{x}, \quad \mathbf{C} \in \mathbb{C}^{3},
$$

where $\Phi_{\mathbf{C}} \in \widetilde{H}^{1}(\Omega)$ is the solution of equation (2.1). The matrix $s^{0}$ is positive definite and has real entries. This matrix arises in the homogenization problem for the elliptic operator $-\operatorname{div} s(\mathbf{x}) \nabla$ and is called the effective matrix for this operator (see, e.g., BeLP, ZhKO, BSu2]).

If the coefficient $s(\mathbf{x})$ in the operator $\mathcal{L}$ is a constant matrix, then the kernel (2.2) consists of constant vector-valued functions and can be identified with $\mathbb{C}^{3}$. However, even in this case, it is convenient to preserve parametrization for the elements of the kernel in terms of the vector $\mathbf{C}$, as in (2.2).

In the case where $s(\mathbf{x})$ is the effective matrix $s^{0}$, we denote the kernel $\operatorname{Ker} \mathcal{L}(0)$ by $\mathfrak{N}^{0}$. We have

$$
\mathfrak{N}^{0}=\left\{\mathbf{f}^{0}=\left(s^{0}\right)^{1 / 2} \mathbf{C}: \mathbf{C} \in \mathbb{C}^{3}\right\} .
$$

Let $P_{0}$ denote the orthogonal projection of $\mathfrak{H}$ onto $\mathfrak{N}^{0}$. In other words, $P_{0}$ is the operator of averaging over the cell:

$$
P_{0} \mathbf{f}=|\Omega|^{-1} \int_{\Omega} \mathbf{f}(\mathbf{x}) d \mathbf{x} .
$$

We define the "identification of kernels" operator $\mathcal{U}: \mathfrak{N} \rightarrow \mathfrak{N}^{0}$ by the relation

$$
\mathcal{U}: \mathbf{f}=s^{1 / 2}\left(\mathbf{C}+\nabla \Phi_{\mathbf{C}}\right) \mapsto \mathbf{f}^{0}=\left(s^{0}\right)^{1 / 2} \mathbf{C}, \quad \mathbf{C} \in \mathbb{C}^{3}
$$

(see [Su2, Subsection 4.2]). As was mentioned in [Su2, Lemma 4.1], $\mathcal{U}$ is a unitary operator (this follows from the definition (2.12) of the effective matrix).

Let $\mathbf{e}_{1}, \mathbf{e}_{2}, \mathbf{e}_{3}$ be the standard orthonormal basis in $\mathbb{C}^{3}$. We put

$$
\mathbf{C}_{j}=\left(s^{0}\right)^{-1 / 2} \mathbf{e}_{j}, \quad \mathbf{g}_{j}(\mathbf{x})=s(\mathbf{x})^{1 / 2}\left(\mathbf{C}_{j}+\nabla \Phi_{\mathbf{C}_{j}}(\mathbf{x})\right), \quad j=1,2,3 .
$$

Obviously, the vectors $\mathbf{e}_{1}, \mathbf{e}_{2}, \mathbf{e}_{3}$ form an orthogonal basis in $\mathfrak{N}^{0}$, and $\left\|\mathbf{e}_{j}\right\|_{\mathfrak{H}}=|\Omega|^{1 / 2}$. Hence, the vector-valued functions $\mathbf{g}_{j}=\mathcal{U}^{-1} \mathbf{e}_{j}, j=1,2,3$, form an orthogonal basis in $\mathfrak{N}$, and $\left\|\mathbf{g}_{j}\right\|_{\mathfrak{H}}=|\Omega|^{1 / 2}$.

2.3. The operator $Z(\boldsymbol{\theta})$. Under the abstract approach (see [BSu5, Subsection 1.2]), the operator $Z: \mathfrak{H} \rightarrow \mathfrak{H}$ is defined by the following rule:

$$
Z u=0 \quad \text { for } u \in \mathfrak{N}^{\perp}, \quad Z \omega=\psi(\omega) \text { for } \omega \in \mathfrak{N},
$$

where $\psi(\omega) \in \operatorname{Dom} X_{0} \cap \mathfrak{N}^{\perp}$ is the (weak) solution of the equation

$$
X_{0}^{*}\left(X_{0} \psi(\omega)+X_{1} \omega\right)=0 .
$$

Now the operator $Z$ depends on $\boldsymbol{\theta}$ :

$$
Z(\boldsymbol{\theta}) \mathbf{u}=0 \quad \text { for } \mathbf{u} \in \mathfrak{N}^{\perp}, \quad Z(\boldsymbol{\theta}) \mathbf{f}=\mathbf{v}_{\boldsymbol{\theta}} \quad \text { for } \mathbf{f} \in \mathfrak{N} .
$$

Here $\mathbf{v}_{\boldsymbol{\theta}} \in F(\Omega ; s) \cap \mathfrak{N}^{\perp}$ is the (weak) periodic solution of the equation

$$
\begin{gathered}
s^{-1 / 2} \operatorname{curl} h^{-1}\left(\operatorname{curl} s^{-1 / 2} \mathbf{v}_{\boldsymbol{\theta}}+i \boldsymbol{\theta} \times\left(s^{-1 / 2} \mathbf{f}\right)\right) \\
-s^{1 / 2} \nabla \nu\left(\operatorname{div} s^{1 / 2} \mathbf{v}_{\boldsymbol{\theta}}+i \boldsymbol{\theta} \cdot\left(s^{1 / 2} \mathbf{f}\right)\right)=0 .
\end{gathered}
$$

Let $\mathbf{v}_{\boldsymbol{\theta}}^{(j)}$ denote the solution $\mathbf{v}_{\boldsymbol{\theta}}$ with $\mathbf{f}=\mathbf{g}_{j}$ (see (2.16)), i.e.,

$$
\mathbf{v}_{\boldsymbol{\theta}}^{(j)}=Z(\boldsymbol{\theta}) \mathbf{g}_{j}, \quad j=1,2,3 .
$$


In the standard orthonormal basis $\mathbf{e}_{1}, \mathbf{e}_{2}, \mathbf{e}_{3}$ in $\mathbb{C}^{3}$, the vector-valued functions $\mathbf{v}_{\boldsymbol{\theta}}^{(j)}(\mathbf{x})$ can be represented as columns of length 3 .

Below we shall need a description of the operator $Z(\boldsymbol{\theta}) \mathcal{U}^{*}: \mathfrak{N}^{0} \rightarrow \mathfrak{N}^{\perp}$. Let $\mathbf{f}^{0} \in \mathfrak{N}^{0}$, and let $\mathbf{f}=\mathcal{U}^{*} \mathbf{f}^{0}$. We have

$$
\mathbf{f}^{0}=\sum_{j=1}^{3}\left\langle\mathbf{f}^{0}, \mathbf{e}_{j}\right\rangle \mathbf{e}_{j}, \quad \mathbf{f}=\sum_{j=1}^{3}\left\langle\mathbf{f}^{0}, \mathbf{e}_{j}\right\rangle \mathbf{g}_{j}, \quad Z(\boldsymbol{\theta}) \mathcal{U}^{*} \mathbf{f}^{0}=Z(\boldsymbol{\theta}) \mathbf{f}=\sum_{j=1}^{3}\left\langle\mathbf{f}^{0}, \mathbf{e}_{j}\right\rangle \mathbf{v}_{\boldsymbol{\theta}}^{(j)} .
$$

We denote by $\Lambda(\mathbf{x} ; \boldsymbol{\theta})$ the $(3 \times 3)$-matrix with the columns $\mathbf{v}_{\boldsymbol{\theta}}^{(j)}(\mathbf{x}), j=1,2,3$. Then the operator $Z(\boldsymbol{\theta}) \mathcal{U}^{*}$ acts (on constant vectors $\mathbf{f}^{0} \in \mathbb{C}^{3}$ ) as multiplication by the matrix $\Lambda(\mathbf{x} ; \boldsymbol{\theta})$.

Next, let $\mathbf{v}_{l j} \in F(\Omega ; s) \cap \mathfrak{N}^{\perp}$ be the periodic solution of the problem

$$
\begin{gathered}
s^{-1 / 2} \operatorname{curl} h^{-1}\left(\operatorname{curl} s^{-1 / 2} \mathbf{v}_{l j}+i \mathbf{e}_{l} \times\left(s^{-1 / 2} \mathbf{g}_{j}\right)\right) \\
-s^{1 / 2} \nabla \nu\left(\operatorname{div} s^{1 / 2} \mathbf{v}_{l j}+i \mathbf{e}_{l} \cdot\left(s^{1 / 2} \mathbf{g}_{j}\right)\right)=0 .
\end{gathered}
$$

Let $\Lambda_{l}(\mathbf{x})$ be the $(3 \times 3)$-matrix with the columns $\mathbf{v}_{l j}(\mathbf{x}), j=1,2,3$. Clearly, we have

$$
\Lambda(\mathbf{x} ; \boldsymbol{\theta})=\sum_{l=1}^{3} \Lambda_{l}(\mathbf{x}) \theta_{l} .
$$

So, the operator $Z(\boldsymbol{\theta}) \mathcal{U}^{*}$ acts as multiplication by the matrix (2.20). Then

$$
t Z(\boldsymbol{\theta}) \mathcal{U}^{*}=\sum_{l=1}^{3}\left[\Lambda_{l}(\cdot)\right] k_{l},
$$

where $\left[\Lambda_{l}(\cdot)\right]$ is the operator of multiplication by the matrix-valued function $\Lambda_{l}(\mathbf{x})$.

2.4. The study of equation (2.19). In this subsection, we deduce some relations for the solutions of equation (2.19). We shall use these relations in what follows. Relation (2.19) implies that

$$
\operatorname{curl} h^{-1}\left(\operatorname{curl} s^{-1 / 2} \mathbf{v}_{l j}+i \mathbf{e}_{l} \times\left(s^{-1 / 2} \mathbf{g}_{j}\right)\right)=0
$$

(cf. [Su2, Subsection 4.4]). Hence,

$$
\operatorname{curl} s^{-1 / 2} \mathbf{v}_{l j}+i \mathbf{e}_{l} \times\left(s^{-1 / 2} \mathbf{g}_{j}\right)=i h(\mathbf{x})\left(\widehat{\mathbf{C}}_{l j}+\nabla \Psi_{\widehat{\mathbf{C}}_{l j}}\right),
$$

where $\widehat{\mathbf{C}}_{l j} \in \mathbb{C}^{3}$ is some constant vector and $\Psi_{\widehat{\mathbf{C}}_{l j}} \in \widetilde{H}^{1}(\Omega)$. Since $s^{-1 / 2} \mathbf{g}_{j}=\mathbf{C}_{j}+\nabla \Phi_{\mathbf{C}_{j}}$ (see (2.16)), the divergence of the left-hand side of (2.22) is equal to zero. Hence, the function $\Psi_{\widehat{\mathbf{C}}_{l j}}$ is a periodic solution of the equation

$$
\operatorname{div} h(\mathbf{x})\left(\mathbf{C}+\nabla \Psi_{\mathbf{C}}\right)=0
$$

with $\mathbf{C}=\widehat{\mathbf{C}}_{l j}$. Integrating (2.22) over $\Omega$, we obtain

$$
\mathbf{e}_{l} \times \mathbf{C}_{j}|\Omega|=\int_{\Omega} h(\mathbf{x})\left(\widehat{\mathbf{C}}_{l j}+\nabla \Psi_{\widehat{\mathbf{C}}_{l j}}\right) d \mathbf{x}=h^{0} \widehat{\mathbf{C}}_{l j}|\Omega| .
$$

Here we have used the definition of the effective matrix $h^{0}$ for the operator $-\operatorname{div} h(\mathbf{x}) \nabla$ (cf. (2.12)). Thus, by (2.16), we have

$$
\widehat{\mathbf{C}}_{l j}=\left(h^{0}\right)^{-1}\left(\mathbf{e}_{l} \times \mathbf{C}_{j}\right)=\left(h^{0}\right)^{-1}\left(\mathbf{e}_{l} \times\left(\left(s^{0}\right)^{-1 / 2} \mathbf{e}_{j}\right)\right) .
$$

It is convenient to introduce the matrix $Y_{h}(\mathbf{x})$ with the columns $\nabla \Psi_{\mathbf{e}_{j}}(\mathbf{x}), j=1,2,3$, and also the matrix $Y_{s}(\mathbf{x})$ with the columns $\nabla \Phi_{\mathbf{e}_{j}}(\mathbf{x}), j=1,2,3$. Here $\Psi_{\mathbf{e}_{j}}(\mathbf{x})$ is the 
periodic solution of equation (2.23) with $\mathbf{C}=\mathbf{e}_{j}$, and $\Phi_{\mathbf{e}_{j}}(\mathbf{x})$ is the periodic solution of equation (2.1) with $\mathbf{C}=\mathbf{e}_{j}$. Note that

$$
\begin{gathered}
s^{-1 / 2} \mathbf{g}_{j}=\mathbf{C}_{j}+\nabla \Phi_{\mathbf{C}_{j}}(\mathbf{x})=\left(\mathbf{1}+Y_{s}(\mathbf{x})\right) \mathbf{C}_{j}=\left(\mathbf{1}+Y_{s}(\mathbf{x})\right)\left(s^{0}\right)^{-1 / 2} \mathbf{e}_{j}, \quad j=1,2,3, \\
\widehat{\mathbf{C}}_{l j}+\nabla \Psi_{\widehat{\mathbf{C}}_{l j}}=\left(\mathbf{1}+Y_{h}(\mathbf{x})\right) \widehat{\mathbf{C}}_{l j}=\left(\mathbf{1}+Y_{h}(\mathbf{x})\right)\left(h^{0}\right)^{-1}\left(\mathbf{e}_{l} \times\left(\left(s^{0}\right)^{-1 / 2} \mathbf{e}_{j}\right)\right), \\
l, j=1,2,3 .
\end{gathered}
$$

2.5. The spectral germ. The crucial notion of the general method of BSu2, Chapter $1]$ is that of the spectral germ of an operator family. The germ $S(\boldsymbol{\theta})$ of the operator family $L(t, \boldsymbol{\theta})$ at $t=0$ is a selfadjoint operator acting in the 3 -dimensional space $\mathfrak{N}$. Let $\mathbf{f} \in \mathfrak{N}$, and let $\mathbf{v}_{\boldsymbol{\theta}}$ be the solution of problem (2.18). Then, by definition, the germ is the selfadjoint operator $S(\boldsymbol{\theta}): \mathfrak{N} \rightarrow \mathfrak{N}$ generated by the quadratic form

$$
(S(\boldsymbol{\theta}) \mathbf{f}, \mathbf{f})_{\mathfrak{H}}:=\left\|X_{0} \mathbf{v}_{\boldsymbol{\theta}}+X_{1}(\boldsymbol{\theta}) \mathbf{f}\right\|_{\mathfrak{H}_{*}}^{2}, \quad \mathbf{f} \in \mathfrak{N} .
$$

Concerning the spectral germ, see [BSu2, Subsection 1.6] and [Su2, Subsection 4.3].

The germ $S(\boldsymbol{\theta})$ for the operator $L(t, \boldsymbol{\theta})$ was calculated in [Su2, Subsection 4.4]. We formulate the corresponding result. Let $s^{0}$ and $h^{0}$ be the effective matrices for the elliptic operators $-\operatorname{div} s(\mathbf{x}) \nabla$ and $-\operatorname{div} h(\mathbf{x}) \nabla$, respectively. We put

$$
\underline{\nu}=\left(|\Omega|^{-1} \int_{\Omega} \nu(\mathbf{x})^{-1} d \mathbf{x}\right)^{-1}
$$

The operator

$$
\mathcal{L}^{0}=\mathcal{L}\left(h^{0}, s^{0}, \underline{\nu}\right)
$$

with constant effective coefficients is called the effective operator for $\mathcal{L}$. We denote by $L^{0}(t, \boldsymbol{\theta})=\mathcal{L}^{0}(\mathbf{k})$ the corresponding operator family. The germ $S^{0}(\boldsymbol{\theta})$ of the family $L^{0}(t, \boldsymbol{\theta})$ can easily be calculated. It acts in the space $\mathfrak{N}^{0}$ (see (2.13)) and is generated by the quadratic form

$$
\begin{array}{r}
\left(S^{0}(\boldsymbol{\theta}) \mathbf{f}^{0}, \mathbf{f}^{0}\right)_{\mathfrak{H}}=|\Omega|\left(\left\langle\left(h^{0}\right)^{-1}(\boldsymbol{\theta} \times \mathbf{C}),(\boldsymbol{\theta} \times \mathbf{C})\right\rangle+\underline{\nu}\left|\left\langle s^{0} \boldsymbol{\theta}, \mathbf{C}\right\rangle\right|^{2}\right), \\
\mathbf{f}^{0}=\left(s^{0}\right)^{1 / 2} \mathbf{C}, \quad \mathbf{C} \in \mathbb{C}^{3} .
\end{array}
$$

Then, in the standard basis $\mathbf{e}_{1}, \mathbf{e}_{2}, \mathbf{e}_{3}$ in $\mathbb{C}^{3}$, the germ $S^{0}(\boldsymbol{\theta})$ acts as multiplication by the matrix

$$
S^{0}(\boldsymbol{\theta})=\left(s^{0}\right)^{-1 / 2} r(\boldsymbol{\theta})^{*}\left(h^{0}\right)^{-1} r(\boldsymbol{\theta})\left(s^{0}\right)^{-1 / 2}+\left(s^{0}\right)^{1 / 2} \boldsymbol{\theta} \underline{\nu} \boldsymbol{\theta}^{*}\left(s^{0}\right)^{1 / 2},
$$

where $r(\boldsymbol{\theta})$ is the symbol of the operator $-i$ curl, i.e.,

$$
r(\boldsymbol{\theta})=\left(\begin{array}{ccc}
0 & -\theta_{3} & \theta_{2} \\
\theta_{3} & 0 & -\theta_{1} \\
-\theta_{2} & \theta_{1} & 0
\end{array}\right) .
$$

Note that the matrix-valued function $t^{2} S^{0}(\boldsymbol{\theta})=: S^{0}(\mathbf{k}), \mathbf{k} \in \mathbb{R}^{3}$, is none other than the symbol of the operator $\mathcal{L}^{0}$.

In [Su2, Theorem 4.2], it was shown that the spectral germ $S(\boldsymbol{\theta})$ of the family $L(t, \boldsymbol{\theta})$ is related to the germ $S^{0}(\boldsymbol{\theta})$ of the effective operator family $L^{0}(t, \boldsymbol{\theta})$ by the formula

$$
S(\boldsymbol{\theta})=\mathcal{U}^{*} S^{0}(\boldsymbol{\theta}) \mathcal{U}
$$

Here $\mathcal{U}: \mathfrak{N} \rightarrow \mathfrak{N}^{0}$ is the unitary "identification of kernels" operator introduced in (2.15).

Suppose that, from the outset, the coefficient $s(\mathbf{x})$ in the operator $\mathcal{L}$ is a constant matrix, i.e., $s(\mathbf{x})=s^{0}$. Then $\mathfrak{N}=\mathfrak{N}^{0}$ and $S(\boldsymbol{\theta})=S^{0}(\boldsymbol{\theta})$. In this case, in the terminology of [BSu2, Chapter 1], the operator families $L(t, \boldsymbol{\theta})$ and $L^{0}(t, \boldsymbol{\theta})$ are threshold equivalent. If the matrix $s(\mathbf{x})$ is variable, then the corresponding operator families are not threshold 
equivalent in the above sense. However, the germs $S(\boldsymbol{\theta})$ and $S^{0}(\boldsymbol{\theta})$ corresponding to the initial operator and the effective operator are unitarily equivalent (with a unitary operator $\mathcal{U}$ independent of $\boldsymbol{\theta})$.

The effective matrix satisfies the following known estimates: $\underline{s} \leq s^{0} \leq \bar{s}$, where

$$
\bar{s}=|\Omega|^{-1} \int_{\Omega} s(\mathbf{x}) d \mathbf{x}, \quad(\underline{s})^{-1}=|\Omega|^{-1} \int_{\Omega} s(\mathbf{x})^{-1} d \mathbf{x} .
$$

This directly implies that the norms of the matrices $s^{0}$ and $\left(s^{0}\right)^{-1}$ satisfy

$$
\left|s^{0}\right| \leq\|s\|_{L_{\infty}}, \quad\left|\left(s^{0}\right)^{-1}\right| \leq\left\|s^{-1}\right\|_{L_{\infty}} .
$$

Similar estimates are valid for $h^{0}$.

Note also that the germ $S(\boldsymbol{\theta})$ is nondegenerate and is subject to the estimate (see [Su2, Subsection 4.3])

$$
S(\boldsymbol{\theta}) \geq c_{*} I_{\mathfrak{N}}, \quad \boldsymbol{\theta} \in \mathbb{S}^{2},
$$

where $c_{*}$ is the constant defined by (2.6). The family $\mathcal{L}^{0}(\mathbf{k})$ corresponding to the effective operator (2.26) admits the same estimate from below as $\mathcal{L}(\mathbf{k})$ (see $(2.11))$ :

$$
\mathcal{L}^{0}(\mathbf{k}) \geq c_{*}|\mathbf{k}|^{2} I, \quad \mathbf{k} \in \widetilde{\Omega} .
$$

2.6. Splitting of germs. (See [Su2, Subsection 4.6].) Consider the following orthogonal decomposition of the 3 -dimensional space (2.13), depending on the parameter $\boldsymbol{\theta} \in \mathbb{S}^{2}$ :

$$
\mathfrak{N}^{0}=G_{\boldsymbol{\theta}}^{0} \oplus J_{\boldsymbol{\theta}}^{0},
$$

where

$$
\begin{array}{rlrl}
G_{\boldsymbol{\theta}}^{0} & =\left\{\mathbf{f}^{0}=\beta \mathbf{f}_{\boldsymbol{\theta}}^{0}, \mathbf{f}_{\boldsymbol{\theta}}^{0}=\left(s^{0}\right)^{1 / 2} \boldsymbol{\theta}, \beta \in \mathbb{C}\right\}, & \operatorname{dim} G_{\boldsymbol{\theta}}^{0}=1, \\
J_{\boldsymbol{\theta}}^{0}=\left\{\mathbf{f}_{\perp}^{0}=\left(s^{0}\right)^{1 / 2} \mathbf{C}_{\perp}: \mathbf{C}_{\perp} \in \mathbb{C}^{3},\left\langle s^{0} \mathbf{C}_{\perp}, \boldsymbol{\theta}\right\rangle=0\right\}, & \operatorname{dim} J_{\boldsymbol{\theta}}^{0}=2 .
\end{array}
$$

Let $P_{\boldsymbol{\theta}}^{0}$ denote the orthogonal projection of $\mathfrak{H}$ onto $J_{\boldsymbol{\theta}}^{0}$.

Obviously, the decomposition (2.30) reduces the operator $S^{0}(\boldsymbol{\theta})$. Herewith, the part $\left(S^{0}(\boldsymbol{\theta})\right)_{J_{\boldsymbol{\theta}}^{0}}$ is given by the matrix $\left(s^{0}\right)^{-1 / 2} r(\boldsymbol{\theta})^{*}\left(h^{0}\right)^{-1} r(\boldsymbol{\theta})\left(s^{0}\right)^{-1 / 2}$, and the part $\left(S^{0}(\boldsymbol{\theta})\right)_{G_{\boldsymbol{\theta}}^{0}}$ is determined by the matrix $\left(s^{0}\right)^{1 / 2} \boldsymbol{\theta} \underline{\nu} \boldsymbol{\theta}^{*}\left(s^{0}\right)^{1 / 2}$.

Under the unitary operator $\mathcal{U}^{-1}: \mathfrak{N}^{0} \rightarrow \mathfrak{N}$ (see (2.15)), the decomposition (2.30) turns into the following orthogonal decomposition of the space $(2.2)$ :

$$
\mathfrak{N}=G_{\boldsymbol{\theta}} \oplus J_{\boldsymbol{\theta}}
$$

where

$$
\begin{aligned}
G_{\boldsymbol{\theta}} & =\left\{\mathbf{f}=\beta \mathbf{f}_{\boldsymbol{\theta}}, \mathbf{f}_{\boldsymbol{\theta}}=s^{1 / 2}\left(\boldsymbol{\theta}+\nabla \Phi_{\boldsymbol{\theta}}\right), \beta \in \mathbb{C}\right\}, & \operatorname{dim} G_{\boldsymbol{\theta}}=1, \\
J_{\boldsymbol{\theta}} & =\left\{\mathbf{f}_{\perp}=s^{1 / 2}\left(\mathbf{C}_{\perp}+\nabla \Phi_{\mathbf{C}_{\perp}}\right): \mathbf{C}_{\perp} \in \mathbb{C}^{3},\left\langle s^{0} \mathbf{C}_{\perp}, \boldsymbol{\theta}\right\rangle=0\right\}, & \operatorname{dim} J_{\boldsymbol{\theta}}=2 .
\end{aligned}
$$

We denote by $P_{\boldsymbol{\theta}}$ the orthogonal projection of $\mathfrak{H}$ onto $J_{\boldsymbol{\theta}}$. Obviously, the following "intertwining" property is fulfilled:

$$
\mathcal{U} P_{\boldsymbol{\theta}}=P_{\boldsymbol{\theta}}^{0} \mathcal{U} P
$$

Recall that $P$ is the orthogonal projection of $\mathfrak{H}$ onto $\mathfrak{N}$.

The decomposition (2.31) reduces the operator $S(\boldsymbol{\theta})$. By (2.28) and (2.33), we have

$$
S(\boldsymbol{\theta}) P_{\boldsymbol{\theta}}=\mathcal{U}^{*} S^{0}(\boldsymbol{\theta}) P_{\boldsymbol{\theta}}^{0} \mathcal{U} P .
$$

It is natural to call the operator $(S(\boldsymbol{\theta}))_{J_{\boldsymbol{\theta}}}$ the spectral germ of the family $(L(t, \boldsymbol{\theta}))_{J(s ; \boldsymbol{\theta})}$, and to call $(S(\boldsymbol{\theta}))_{G_{\boldsymbol{\theta}}}$ the spectral germ of the family $(L(t, \boldsymbol{\theta}))_{G(s ; t \boldsymbol{\theta})}$ (see the discussion in $\mathrm{Su2}$, Subsection 4.6]). 


\section{$\S 3$. ApProximation OF THE OPERATOR-VALUED FUNCTION} $\mathcal{L}(\mathbf{k})^{1 / 2}\left(\mathcal{L}(\mathbf{k})+\varepsilon^{2} I\right)^{-1}$ AND OF ITS DIVERGENCE-FREE PART

3.1. Approximation of the operator $\mathcal{L}(\mathbf{k})^{1 / 2}\left(\mathcal{L}(\mathbf{k})+\varepsilon^{2} I\right)^{-1}$ for $|\mathbf{k}| \leq t^{0}$. We introduce the notation

$$
\Xi(t, \boldsymbol{\theta}, \varepsilon):=\left(t^{2} S(\boldsymbol{\theta})+\varepsilon^{2} I_{\mathfrak{N}}\right)^{-1} P .
$$

Here $P$ is the orthogonal projection of $\mathfrak{H}$ onto the subspace $\mathfrak{N}$ defined by (2.2), and $S(\boldsymbol{\theta}): \mathfrak{N} \rightarrow \mathfrak{N}$ is the spectral germ of the family $L(t, \boldsymbol{\theta})$ at $t=0$. Note that $(2.11)$ and (2.29) imply the estimates

$$
\begin{aligned}
&\left\|\left(\mathcal{L}(\mathbf{k})+\varepsilon^{2} I\right)^{-1}\right\|_{\mathfrak{H} \rightarrow \mathfrak{H}} \leq\left(c_{*} t^{2}+\varepsilon^{2}\right)^{-1}, \quad \mathbf{k} \in \widetilde{\Omega}, \quad t=|\mathbf{k}|, \\
&\|\Xi(t, \boldsymbol{\theta}, \varepsilon)\|_{\mathfrak{H} \rightarrow \mathfrak{H}} \leq\left(c_{*} t^{2}+\varepsilon^{2}\right)^{-1} .
\end{aligned}
$$

The general Theorem 1.5.5 in [BSu2] shows that for small $\varepsilon$ the operator (3.1) is the principal term of approximation for the resolvent $\left(L(t, \boldsymbol{\theta})+\varepsilon^{2} I\right)^{-1}$. Also, we have (see [Su2, Theorem 6.1])

$$
\left\|\left(L(t, \boldsymbol{\theta})+\varepsilon^{2} I\right)^{-1}-\Xi(t, \boldsymbol{\theta}, \varepsilon)\right\|_{\mathfrak{H} \rightarrow \mathfrak{H}} \leq \mathcal{C}_{1} \varepsilon^{-1}, \quad t \in\left[0, t^{0}\right], \quad 0<\varepsilon \leq 1,
$$

where the constant $\mathcal{C}_{1}$ depends on $c_{*}, \delta$, and $t^{0}$ (see (2.3)-(2.6), (2.8), and (2.10)), i.e., on the norms $\|s\|_{L_{\infty}},\left\|s^{-1}\right\|_{L_{\infty}},\|h\|_{L_{\infty}},\left\|h^{-1}\right\|_{L_{\infty}},\|\nu\|_{L_{\infty}},\left\|\nu^{-1}\right\|_{L_{\infty}}$, and on $r_{0}$. The last estimate can be rewritten as

$$
\begin{array}{r}
\left\|\left(\mathcal{L}(\mathbf{k})+\varepsilon^{2} I\right)^{-1}-(\mathcal{U} P)^{*}\left(\mathcal{L}^{0}(\mathbf{k})+\varepsilon^{2} I\right)^{-1}(\mathcal{U} P)\right\|_{\mathfrak{H} \rightarrow \mathfrak{H}} \leq \mathcal{C}_{1} \varepsilon^{-1} \\
|\mathbf{k}| \leq t^{0}, \quad 0<\varepsilon \leq 1
\end{array}
$$

(we use identity (3.9); see below). Here $\mathcal{L}^{0}(\mathbf{k})$ is the operator family in $\mathfrak{H}$ corresponding to the effective operator $\mathcal{L}^{0}=\mathcal{L}\left(h^{0}, s^{0}, \underline{\nu}\right)$, and $\mathcal{U}: \mathfrak{N} \rightarrow \mathfrak{N}^{0}$ is the unitary operator defined by (2.15). Estimate (3.4) gives the principal term of approximation for the resolvent $\left(\mathcal{L}(\mathbf{k})+\varepsilon^{2} I\right)^{-1}$ in the operator norm in $\mathfrak{H}$ with error estimate of order of $\varepsilon^{-1}$. This result was formulated in [Su2, Theorem 6.2].

Now our goal is to approximate the operator $\mathcal{L}(\mathbf{k})^{1 / 2}\left(\mathcal{L}(\mathbf{k})+\varepsilon^{2} I\right)^{-1}$ in the operator norm in $\mathfrak{H}$ with an error term of order $O(1)$. For this, it is necessary to incorporate the corrector in approximation. The corresponding general results were obtained in BSu5, Chapter 1]. Applying [BSu5, Theorem 2.1], we directly obtain the following estimate:

$$
\begin{array}{r}
\left\|L(t, \boldsymbol{\theta})^{1 / 2}\left(\left(L(t, \boldsymbol{\theta})+\varepsilon^{2} I\right)^{-1}-(I+t Z(\boldsymbol{\theta})) \Xi(t, \boldsymbol{\theta}, \varepsilon)\right)\right\|_{\mathfrak{H} \rightarrow \mathfrak{H}} \leq \mathcal{C}_{2}, \\
0<\varepsilon \leq 1, \quad t \in\left[0, t^{0}\right] .
\end{array}
$$

Here $\Xi(t, \boldsymbol{\theta}, \varepsilon)$ is the operator defined by $(3.1), Z(\boldsymbol{\theta})$ is the operator defined by (2.17), and $t^{0}$ is the number defined by (2.10). The constant $\mathcal{C}_{2}$ depends only on $\|s\|_{L_{\infty}},\left\|s^{-1}\right\|_{L_{\infty}}$, $\|h\|_{L_{\infty}},\left\|h^{-1}\right\|_{L_{\infty}},\|\nu\|_{L_{\infty}},\left\|\nu^{-1}\right\|_{L_{\infty}}$, and on $r_{0}$. The role of the corrector in (3.5) is played by the term $t Z(\boldsymbol{\theta}) \Xi(t, \boldsymbol{\theta}, \varepsilon)$, which is added to the principal term of approximation, i.e., to $\Xi(t, \boldsymbol{\theta}, \varepsilon)$.

We calculate the operator $(I+t Z(\boldsymbol{\theta})) \Xi(t, \boldsymbol{\theta}, \varepsilon)$ involved in (3.5). First, we use (2.28) to transform the operator (3.1):

$$
\Xi(t, \boldsymbol{\theta}, \varepsilon)=\left(t^{2} \mathcal{U}^{*} S^{0}(\boldsymbol{\theta}) \mathcal{U}+\varepsilon^{2} \mathcal{U}^{*} \mathcal{U}\right)^{-1} P=\mathcal{U}^{*} \Xi^{0}(t, \boldsymbol{\theta}, \varepsilon) \mathcal{U} P,
$$

where

$$
\Xi^{0}(t, \boldsymbol{\theta}, \varepsilon)=\left(t^{2} S^{0}(\boldsymbol{\theta})+\varepsilon^{2} I_{\mathfrak{N}^{0}}\right)^{-1} P_{0} .
$$

Obviously, the presence of the projection $P_{0}$ allows us to write

$$
t^{2} S^{0}(\boldsymbol{\theta}) P_{0}=S^{0}(\mathbf{k}) P_{0}=S^{0}(\mathbf{D}+\mathbf{k}) P_{0}=\mathcal{L}^{0}(\mathbf{k}) P_{0}
$$


(see (2.14) and (2.27)). Then for the operator (3.7) we obtain

$$
\Xi^{0}(t, \boldsymbol{\theta}, \varepsilon)=\left(\mathcal{L}^{0}(\mathbf{k})+\varepsilon^{2} I\right)^{-1} P_{0} .
$$

As a result, (3.6) and (3.8) imply that

$$
\Xi(t, \boldsymbol{\theta}, \varepsilon)=\mathcal{U}^{*}\left(\mathcal{L}^{0}(\mathbf{k})+\varepsilon^{2} I\right)^{-1} P_{0} \mathcal{U} P=(\mathcal{U} P)^{*}\left(\mathcal{L}^{0}(\mathbf{k})+\varepsilon^{2} I\right)^{-1} P_{0}(\mathcal{U} P) .
$$

Now we calculate the operator $t Z(\boldsymbol{\theta}) \Xi(t, \boldsymbol{\theta}, \varepsilon)$, using (3.9) and (2.21). We have

$$
\begin{aligned}
t Z(\boldsymbol{\theta}) \Xi(t, \boldsymbol{\theta}, \varepsilon) & =t Z(\boldsymbol{\theta}) \mathcal{U}^{*}\left(\mathcal{L}^{0}(\mathbf{k})+\varepsilon^{2} I\right)^{-1} P_{0}(\mathcal{U P}) \\
& =\sum_{l=1}^{3}\left[\Lambda_{l}\right] k_{l}\left(\mathcal{L}^{0}(\mathbf{k})+\varepsilon^{2} I\right)^{-1} P_{0}(\mathcal{U} P) \\
& =\sum_{l=1}^{3}\left[\Lambda_{l}\right]\left(D_{l}+k_{l}\right)\left(\mathcal{L}^{0}(\mathbf{k})+\varepsilon^{2} I\right)^{-1} P_{0}(\mathcal{U} P) .
\end{aligned}
$$

Once again, using the presence of the projection $P_{0}$, here we have replaced $k_{l}$ by $\left(D_{l}+k_{l}\right)$.

Now, (3.5), (3.9), and (3.10) imply the following result.

Theorem 3.1. Let $\mathcal{L}=\mathcal{L}(h, s, \nu)$ be the operator in $\mathfrak{G}$ defined in Subsection 1.1, and let $\mathcal{L}(\mathbf{k})$ be the corresponding operator family in $\mathfrak{H}$ defined in Subsection 1.3. Denote by $\mathcal{L}^{0}=\mathcal{L}\left(h^{0}, s^{0}, \underline{\nu}\right)$ the effective operator, and by $\mathcal{L}^{0}(\mathbf{k})$ the corresponding operator family in $\mathfrak{H}$. Consider the orthogonal projection $P$ of $\mathfrak{H}$ onto the subspace $\mathfrak{N}$ defined by (2.2), and let $P_{0}$ be the orthogonal projection of $\mathfrak{H}$ onto $\mathfrak{N}^{0}$ defined by (2.14). Let $\mathcal{U}: \mathfrak{N} \rightarrow \mathfrak{N}^{0}$ be the unitary operator defined by (2.15). Denote by $\left[\Lambda_{l}\right], l=1,2,3$, the operator of multiplication by the matrix-valued function $\Lambda_{l}(\mathbf{x})$ with the columns $\mathbf{v}_{l j}(\mathbf{x}), j=1,2,3$, where $\mathbf{v}_{l j} \in F(\Omega ; s) \cap \mathfrak{N}^{\perp}$ is the periodic solution of problem (2.19). Finally, let $t^{0}$ be the number defined by (2.10). Then, for $|\mathbf{k}| \leq t^{0}$ and $0<\varepsilon \leq 1$, we have

$$
\begin{aligned}
\| \mathcal{L}(\mathbf{k})^{1 / 2}( & \left(\mathcal{L}(\mathbf{k})+\varepsilon^{2} I\right)^{-1}-(\mathcal{U} P)^{*}\left(\mathcal{L}^{0}(\mathbf{k})+\varepsilon^{2} I\right)^{-1} P_{0}(\mathcal{U} P) \\
& \left.\quad-\sum_{l=1}^{3}\left[\Lambda_{l}\right]\left(D_{l}+k_{l}\right)\left(\mathcal{L}^{0}(\mathbf{k})+\varepsilon^{2} I\right)^{-1} P_{0}(\mathcal{U} P)\right) \|_{\mathfrak{H} \rightarrow \mathfrak{H}} \leq \mathcal{C}_{2} .
\end{aligned}
$$

The constant $\mathcal{C}_{2}$ depends only on $\|s\|_{L_{\infty}},\left\|s^{-1}\right\|_{L_{\infty}},\|h\|_{L_{\infty}},\left\|h^{-1}\right\|_{L_{\infty}},\|\nu\|_{L_{\infty}},\left\|\nu^{-1}\right\|_{L_{\infty}}$, and on $r_{0}$.

3.2. Approximation of the operator $\mathcal{L}(\mathbf{k})^{1 / 2}\left(\mathcal{L}(\mathbf{k})+\varepsilon^{2} I\right)^{-1} \mathcal{P}(s ; \mathbf{k})$ for $|\mathbf{k}| \leq t^{0}$. Recall that $\mathcal{P}(s ; \mathbf{k})$ denotes the orthogonal projection of $\mathfrak{H}$ onto the "divergence-free" subspace $J(s ; \mathbf{k})$ defined in Subsection 1.4. We multiply the operators under the normsign in (3.5) by $\mathcal{P}(s ; \mathbf{k})=\mathcal{P}(s ; t \boldsymbol{\theta})$ from the right. Then (3.5) implies the estimate

$$
\begin{aligned}
& \left\|L(t, \boldsymbol{\theta})^{1 / 2}\left(\left(L(t, \boldsymbol{\theta})+\varepsilon^{2} I\right)^{-1} \mathcal{P}(s ; t \boldsymbol{\theta})-(I+t Z(\boldsymbol{\theta})) \Xi(t, \boldsymbol{\theta}, \varepsilon) P \mathcal{P}(s ; t \boldsymbol{\theta})\right)\right\|_{\mathfrak{H} \rightarrow \mathfrak{H}} \\
& \quad \leq \mathcal{C}_{2}, \quad 0<\varepsilon \leq 1, \quad t \in\left[0, t^{0}\right] .
\end{aligned}
$$

Theorem 5.1 in Su2] yields

$$
\left\|P \mathcal{P}(s ; t \boldsymbol{\theta})-P_{\boldsymbol{\theta}}\right\|_{\mathfrak{H} \rightarrow \mathfrak{H}} \leq C_{3} t, \quad 0<t \leq r_{0}, \quad \boldsymbol{\theta} \in \mathbb{S}^{2} .
$$

Recall that $P_{\boldsymbol{\theta}}$ is the orthogonal projection of $\mathfrak{H}$ onto the two-dimensional subspace $J_{\boldsymbol{\theta}}$ defined by (2.32). The constant $C_{3}$ in (3.13) depends only on $\|s\|_{L_{\infty}}$ and $\left\|s^{-1}\right\|_{L_{\infty}}$, and on $r_{0}$.

We consider the operator

$$
\Sigma(t, \boldsymbol{\theta}, \varepsilon):=L(t, \boldsymbol{\theta})^{1 / 2}(I+t Z(\boldsymbol{\theta})) \Xi(t, \boldsymbol{\theta}, \varepsilon)\left(P \mathcal{P}(s ; t \boldsymbol{\theta})-P_{\boldsymbol{\theta}}\right)
$$


and show that its norm does not exceed some constant. The operator (3.14) is represented as

$$
\Sigma(t, \boldsymbol{\theta}, \varepsilon)=\Sigma_{1}(t, \boldsymbol{\theta}, \varepsilon)+\Sigma_{2}(t, \boldsymbol{\theta}, \varepsilon),
$$

where

$$
\begin{aligned}
& \Sigma_{1}(t, \boldsymbol{\theta}, \varepsilon)=\left(L(t, \boldsymbol{\theta})^{1 / 2} P\right) \Xi(t, \boldsymbol{\theta}, \varepsilon)\left(P \mathcal{P}(s ; t \boldsymbol{\theta})-P_{\boldsymbol{\theta}}\right), \\
& \Sigma_{2}(t, \boldsymbol{\theta}, \varepsilon)=t\left(L(t, \boldsymbol{\theta})^{1 / 2} Z(\boldsymbol{\theta})\right) \Xi(t, \boldsymbol{\theta}, \varepsilon)\left(P \mathcal{P}(s ; t \boldsymbol{\theta})-P_{\boldsymbol{\theta}}\right) .
\end{aligned}
$$

We estimate the norm of the operator $L(t, \boldsymbol{\theta})^{1 / 2} P$. Since $X_{0} P=0$, by $(2.9)$ we have

$$
\left\|L(t, \boldsymbol{\theta})^{1 / 2} P \mathbf{u}\right\|_{\mathfrak{H}}=\left\|\left(X_{0}+t X_{1}(\boldsymbol{\theta})\right) P \mathbf{u}\right\|_{\mathfrak{H}_{*}}=t\left\|X_{1}(\boldsymbol{\theta}) P \mathbf{u}\right\|_{\mathfrak{H}_{*}} \leq\left(2 C_{2} C_{0}(s)\right)^{1 / 2} t\|\mathbf{u}\|_{\mathfrak{H}} .
$$

Hence,

$$
\left\|L(t, \boldsymbol{\theta})^{1 / 2} P\right\|_{\mathfrak{H} \rightarrow \mathfrak{H}} \leq\left(2 C_{2} C_{0}(s)\right)^{1 / 2} t, \quad \mathbf{k}=t \boldsymbol{\theta} \in \widetilde{\Omega} .
$$

Relations (3.3), (3.13), and (3.18) imply the following estimate for the operator (3.16):

$$
\left\|\Sigma_{1}(t, \boldsymbol{\theta}, \varepsilon)\right\|_{\mathfrak{H} \rightarrow \mathfrak{H}} \leq\left(2 C_{2} C_{0}(s)\right)^{1 / 2} C_{3} c_{*}^{-1}, \quad t \in\left[0, r_{0}\right] .
$$

In order to estimate the operator (3.17), we need the following lemma.

Lemma 3.2. Let $Z(\boldsymbol{\theta})$ be the operator defined by (2.17). Then

$$
\left\|L(t, \boldsymbol{\theta})^{1 / 2} Z(\boldsymbol{\theta})\right\|_{\mathfrak{H} \rightarrow \mathfrak{H}} \leq C_{4}, \quad \mathbf{k}=t \boldsymbol{\theta} \in \widetilde{\Omega} .
$$

Here

$$
C_{4}=\left(2 C_{2} C_{0}(s)\right)^{1 / 2}+C_{2} C_{0}(s) c_{*}^{-1 / 2} r_{1} r_{0}^{-1},
$$

and $r_{1}=\max \{|\mathbf{k}|: \mathbf{k} \in \operatorname{clos} \widetilde{\Omega}\}$.

Proof. Since $Z(\boldsymbol{\theta}) \mathbf{u}=0$ for $\mathbf{u} \in \mathfrak{N}^{\perp}$, it suffices to estimate the norm $\left\|L(t, \boldsymbol{\theta})^{1 / 2} Z(\boldsymbol{\theta}) \mathbf{f}\right\|_{\mathfrak{H}}$ for $\mathbf{f} \in \mathfrak{N}$. We have $Z(\boldsymbol{\theta}) \mathbf{f}=\mathbf{v}_{\boldsymbol{\theta}}$, where $\mathbf{v}_{\boldsymbol{\theta}} \in F(\Omega ; s) \cap \mathfrak{N}^{\perp}$ is the solution of equation (2.18), i.e., the equation

$$
X_{0}^{*}\left(X_{0} \mathbf{v}_{\boldsymbol{\theta}}+X_{1}(\boldsymbol{\theta}) \mathbf{f}\right)=0 .
$$

Then

$$
\left\|L(t, \boldsymbol{\theta})^{1 / 2} Z(\boldsymbol{\theta}) \mathbf{f}\right\|_{\mathfrak{H}}=\left\|\left(X_{0}+t X_{1}(\boldsymbol{\theta})\right) \mathbf{v}_{\boldsymbol{\theta}}\right\|_{\mathfrak{H}_{*}} \leq\left\|X_{0} \mathbf{v}_{\boldsymbol{\theta}}\right\|_{\mathfrak{H}_{*}}+t\left\|X_{1}(\boldsymbol{\theta})\right\|\left\|\mathbf{v}_{\boldsymbol{\theta}}\right\|_{\mathfrak{H}}
$$

Equation (3.21) implies

$$
\left\|X_{0} \mathbf{v}_{\boldsymbol{\theta}}\right\|_{\mathfrak{H}_{*}} \leq\left\|X_{1}(\boldsymbol{\theta}) \mathbf{f}\right\|_{\mathfrak{H}_{*}} .
$$

Next, since $\mathbf{v}_{\boldsymbol{\theta}} \in \operatorname{Dom} X_{0} \cap \mathfrak{N}^{\perp}$ and $d^{0}$ is the distance from the point $\lambda=0$ to the rest of the spectrum of the operator $\mathcal{L}(0)=X_{0}^{*} X_{0}$, we have

$$
\left\|\mathbf{v}_{\boldsymbol{\theta}}\right\|_{\mathfrak{H}^{2}}^{2} \leq\left(d^{0}\right)^{-1}\left\|X_{0} \mathbf{v}_{\boldsymbol{\theta}}\right\|_{\mathfrak{H}_{*}}^{2} .
$$

Consequently, by (3.23),

$$
\left\|\mathbf{v}_{\boldsymbol{\theta}}\right\|_{\mathfrak{H}} \leq\left(d^{0}\right)^{-1 / 2}\left\|X_{1}(\boldsymbol{\theta}) \mathbf{f}\right\|_{\mathfrak{H}_{*}}
$$

Finally, from (3.22)-(3.24) we deduce that

$$
\left\|L(t, \boldsymbol{\theta})^{1 / 2} Z(\boldsymbol{\theta}) \mathbf{f}\right\|_{\mathfrak{H}} \leq\left(1+\left(d^{0}\right)^{-1 / 2} t\left\|X_{1}(\boldsymbol{\theta})\right\|\right)\left\|X_{1}(\boldsymbol{\theta}) \mathbf{f}\right\|_{\mathfrak{H}_{*}} .
$$

Using estimates (2.7), (2.9), and the restriction $t \leq r_{1}$, we arrive at inequality (3.20). 
Now, from (3.3), (3.13), and (3.20) we obtain the following estimate for the norm of the operator (3.17):

$$
\left\|\Sigma_{2}(t, \boldsymbol{\theta}, \varepsilon)\right\|_{\mathfrak{H} \rightarrow \mathfrak{H}} \leq C_{3} C_{4} c_{*}^{-1}, \quad t \in\left[0, r_{0}\right] .
$$

Relations (3.15), (3.19), and (3.25) imply an estimate for the norm of the operator (3.14):

$$
\|\Sigma(t, \boldsymbol{\theta}, \varepsilon)\|_{\mathfrak{H} \rightarrow \mathfrak{H}} \leq C_{3} c_{*}^{-1}\left(C_{4}+\left(2 C_{2} C_{0}(s)\right)^{1 / 2}\right), \quad t \in\left[0, r_{0}\right] .
$$

As a result, from (3.12), (3.14), and (3.26) we deduce that

$$
\begin{gathered}
\left\|L(t, \boldsymbol{\theta})^{1 / 2}\left(\left(L(t, \boldsymbol{\theta})+\varepsilon^{2} I\right)^{-1} \mathcal{P}(s ; t \boldsymbol{\theta})-(I+t Z(\boldsymbol{\theta})) \Xi(t, \boldsymbol{\theta}, \varepsilon) P_{\boldsymbol{\theta}}\right)\right\|_{\mathfrak{H} \rightarrow \mathfrak{H}} \leq \mathcal{C}_{2}^{\prime}, \\
0<\varepsilon \leq 1, \quad t \in\left[0, t^{0}\right],
\end{gathered}
$$

where

$$
\mathcal{C}_{2}^{\prime}=\mathcal{C}_{2}+C_{3} c_{*}^{-1}\left(C_{4}+\left(2 C_{2} C_{0}(s)\right)^{1 / 2}\right)
$$

We transform the operator $(I+t Z(\boldsymbol{\theta})) \Xi(t, \boldsymbol{\theta}, \varepsilon) P_{\boldsymbol{\theta}}$ occurring in (3.27). By (3.6) and (2.33), we have

$$
\Xi(t, \boldsymbol{\theta}, \varepsilon) P_{\boldsymbol{\theta}}=\mathcal{U}^{*} \Xi^{0}(t, \boldsymbol{\theta}, \varepsilon) \mathcal{U} P_{\boldsymbol{\theta}}=\mathcal{U}^{*} \Xi^{0}(t, \boldsymbol{\theta}, \varepsilon) P_{\boldsymbol{\theta}}^{0}(\mathcal{U} P) .
$$

Also,

$$
P_{\boldsymbol{\theta}}^{0}=\mathcal{P}\left(s^{0} ; \mathbf{k}\right) P_{0}=P_{0} \mathcal{P}\left(s^{0} ; \mathbf{k}\right) P_{0}
$$

(see [Su2, (5.2)]). Relations (3.8) and (3.29) show that

$$
\Xi^{0}(t, \boldsymbol{\theta}, \varepsilon) P_{\boldsymbol{\theta}}^{0}=\left(\mathcal{L}^{0}(\mathbf{k})+\varepsilon^{2} I\right)^{-1} \mathcal{P}\left(s^{0} ; \mathbf{k}\right) P_{0} .
$$

As a result, $(3.28),(3.30)$, and $(2.21)$ lead to the identities:

$$
\begin{gathered}
\Xi(t, \boldsymbol{\theta}, \varepsilon) P_{\boldsymbol{\theta}}=(\mathcal{U} P)^{*}\left(\mathcal{L}^{0}(\mathbf{k})+\varepsilon^{2} I\right)^{-1} \mathcal{P}\left(s^{0} ; \mathbf{k}\right) P_{0}(\mathcal{U} P), \\
t Z(\boldsymbol{\theta}) \Xi(t, \boldsymbol{\theta}, \varepsilon) P_{\boldsymbol{\theta}}=\sum_{l=1}^{3}\left[\Lambda_{l}\right]\left(D_{l}+k_{l}\right)\left(\mathcal{L}^{0}(\mathbf{k})+\varepsilon^{2} I\right)^{-1} \mathcal{P}\left(s^{0} ; \mathbf{k}\right) P_{0}(\mathcal{U} P) .
\end{gathered}
$$

Combining this with (3.27), we arrive at the following theorem.

Theorem 3.3. Suppose that the conditions of Theorem 3.1 are satisfied. Let $\mathcal{P}(s ; \mathbf{k})$ be the orthogonal projection of $\mathfrak{H}$ onto the subspace $J(s ; \mathbf{k})$ defined in Subsection 1.4 , and let $\mathcal{P}\left(s^{0} ; \mathbf{k}\right)$ be the orthogonal projection onto the corresponding subspace $J\left(s^{0} ; \mathbf{k}\right)$. Then, for $|\mathbf{k}| \leq t^{0}$ and $0<\varepsilon \leq 1$, we have

$$
\begin{aligned}
\| \mathcal{L}(\mathbf{k})^{1 / 2}( & \left(\mathcal{L}(\mathbf{k})+\varepsilon^{2} I\right)^{-1} \mathcal{P}(s ; \mathbf{k})-(\mathcal{U} P)^{*}\left(\mathcal{L}^{0}(\mathbf{k})+\varepsilon^{2} I\right)^{-1} \mathcal{P}\left(s^{0} ; \mathbf{k}\right) P_{0}(\mathcal{U} P) \\
& \left.-\sum_{l=1}^{3}\left[\Lambda_{l}\right]\left(D_{l}+k_{l}\right)\left(\mathcal{L}^{0}(\mathbf{k})+\varepsilon^{2} I\right)^{-1} \mathcal{P}\left(s^{0} ; \mathbf{k}\right) P_{0}(\mathcal{U} P)\right) \|_{\mathfrak{H} \rightarrow \mathfrak{H}} \leq \mathcal{C}_{2}^{\prime} .
\end{aligned}
$$

The constant $\mathcal{C}_{2}^{\prime}$ depends only on $\|s\|_{L_{\infty}},\left\|s^{-1}\right\|_{L_{\infty}},\|h\|_{L_{\infty}},\left\|h^{-1}\right\|_{L_{\infty}},\|\nu\|_{L_{\infty}},\left\|\nu^{-1}\right\|_{L_{\infty}}$, and the parameters of the lattice $\Gamma$.

3.3. Estimates for $|\mathbf{k}|>t^{0}$. Estimates for $\mathbf{k} \in \widetilde{\Omega} \backslash \mathcal{B}\left(t^{0}\right)$ are rougher than those for $|\mathbf{k}| \leq t^{0}$, because each term under the norm sign in (3.11) (and in (3.31)) is estimated separately. First, by (3.2), we have

$$
\left\|\mathcal{L}(\mathbf{k})^{1 / 2}\left(\mathcal{L}(\mathbf{k})+\varepsilon^{2} I\right)^{-1}\right\|_{\mathfrak{H} \rightarrow \mathfrak{H}} \leq c_{*}^{-1 / 2}\left(t^{0}\right)^{-1}, \quad \mathbf{k} \in \widetilde{\Omega} \backslash \mathcal{B}\left(t^{0}\right) .
$$

Using (3.9), (3.18), and (3.3), we obtain

$$
\begin{aligned}
& \left\|\mathcal{L}(\mathbf{k})^{1 / 2}(\mathcal{U} P)^{*}\left(\mathcal{L}^{0}(\mathbf{k})+\varepsilon^{2} I\right)^{-1} P_{0}(\mathcal{U} P)\right\|_{\mathfrak{H} \rightarrow \mathfrak{H}} \\
& \quad=\left\|\left(L(t, \boldsymbol{\theta})^{1 / 2} P\right) \Xi(t, \boldsymbol{\theta}, \varepsilon)\right\|_{\mathfrak{H} \rightarrow \mathfrak{H}} \leq\left(2 C_{2} C_{0}(s)\right)^{1 / 2} t\left(c_{*} t^{2}+\varepsilon^{2}\right)^{-1} \\
& \quad \leq\left(2 C_{2} C_{0}(s)\right)^{1 / 2} c_{*}^{-1}\left(t^{0}\right)^{-1}, \quad \mathbf{k} \in \widetilde{\Omega} \backslash \mathcal{B}\left(t^{0}\right) .
\end{aligned}
$$


From (3.10), (3.3), and (3.20), it follows that

$$
\begin{aligned}
& \left\|\sum_{l=1}^{3} \quad \mathcal{L}(\mathbf{k})^{1 / 2}\left[\Lambda_{l}\right]\left(D_{l}+k_{l}\right)\left(\mathcal{L}^{0}(\mathbf{k})+\varepsilon^{2} I\right)^{-1} P_{0}(\mathcal{U} P)\right\|_{\mathfrak{H} \rightarrow \mathfrak{H}} \\
& \quad=t\left\|L(t, \boldsymbol{\theta})^{1 / 2} Z(\boldsymbol{\theta}) \Xi(t, \boldsymbol{\theta}, \varepsilon)\right\|_{\mathfrak{H} \rightarrow \mathfrak{H}} \\
& \quad \leq C_{4} t\left(c_{*} t^{2}+\varepsilon^{2}\right)^{-1} \leq C_{4} c_{*}^{-1}\left(t^{0}\right)^{-1}, \quad \mathbf{k} \in \widetilde{\Omega} \backslash \mathcal{B}\left(t^{0}\right) .
\end{aligned}
$$

As a result, using (3.32)-(3.34), we see that the following estimate for the operator in (3.11) for $\mathbf{k} \in \widetilde{\Omega} \backslash \mathcal{B}\left(t^{0}\right)$ is valid:

$$
\begin{aligned}
\| \mathcal{L}(\mathbf{k})^{1 / 2}( & \left(\mathcal{L}(\mathbf{k})+\varepsilon^{2} I\right)^{-1}-(\mathcal{U} P)^{*}\left(\mathcal{L}^{0}(\mathbf{k})+\varepsilon^{2} I\right)^{-1} P_{0}(\mathcal{U} P) \\
& \left.-\sum_{l=1}^{3}\left[\Lambda_{l}\right]\left(D_{l}+k_{l}\right)\left(\mathcal{L}^{0}(\mathbf{k})+\varepsilon^{2} I\right)^{-1} P_{0}(\mathcal{U} P)\right) \|_{\mathfrak{H} \rightarrow \mathfrak{H}} \leq \mathcal{C}_{3}, \\
& \mathbf{k} \in \widetilde{\Omega} \backslash \mathcal{B}\left(t^{0}\right),
\end{aligned}
$$

where

$$
\mathcal{C}_{3}:=c_{*}^{-1 / 2}\left(t^{0}\right)^{-1}+\left(\left(2 C_{2} C_{0}(s)\right)^{1 / 2}+C_{4}\right) c_{*}^{-1}\left(t^{0}\right)^{-1} .
$$

Similarly, we can estimate the operator occurring in (3.31) for $\mathbf{k} \in \widetilde{\Omega} \backslash \mathcal{B}\left(t^{0}\right)$; its norm is dominated by the same constant $\mathcal{C}_{3}$.

Now Theorems 3.1 and 3.3, together with estimate (3.35) and its analog for the operator in (3.31), imply the following result.

Theorem 3.4. Let $\mathcal{L}=\mathcal{L}(h, s, \nu)$ be the operator in $\mathfrak{G}$ defined in Subsection 1.1, and let $\mathcal{L}(\mathbf{k})$ be the corresponding family of operators in $\mathfrak{H}$ defined in Subsection 1.3. Denote by $\mathcal{L}^{0}=\mathcal{L}\left(h^{0}, s^{0}, \underline{\nu}\right)$ the effective operator, and by $\mathcal{L}^{0}(\mathbf{k})$ the corresponding operator family in $\mathfrak{H}$. Let $P$ be the orthogonal projection of $\mathfrak{H}$ onto the subspace $\mathfrak{N}$ defined by (2.2), and let $P_{0}$ be the orthogonal projection of $\mathfrak{H}$ onto $\mathfrak{N}^{0}$ defined by (2.14). Consider the unitary operator $\mathcal{U}: \mathfrak{N} \rightarrow \mathfrak{N}^{0}$ defined by (2.15). Finally, let $\left[\Lambda_{l}\right], l=1,2,3$, be the operator of multiplication by the matrix-valued function $\Lambda_{l}(\mathbf{x})$ with the columns $\mathbf{v}_{l j}(\mathbf{x}), j=1,2,3$, where $\mathbf{v}_{l j} \in F(\Omega ; s) \cap \mathfrak{N}^{\perp}$ is the periodic solution of problem (2.19).

$1^{\circ}$. Under the above conditions, for $0<\varepsilon \leq 1$ we have

$$
\begin{aligned}
\| \mathcal{L}(\mathbf{k})^{1 / 2}( & \left(\mathcal{L}(\mathbf{k})+\varepsilon^{2} I\right)^{-1}-(\mathcal{U} P)^{*}\left(\mathcal{L}^{0}(\mathbf{k})+\varepsilon^{2} I\right)^{-1} P_{0}(\mathcal{U} P) \\
& \left.\quad-\sum_{l=1}^{3}\left[\Lambda_{l}\right]\left(D_{l}+k_{l}\right)\left(\mathcal{L}^{0}(\mathbf{k})+\varepsilon^{2} I\right)^{-1} P_{0}(\mathcal{U} P)\right) \|_{\mathfrak{H} \rightarrow \mathfrak{H}} \leq \mathcal{C}_{4}, \quad \mathbf{k} \in \widetilde{\Omega},
\end{aligned}
$$

where

$$
\mathcal{C}_{4}=\max \left\{\mathcal{C}_{2}, \mathcal{C}_{3}\right\}
$$

$2^{\circ}$. Let $\mathcal{P}(s ; \mathbf{k})$ be the orthogonal projection of $\mathfrak{H}$ onto the subspace $J(s ; \mathbf{k})$ defined in Subsection 1.4 , and let $\mathcal{P}\left(s^{0} ; \mathbf{k}\right)$ be the orthogonal projection onto the corresponding subspace $J\left(s^{0} ; \mathbf{k}\right)$. Then, under the above conditions, for $0<\varepsilon \leq 1$ we have

$$
\begin{aligned}
\| \mathcal{L}(\mathbf{k})^{1 / 2}( & \left(\mathcal{L}(\mathbf{k})+\varepsilon^{2} I\right)^{-1} \mathcal{P}(s ; \mathbf{k})-(\mathcal{U} P)^{*}\left(\mathcal{L}^{0}(\mathbf{k})+\varepsilon^{2} I\right)^{-1} \mathcal{P}\left(s^{0} ; \mathbf{k}\right) P_{0}(\mathcal{U} P) \\
& \left.-\sum_{l=1}^{3}\left[\Lambda_{l}\right]\left(D_{l}+k_{l}\right)\left(\mathcal{L}^{0}(\mathbf{k})+\varepsilon^{2} I\right)^{-1} \mathcal{P}\left(s^{0} ; \mathbf{k}\right) P_{0}(\mathcal{U} P)\right) \|_{\mathfrak{H} \rightarrow \mathfrak{H}} \leq \mathcal{C}_{4}^{\prime}, \quad \mathbf{k} \in \widetilde{\Omega},
\end{aligned}
$$

where

$$
\mathcal{C}_{4}^{\prime}=\max \left\{\mathcal{C}_{2}^{\prime}, \mathcal{C}_{3}\right\}
$$


The constants $\mathcal{C}_{4}$ and $\mathcal{C}_{4}^{\prime}$ depend only on $\|s\|_{L_{\infty}},\left\|s^{-1}\right\|_{L_{\infty}},\|h\|_{L_{\infty}},\left\|h^{-1}\right\|_{L_{\infty}},\|\nu\|_{L_{\infty}}$, $\left\|\nu^{-1}\right\|_{L_{\infty}}$, and the parameters of the lattice $\Gamma$.

\section{§4. Approximation of the operator $\mathcal{L}^{1 / 2}\left(\mathcal{L}+\varepsilon^{2} I\right)^{-1}$ AND OF ITS DIVERGENCE-FREE PART}

4.1. We return to the study of the operator $\mathcal{L}$ acting in the space $\mathfrak{G}=L_{2}\left(\mathbb{R}^{3} ; \mathbb{C}^{3}\right)$ and defined in Subsection 1.1. The principal term of approximation for the resolvent $\left(\mathcal{L}+\varepsilon^{2} I\right)^{-1}$ was obtained in $\underline{\mathrm{Su} 2}$, Theorem 8.6] (on the basis of estimate (3.4)):

$$
\left\|\left(\mathcal{L}+\varepsilon^{2} I\right)^{-1}-W^{*}\left(\mathcal{L}^{0}+\varepsilon^{2} I\right)^{-1} W\right\|_{\mathfrak{G} \rightarrow \mathfrak{G}} \leq \mathcal{C}_{5} \varepsilon^{-1}, \quad 0<\varepsilon \leq 1 .
$$

Here $W^{*}$ is the operator of multiplication by the matrix $W(\mathbf{x})^{*}$ with the columns $\mathbf{g}_{j}(\mathbf{x})$, $j=1,2,3$ (see (2.16)). The constant $\mathcal{C}_{5}$ depends only on $\|s\|_{L_{\infty}},\left\|s^{-1}\right\|_{L_{\infty}},\|h\|_{L_{\infty}}$, $\left\|h^{-1}\right\|_{L_{\infty}},\|\nu\|_{L_{\infty}},\left\|\nu^{-1}\right\|_{L_{\infty}}$, and the parameters of the lattice $\Gamma$.

In [Su2, Theorem 8.7], approximation for the "divergence-free" part of the resolvent $\left(\mathcal{L}+\varepsilon^{2} I\right)^{-1}$ was also obtained:

$$
\left\|\left(\mathcal{L}+\varepsilon^{2} I\right)^{-1} \mathcal{P}(s)-W^{*}\left(\mathcal{L}^{0}+\varepsilon^{2} I\right)^{-1} \mathcal{P}\left(s^{0}\right) W\right\|_{\mathfrak{G} \rightarrow \mathfrak{G}} \leq \mathcal{C}_{6} \varepsilon^{-1}, \quad 0<\varepsilon \leq 1 .
$$

Here $\mathcal{P}(s)$ is the orthogonal projection of $\mathfrak{G}$ onto the "divergence-free" subspace $J(s)$ defined in Subsection 1.2, and $\mathcal{P}\left(s^{0}\right)$ is the orthogonal projection onto the corresponding subspace $J\left(s^{0}\right)$. The constant $\mathcal{C}_{6}$ in (4.2) depends only on $\|s\|_{L_{\infty}},\left\|s^{-1}\right\|_{L_{\infty}},\|h\|_{L_{\infty}}$, $\left\|h^{-1}\right\|_{L_{\infty}}$, and the parameters of the lattice $\Gamma$.

4.2. Now our goal is to approximate the operator $\mathcal{L}^{1 / 2}\left(\mathcal{L}+\varepsilon^{2} I\right)^{-1}$ and its "divergencefree" part in the operator norm in $\mathfrak{G}$ with an error estimate of order of $O(1)$. The corresponding approximations are deduced from Theorem 3.4 with the help of the Gelfand transformation (see Subsection 1.5). By (1.13), we have

$$
\mathcal{L}^{1 / 2}\left(\mathcal{L}+\varepsilon^{2} I\right)^{-1}=\mathcal{V}^{*}\left(\int_{\widetilde{\Omega}} \oplus \mathcal{L}(\mathbf{k})^{1 / 2}\left(\mathcal{L}(\mathbf{k})+\varepsilon^{2} I\right)^{-1} d \mathbf{k}\right) \mathcal{V}
$$

Similarly, the operator $\mathcal{L}^{1 / 2}$ expands in the direct integral of the operators $\mathcal{L}(\mathbf{k})^{1 / 2}$, the resolvent $\left(\mathcal{L}^{0}+\varepsilon^{2} I\right)^{-1}$ expands in the direct integral of the operators $\left(\mathcal{L}^{0}(\mathbf{k})+\varepsilon^{2} I\right)^{-1}$, and $D_{l}\left(\mathcal{L}^{0}+\varepsilon^{2} I\right)^{-1}$ expands in the direct integral of the operators $\left(D_{l}+k_{l}\right)\left(\mathcal{L}^{0}(\mathbf{k})+\varepsilon^{2} I\right)^{-1}$.

Next, it is easily seen (see [Su2, Subsection 7.2]) that

$$
\Pi:=\mathcal{V}^{*}\left(\int_{\tilde{\Omega}} \oplus P_{0} d \mathbf{k}\right) \mathcal{V}=\mathcal{V}^{*}\left[P_{0}\right] \mathcal{V}
$$

is a pseudodifferential operator $(\Psi D O)$ in $\mathfrak{G}$ with the symbol $\chi_{\widetilde{\Omega}}(\boldsymbol{\xi})$, where $\chi_{\widetilde{\Omega}}(\boldsymbol{\xi})$ is the indicator of the set $\widetilde{\Omega}$, i.e.,

$$
(\Pi \mathbf{f})(\mathbf{x})=(2 \pi)^{-3 / 2} \int_{\widetilde{\Omega}} e^{i\langle\mathbf{x}, \boldsymbol{\xi}\rangle} \widehat{\mathbf{f}}(\boldsymbol{\xi}) d \boldsymbol{\xi} .
$$

Here $\widehat{\mathbf{f}}$ is the Fourier image of a function $\mathbf{f}$. Note that the $\Psi D O \Pi$ is an orthogonal projection, and it commutes with any $\Psi D O$ with symbol independent of $\mathbf{x}$.

By Proposition 7.5 in [Su2], the operator

$$
\mathcal{W}^{*}:=\mathcal{V}^{*}\left(\int_{\widetilde{\Omega}} \oplus(\mathcal{U} P)^{*} d \mathbf{k}\right) \mathcal{V}=\mathcal{V}^{*}\left[(\mathcal{U} P)^{*}\right] \mathcal{V}
$$

can be written as

$$
\mathcal{W}^{*}=W^{*} \Pi,
$$

where $\Pi$ is the $\Psi \mathrm{DO}(4.3)$ and $W^{*}$ is the operator of multiplication by the matrix $W(\mathbf{x})^{*}$ with the columns $\mathbf{g}_{j}(\mathbf{x}), j=1,2,3$ (this operator was already introduced in Subsection 4.1). 
Finally, we take into account the fact that, under the Gelfand transformation, the operator $\left[\Lambda_{l}\right]$ in $\mathfrak{G}$ of multiplication by the periodic matrix-valued function $\Lambda_{l}(\mathbf{x})$ turns into the operator of multiplication by the same matrix-valued function on the fibers of the direct integral $\mathcal{K}$ (see (1.12)).

The following theorem can be deduced from Theorem 3.4(1 $\left.{ }^{\circ}\right)$ by using the Gelfand transformation.

Theorem 4.1. Let $\mathcal{L}=\mathcal{L}(h, s, \nu)$ be the operator in $\mathfrak{G}$ defined in Subsection 1.1. Denote by $\mathcal{L}^{0}=\mathcal{L}\left(h^{0}, s^{0}, \underline{\nu}\right)$ the effective operator, and by $\Pi$ the $\Psi D O(4.3)$. Let $W^{*}$ be the operator of multiplication by the matrix $W(\mathbf{x})^{*}$ with the columns $\mathbf{g}_{j}(\mathbf{x}), j=1,2,3$, defined by (2.16). Next, let $\left[\Lambda_{l}\right], l=1,2,3$, be the operator of multiplication by the matrix-valued function $\Lambda_{l}(\mathbf{x})$ with the columns $\mathbf{v}_{l j}(\mathbf{x}), j=1,2,3$, where $\mathbf{v}_{l j} \in F(\Omega ; s) \cap \mathfrak{N}^{\perp}$ is the periodic solution of problem (2.19). Then for $0<\varepsilon \leq 1$ we have

$$
\left\|\mathcal{L}^{1 / 2}\left(\left(\mathcal{L}+\varepsilon^{2} I\right)^{-1}-W^{*}\left(\mathcal{L}^{0}+\varepsilon^{2} I\right)^{-1} \Pi W-\sum_{l=1}^{3}\left[\Lambda_{l}\right] D_{l}\left(\mathcal{L}^{0}+\varepsilon^{2} I\right)^{-1} \Pi W\right)\right\|_{\mathfrak{G} \rightarrow \mathfrak{G}} \leq \mathcal{C}_{4} .
$$

The constant $\mathcal{C}_{4}$ depends only on $\|s\|_{L_{\infty}},\left\|s^{-1}\right\|_{L_{\infty}},\|h\|_{L_{\infty}},\left\|h^{-1}\right\|_{L_{\infty}},\|\nu\|_{L_{\infty}},\left\|\nu^{-1}\right\|_{L_{\infty}}$, and the parameters of the lattice $\Gamma$.

Recall that, under the Gelfand transformation, the projection $\mathcal{P}(s)$ expands in the direct intergal of the projections $\mathcal{P}(s ; \mathbf{k})$ (see discussion at the end of Subsection 1.5). Then, by the Gelfand transformation, Theorem 3.4 $\left(2^{\circ}\right)$ implies the following result.

Theorem 4.2. Suppose that the conditions of Theorem 4.1 are satisfied. Let $\mathcal{P}(s)$ be the orthogonal projection of $\mathfrak{G}$ onto the subspace $J(s)$ defined in Subsection 1.2 , and let $\mathcal{P}\left(s^{0}\right)$ be the orthogonal projection onto the subspace $J\left(s^{0}\right)$. Then for $0<\varepsilon \leq 1$ we have

$$
\begin{aligned}
\| \mathcal{L}^{1 / 2}\left(\left(\mathcal{L}+\varepsilon^{2} I\right)^{-1}\right. & \mathcal{P}(s)-W^{*}\left(\mathcal{L}^{0}+\varepsilon^{2} I\right)^{-1} \mathcal{P}\left(s^{0}\right) \Pi W \\
& \left.-\sum_{l=1}^{3}\left[\Lambda_{l}\right] D_{l}\left(\mathcal{L}^{0}+\varepsilon^{2} I\right)^{-1} \mathcal{P}\left(s^{0}\right) \Pi W\right) \|_{\mathfrak{G} \rightarrow \mathfrak{G}} \leq \mathcal{C}_{4}^{\prime} .
\end{aligned}
$$

The constant $\mathcal{C}_{4}^{\prime}$ depends only on $\|s\|_{L_{\infty}},\left\|s^{-1}\right\|_{L_{\infty}},\|h\|_{L_{\infty}},\left\|h^{-1}\right\|_{L_{\infty}},\|\nu\|_{L_{\infty}},\left\|\nu^{-1}\right\|_{L_{\infty}}$, and the parameters of the lattice $\Gamma$.

\section{$\S 5$. Homogenization Problem for the operator $\mathcal{L}$}

5.1. Below, if $\varphi(\mathbf{x})$ is a measurable $\Gamma$-periodic function, we systematically use the notation $\varphi^{\varepsilon}(\mathbf{x}):=\varphi\left(\varepsilon^{-1} \mathbf{x}\right)$. For the operator $\mathcal{L}=\mathcal{L}(h, s, \nu)$ defined in Subsection 1.1, we consider the family of operators

$$
\mathcal{L}_{\varepsilon}=\mathcal{L}\left(h^{\varepsilon}, s^{\varepsilon}, \nu^{\varepsilon}\right), \quad \varepsilon>0 .
$$

The coefficients of the operator (5.1) oscillate rapidly as $\varepsilon \rightarrow 0$. The homogenization problem consists in an investigation of the behavior of the resolvent $\left(\mathcal{L}_{\varepsilon}+I\right)^{-1}$ and its "divergence-free" part (i.e., the operator $\left.\left(\mathcal{L}_{\varepsilon}+I\right)^{-1} \mathcal{P}\left(s^{\varepsilon}\right)\right)$, as $\varepsilon \rightarrow 0$.

In [Su2, Theorem 9.1], the principal term of approximation for the resolvent was found:

$$
\left\|\left(\mathcal{L}_{\varepsilon}+I\right)^{-1}-\left(W^{\varepsilon}\right)^{*}\left(\mathcal{L}^{0}+I\right)^{-1} W^{\varepsilon}\right\|_{\mathfrak{G} \rightarrow \mathfrak{G}} \leq \mathcal{C}_{5} \varepsilon, \quad 0<\varepsilon \leq 1 .
$$

Here $\mathcal{L}^{0}=\mathcal{L}\left(h^{0}, s^{0}, \underline{\nu}\right)$ is the effective operator, $W$ is the matrix-valued function introduced in Subsection 4.1, and $W^{\varepsilon}(\mathbf{x})=W\left(\varepsilon^{-1} \mathbf{x}\right)$. Estimate (5.2) is deduced from (4.1) by a scale transformation. We see that the principal term of approximation is given by the resolvent of the effective operator $\mathcal{L}^{0}$ sandwiched with rapidly oscillating factors. In [Su2, Theorem 9.2], the "divergence-free" part of the resolvent was also approximated:

$$
\left\|\left(\mathcal{L}_{\varepsilon}+I\right)^{-1} \mathcal{P}\left(s^{\varepsilon}\right)-\left(W^{\varepsilon}\right)^{*}\left(\mathcal{L}^{0}+I\right)^{-1} \mathcal{P}\left(s^{0}\right) W^{\varepsilon}\right\|_{\mathfrak{G} \rightarrow \mathfrak{G}} \leq \mathcal{C}_{6} \varepsilon, \quad 0<\varepsilon \leq 1 .
$$


This estimate follows from (4.2) by a scale transformation.

5.2. Now we want to approximate the operator $\mathcal{L}_{\varepsilon}^{1 / 2}\left(\mathcal{L}_{\varepsilon}+I\right)^{-1}$ in the operator norm in $\mathfrak{G}$ with an error estimate of order $\varepsilon$. For this, besides the principal term of approximation, we must take the term $\varepsilon K(\varepsilon)$ into account, where $K(\varepsilon)$ is the so-called corrector.

Let $T_{\varepsilon}$ denote the unitary scale transformation in $\mathfrak{G}$ :

$$
\left(T_{\varepsilon} \mathbf{u}\right)(\mathbf{y})=\varepsilon^{3 / 2} \mathbf{u}(\varepsilon \mathbf{y}) .
$$

The following relations are checked easily:

$$
\begin{gathered}
\mathcal{L}_{\varepsilon}=\varepsilon^{-2} T_{\varepsilon}^{*} \mathcal{L} T_{\varepsilon} \\
\left(\mathcal{L}_{\varepsilon}+I\right)^{-1}=\varepsilon^{2} T_{\varepsilon}^{*}\left(\mathcal{L}+\varepsilon^{2} I\right)^{-1} T_{\varepsilon}, \quad\left(\mathcal{L}^{0}+I\right)^{-1}=\varepsilon^{2} T_{\varepsilon}^{*}\left(\mathcal{L}^{0}+\varepsilon^{2} I\right)^{-1} T_{\varepsilon} \\
D_{l}\left(\mathcal{L}^{0}+I\right)^{-1}=\varepsilon T_{\varepsilon}^{*} D_{l}\left(\mathcal{L}^{0}+\varepsilon^{2} I\right)^{-1} T_{\varepsilon} \\
{\left[\Lambda_{l}^{\varepsilon}\right]=T_{\varepsilon}^{*}\left[\Lambda_{l}\right] T_{\varepsilon}, \quad\left[W^{\varepsilon}\right]=T_{\varepsilon}^{*}[W] T_{\varepsilon} .}
\end{gathered}
$$

We put

$$
\Pi_{\varepsilon}:=T_{\varepsilon}^{*} \Pi T_{\varepsilon} .
$$

Then $\Pi_{\varepsilon}$ is a $\Psi D O$ in $\mathfrak{G}$ with the symbol $\chi_{\widetilde{\Omega} / \varepsilon}(\boldsymbol{\xi})$, i.e.,

$$
\left(\Pi_{\varepsilon} \mathbf{f}\right)(\mathbf{x})=(2 \pi)^{-3 / 2} \int_{\widetilde{\Omega} / \varepsilon} e^{i\langle\mathbf{x}, \boldsymbol{\xi}\rangle} \widehat{\mathbf{f}}(\boldsymbol{\xi}) d \boldsymbol{\xi} .
$$

The operator (5.4) plays the role of the "smoothing operator" in approximations. Note that $\Pi_{\varepsilon}$ is the orthogonal projection in $\mathfrak{G}$, and it commutes with all pseudodifferential operators with symbols independent of $\mathbf{x}$.

The following result is deduced from Theorem 4.1 with the help of a scale transformation.

Theorem 5.1. Let $\mathcal{L}_{\varepsilon}$ be the operator in $\mathfrak{G}$ defined by (5.1). Denote by $\mathcal{L}^{0}=\mathcal{L}\left(h^{0}, s^{0}, \underline{\nu}\right)$ the effective operator, and by $\Pi_{\varepsilon}$ the $\Psi D O(5.4)$. Let $W(\mathbf{x})^{*}$ be the matrix with the columns $\mathbf{g}_{j}(\mathbf{x}), j=1,2,3$, defined by (2.16). Let $W^{\varepsilon}$ be the operator of multiplication by the matrix $W^{\varepsilon}(\mathbf{x})=W\left(\varepsilon^{-1} \mathbf{x}\right)$. Next, let $\Lambda_{l}(\mathbf{x})$ be the matrix with the columns $\mathbf{v}_{l j}(\mathbf{x})$, $j=1,2,3$, where $\mathbf{v}_{l j} \in F(\Omega ; s) \cap \mathfrak{N}^{\perp}$ is the periodic solution of problem (2.19), and let $\left[\Lambda_{l}^{\varepsilon}\right], l=1,2,3$, be the operator of multiplication by the matrix-valued function $\Lambda_{l}^{\varepsilon}(\mathbf{x})=$ $\Lambda_{l}\left(\varepsilon^{-1} \mathbf{x}\right)$. Then for $0<\varepsilon \leq 1$ we have

$$
\begin{aligned}
\| \mathcal{L}_{\varepsilon}^{1 / 2}\left(\left(\mathcal{L}_{\varepsilon}+I\right)^{-1}\right. & -\left(W^{\varepsilon}\right)^{*}\left(\mathcal{L}^{0}+I\right)^{-1} \Pi_{\varepsilon} W^{\varepsilon} \\
& \left.-\varepsilon \sum_{l=1}^{3}\left[\Lambda_{l}^{\varepsilon}\right] D_{l}\left(\mathcal{L}^{0}+I\right)^{-1} \Pi_{\varepsilon} W^{\varepsilon}\right) \|_{\mathfrak{G} \rightarrow \mathfrak{G}} \leq \mathcal{C}_{4} \varepsilon .
\end{aligned}
$$

The constant $\mathcal{C}_{4}$ depends only on $\|s\|_{L_{\infty}},\left\|s^{-1}\right\|_{L_{\infty}},\|h\|_{L_{\infty}},\left\|h^{-1}\right\|_{L_{\infty}},\|\nu\|_{L_{\infty}},\left\|\nu^{-1}\right\|_{L_{\infty}}$, and the parameters of the lattice $\Gamma$.

The role of the corrector in (5.5) is played by the operator

$$
K(\varepsilon)=\sum_{l=1}^{3}\left[\Lambda_{l}^{\varepsilon}\right] D_{l}\left(\mathcal{L}^{0}+I\right)^{-1} \Pi_{\varepsilon} W^{\varepsilon} .
$$

The corrector (5.6) involves the bordering rapidly oscillating factors $\Lambda_{l}^{\varepsilon}, W^{\varepsilon}$, and also the smoothing operator $\Pi_{\varepsilon}$.

The identities (see [Su2, (9.7)])

$$
\mathcal{P}\left(s^{\varepsilon}\right)=T_{\varepsilon}^{*} \mathcal{P}(s) T_{\varepsilon}, \quad \mathcal{P}\left(s^{0}\right)=T_{\varepsilon}^{*} \mathcal{P}\left(s^{0}\right) T_{\varepsilon}
$$

and a scale transformation allow us to deduce the next statement from Theorem 4.2. 
Theorem 5.2. Suppose that the conditions of Theorem 5.1 are satisfied. Let $\mathcal{P}\left(s^{\varepsilon}\right)$ be the orthogonal projection of $\mathfrak{G}$ onto the subspace $J\left(s^{\varepsilon}\right)$, and let $\mathcal{P}\left(s^{0}\right)$ be the orthogonal projection onto $J\left(s^{0}\right)$. Then for $0<\varepsilon \leq 1$ we have

$$
\begin{aligned}
\| \mathcal{L}_{\varepsilon}^{1 / 2}\left(\left(\mathcal{L}_{\varepsilon}+I\right)^{-1} \mathcal{P}\left(s^{\varepsilon}\right)\right. & -\left(W^{\varepsilon}\right)^{*}\left(\mathcal{L}^{0}+I\right)^{-1} \mathcal{P}\left(s^{0}\right) \Pi_{\varepsilon} W^{\varepsilon} \\
& \left.-\varepsilon \sum_{l=1}^{3}\left[\Lambda_{l}^{\varepsilon}\right] D_{l}\left(\mathcal{L}^{0}+I\right)^{-1} \mathcal{P}\left(s^{0}\right) \Pi_{\varepsilon} W^{\varepsilon}\right) \|_{\mathfrak{G} \rightarrow \mathfrak{G}} \leq \mathcal{C}_{4}^{\prime} \varepsilon .
\end{aligned}
$$

The constant $\mathcal{C}_{4}^{\prime}$ depends only on $\|s\|_{L_{\infty}},\left\|s^{-1}\right\|_{L_{\infty}},\|h\|_{L_{\infty}},\left\|h^{-1}\right\|_{L_{\infty}},\|\nu\|_{L_{\infty}},\left\|\nu^{-1}\right\|_{L_{\infty}}$, and the parameters of the lattice $\Gamma$.

5.3. Approximation of fluxes. For applications to the Maxwell system, we need to approximate the so-called "fluxes" in the $\mathfrak{G}$-norm. First, we formulate the result of Theorem 5.2 in terms of solutions. Let $\mathbf{f}_{\varepsilon}$ be the solution of the problem

$$
\left(s^{\varepsilon}\right)^{-1 / 2} \operatorname{curl}\left(h^{\varepsilon}\right)^{-1} \operatorname{curl}\left(s^{\varepsilon}\right)^{-1 / 2} \mathbf{f}_{\varepsilon}+\mathbf{f}_{\varepsilon}=\left(s^{\varepsilon}\right)^{-1 / 2} \mathbf{F}, \quad \operatorname{div}\left(s^{\varepsilon}\right)^{1 / 2} \mathbf{f}_{\varepsilon}=0, \quad \mathbf{F} \in J .
$$

Here $J=J(\mathbf{1})$ is the subspace in $\mathfrak{G}$ distinguished by the condition div $\mathbf{u}=0$. Automatically, the right-hand side of (5.8) satisfies $\left(s^{\varepsilon}\right)^{-1 / 2} \mathbf{F} \in J\left(s^{\varepsilon}\right)$. Then

$$
\mathbf{f}_{\varepsilon}=\left(\mathcal{L}_{\varepsilon}+I\right)^{-1} \mathcal{P}\left(s^{\varepsilon}\right)\left(\left(s^{\varepsilon}\right)^{-1 / 2} \mathbf{F}\right) .
$$

Let $\mathbf{f}_{\varepsilon}^{0}$ denote the solution of the problem

$$
\left(s^{0}\right)^{-1 / 2} \operatorname{curl}\left(h^{0}\right)^{-1} \operatorname{curl}\left(s^{0}\right)^{-1 / 2} \mathbf{f}_{\varepsilon}^{0}+\mathbf{f}_{\varepsilon}^{0}=\mathcal{P}\left(s^{0}\right) W^{\varepsilon}\left(s^{\varepsilon}\right)^{-1 / 2} \mathbf{F}, \quad \operatorname{div}\left(s^{0}\right)^{1 / 2} \mathbf{f}_{\varepsilon}^{0}=0 .
$$

Then

$$
\mathbf{f}_{\varepsilon}^{0}=\left(\mathcal{L}^{0}+I\right)^{-1} \mathcal{P}\left(s^{0}\right) W^{\varepsilon}\left(s^{\varepsilon}\right)^{-1 / 2} \mathbf{F} .
$$

Estimate (5.7) means that

$$
\left\|\mathcal{L}_{\varepsilon}^{1 / 2}\left(\mathbf{f}_{\varepsilon}-\left(W^{\varepsilon}\right)^{*} \Pi_{\varepsilon} \mathbf{f}_{\varepsilon}^{0}-\varepsilon \sum_{l=1}^{3} \Lambda_{l}^{\varepsilon} D_{l} \Pi_{\varepsilon} \mathbf{f}_{\varepsilon}^{0}\right)\right\|_{\mathfrak{G}} \leq \mathcal{C}_{4}^{\prime} \varepsilon\left\|s^{-1}\right\|_{L_{\infty}}^{1 / 2}\|\mathbf{F}\|_{\mathfrak{G}}, \quad 0<\varepsilon \leq 1 .
$$

The role of the "flux" for the solution $\mathbf{f}_{\varepsilon}$ of equation (5.8) is played by the vector-valued function

$$
\mathbf{p}_{\varepsilon}:=\left(h^{\varepsilon}\right)^{-1} \operatorname{curl}\left(s^{\varepsilon}\right)^{-1 / 2} \mathbf{f}_{\varepsilon} .
$$

We consider an approximation of the vector-valued function (5.11) and also of the function $\operatorname{curl}\left(s^{\varepsilon}\right)^{-1 / 2} \mathbf{f}_{\varepsilon}$ in the $\mathfrak{G}$-norm with an error estimate of order $\varepsilon$. Since

$$
\begin{array}{r}
\left\|\mathcal{L}_{\varepsilon}^{1 / 2} \mathbf{v}\right\|_{\mathfrak{G}}^{2}=\left\|\left(h^{\varepsilon}\right)^{-1 / 2} \operatorname{curl}\left(s^{\varepsilon}\right)^{-1 / 2} \mathbf{v}\right\|_{\mathfrak{G}}^{2}+\left\|\left(\nu^{\varepsilon}\right)^{1 / 2} \operatorname{div}\left(s^{\varepsilon}\right)^{1 / 2} \mathbf{v}\right\|_{L_{2}\left(\mathbb{R}^{3}\right)}^{2}, \\
\mathbf{v} \in F\left(\mathbb{R}^{3} ; s^{\varepsilon}\right),
\end{array}
$$

estimate (5.10) implies the inequality

$$
\begin{array}{r}
\left\|\left(h^{\varepsilon}\right)^{-1 / 2} \operatorname{curl}\left(s^{\varepsilon}\right)^{-1 / 2}\left(\mathbf{f}_{\varepsilon}-\left(W^{\varepsilon}\right)^{*} \Pi_{\varepsilon} \mathbf{f}_{\varepsilon}^{0}-\varepsilon \sum_{l=1}^{3} \Lambda_{l}^{\varepsilon} D_{l} \Pi_{\varepsilon} \mathbf{f}_{\varepsilon}^{0}\right)\right\|_{\mathfrak{G}} \leq \mathcal{C}_{4}^{\prime} \varepsilon\left\|s^{-1}\right\|_{L_{\infty}}^{1 / 2}\|\mathbf{F}\|_{\mathfrak{G}}, \\
0<\varepsilon \leq 1 .
\end{array}
$$

Hence,

$$
\left\|\mathbf{p}_{\varepsilon}-\widetilde{\mathbf{p}}_{\varepsilon}\right\|_{\mathfrak{G}} \leq \mathcal{C}_{4}^{\prime} \varepsilon\left\|s^{-1}\right\|_{L_{\infty}}^{1 / 2}\left\|h^{-1}\right\|_{L_{\infty}}^{1 / 2}\|\mathbf{F}\|_{\mathfrak{G}}, \quad 0<\varepsilon \leq 1,
$$


where

$$
\begin{aligned}
\widetilde{\mathbf{p}}_{\varepsilon} & =\widetilde{\mathbf{p}}_{\varepsilon}^{(1)}+\widetilde{\mathbf{p}}_{\varepsilon}^{(2)}, \\
\widetilde{\mathbf{p}}_{\varepsilon}^{(1)} & =\left(h^{\varepsilon}\right)^{-1} \operatorname{curl}\left(\left(s^{\varepsilon}\right)^{-1 / 2}\left(W^{\varepsilon}\right)^{*} \Pi_{\varepsilon} \mathbf{f}_{\varepsilon}^{0}\right), \\
\widetilde{\mathbf{p}}_{\varepsilon}^{(2)} & =\varepsilon\left(h^{\varepsilon}\right)^{-1} \operatorname{curl}\left(\left(s^{\varepsilon}\right)^{-1 / 2} \sum_{l=1}^{3} \Lambda_{l}^{\varepsilon} D_{l} \Pi_{\varepsilon} \mathbf{f}_{\varepsilon}^{0}\right) .
\end{aligned}
$$

We transform the right-hand side of (5.9) and also the expressions for the vector-valued functions (5.14) and (5.15). As was shown in [Su2, Subsection 10.2], we have

$$
s(\mathbf{x})^{-1 / 2} W(\mathbf{x})^{*}=\left(\mathbf{1}+Y_{s}(\mathbf{x})\right)\left(s^{0}\right)^{-1 / 2},
$$

where $Y_{s}(\mathbf{x})$ is the matrix with the columns $\nabla \Phi_{\mathbf{e}_{j}}(\mathbf{x}), j=1,2,3$ (this matrix was already introduced in Subsection 2.4). Therefore, the right-hand side of (5.9) can be rewritten as $\mathcal{P}\left(s^{0}\right)\left(s^{0}\right)^{-1 / 2}\left(\mathbf{1}+\left(Y_{s}^{\varepsilon}\right)^{*}\right) \mathbf{F}$. Next, let $\mathfrak{G}\left(\left(s^{0}\right)^{-1}\right)$ be the weighted $L_{2}$-space of $\mathbb{C}^{3}$ valued functions in $\mathbb{R}^{3}$ with the inner product $(\mathbf{u}, \mathbf{v})_{\mathfrak{G}\left(\left(s^{0}\right)^{-1}\right)}=\left(\left(s^{0}\right)^{-1} \mathbf{u}, \mathbf{v}\right)_{\mathfrak{G}}$. We have $\mathcal{P}\left(s^{0}\right)\left(s^{0}\right)^{-1 / 2}=\left(s^{0}\right)^{-1 / 2} \widetilde{\mathcal{P}}\left(s^{0}\right)$, where $\widetilde{\mathcal{P}}\left(s^{0}\right)$ is the orthogonal projection in the weighted space $\mathfrak{G}\left(\left(s^{0}\right)^{-1}\right)$ onto the divergence-free subspace $J$ (see [Su2, Subsection 10.2]). Then the right-hand side of $(5.9)$ takes the form $\left(s^{0}\right)^{-1 / 2} \widetilde{\mathcal{P}}\left(s^{0}\right)\left(\mathbf{1}+\left(Y_{s}^{\varepsilon}\right)^{*}\right) \mathbf{F}$. As a result, problem (5.9) can be rewritten as

$$
\begin{aligned}
\left(s^{0}\right)^{-1 / 2} \operatorname{curl}\left(h^{0}\right)^{-1} \operatorname{curl}\left(s^{0}\right)^{-1 / 2} \mathbf{f}_{\varepsilon}^{0}+\mathbf{f}_{\varepsilon}^{0} & =\left(s^{0}\right)^{-1 / 2} \widetilde{\mathcal{P}}\left(s^{0}\right)\left(\mathbf{1}+\left(Y_{s}^{\varepsilon}\right)^{*}\right) \mathbf{F}, \\
\operatorname{div}\left(s^{0}\right)^{1 / 2} \mathbf{f}_{\varepsilon}^{0} & =0 .
\end{aligned}
$$

Identity (5.16) shows that the vector-valued function (5.14) can be rewritten as

$$
\widetilde{\mathbf{p}}_{\varepsilon}^{(1)}=\left(h^{\varepsilon}\right)^{-1} \operatorname{curl}\left(\left(\mathbf{1}+Y_{s}^{\varepsilon}\right)\left(s^{0}\right)^{-1 / 2} \Pi_{\varepsilon} \mathbf{f}_{\varepsilon}^{0}\right) .
$$

Writing $\mathbf{f}_{\varepsilon}^{0}=\sum_{j=1}^{3}\left(\mathbf{f}_{\varepsilon}^{0}\right)^{(j)} \mathbf{e}_{j}$ and calculating the curl of the product, we obtain

$$
\begin{aligned}
\widetilde{\mathbf{p}}_{\varepsilon}^{(1)}= & \left(h^{\varepsilon}\right)^{-1} \sum_{j=1}^{3} \Pi_{\varepsilon}\left(\mathbf{f}_{\varepsilon}^{0}\right)^{(j)} \operatorname{curl}\left(\left(\mathbf{1}+Y_{s}^{\varepsilon}\right)\left(s^{0}\right)^{-1 / 2} \mathbf{e}_{j}\right) \\
& +\left(h^{\varepsilon}\right)^{-1} \sum_{j=1}^{3}\left(\nabla\left(\Pi_{\varepsilon}\left(\mathbf{f}_{\varepsilon}^{0}\right)^{(j)}\right)\right) \times\left(\left(\mathbf{1}+Y_{s}^{\varepsilon}\right)\left(s^{0}\right)^{-1 / 2} \mathbf{e}_{j}\right) .
\end{aligned}
$$

The first term on the right is equal to zero, because the matrix $Y_{s}$ consists of the gradients $\nabla \Phi_{\mathbf{e}_{j}}$. Hence,

$$
\widetilde{\mathbf{p}}_{\varepsilon}^{(1)}=\left(h^{\varepsilon}\right)^{-1} \sum_{j=1}^{3}\left(\nabla\left(\Pi_{\varepsilon}\left(\mathbf{f}_{\varepsilon}^{0}\right)^{(j)}\right)\right) \times\left(\left(\mathbf{1}+Y_{s}^{\varepsilon}\right)\left(s^{0}\right)^{-1 / 2} \mathbf{e}_{j}\right) .
$$

Now we transform the vector-valued function (5.15). We write the curl operator as

$$
\operatorname{curl}=\sum_{j=1}^{3} b_{j} D_{j}
$$

where

$$
b_{1}=\left(\begin{array}{ccc}
0 & 0 & 0 \\
0 & 0 & -i \\
0 & i & 0
\end{array}\right), \quad b_{2}=\left(\begin{array}{ccc}
0 & 0 & i \\
0 & 0 & 0 \\
-i & 0 & 0
\end{array}\right), \quad b_{3}=\left(\begin{array}{ccc}
0 & -i & 0 \\
i & 0 & 0 \\
0 & 0 & 0
\end{array}\right)
$$


Then expression (5.15) can be written as

$$
\begin{aligned}
& \widetilde{\mathbf{p}}_{\varepsilon}^{(2)}=\widehat{\mathbf{p}}_{\varepsilon}^{(2)}+\check{\mathbf{p}}_{\varepsilon}^{(2)}, \\
& \widehat{\mathbf{p}}_{\varepsilon}^{(2)}=\varepsilon\left(h^{\varepsilon}\right)^{-1} \sum_{l=1}^{3}\left[\operatorname{curl}\left(s^{\varepsilon}\right)^{-1 / 2} \Lambda_{l}^{\varepsilon}\right] D_{l} \Pi_{\varepsilon} \mathbf{f}_{\varepsilon}^{0}, \\
& \check{\mathbf{p}}_{\varepsilon}^{(2)}=\varepsilon \sum_{l, j=1}^{3}\left(h^{\varepsilon}\right)^{-1} b_{j}\left(s^{\varepsilon}\right)^{-1 / 2} \Lambda_{l}^{\varepsilon}\left(D_{j} D_{l} \Pi_{\varepsilon} \mathbf{f}_{\varepsilon}^{0}\right) .
\end{aligned}
$$

By $\left[\operatorname{curl}\left(s^{\varepsilon}\right)^{-1 / 2} \Lambda_{l}^{\varepsilon}\right]$ we denote the matrix with the columns

$$
\operatorname{curl}\left(\left(s^{\varepsilon}\right)^{-1 / 2} \mathbf{v}_{l j}^{\varepsilon}\right), \quad j=1,2,3 .
$$

Our goal is to show that the term (5.21) "incorporates in the remainder part"; i.e., it is dominated by $C \varepsilon\|\mathbf{F}\|_{\mathfrak{G}}$.

Lemma 5.3. For the term (5.21) we have

$$
\left\|\check{\mathbf{p}}_{\varepsilon}^{(2)}\right\|_{\mathfrak{G}} \leq C_{5} \varepsilon\|\mathbf{F}\|_{\mathfrak{G}} .
$$

The constant $C_{5}$ is defined below in (5.28).

Proof. We have

$$
\left\|\check{\mathbf{p}}_{\varepsilon}^{(2)}\right\|_{\mathfrak{G}} \leq \varepsilon\left\|h^{-1}\right\|_{L_{\infty}}\left\|s^{-1}\right\|_{L_{\infty}}^{1 / 2} \sum_{l, j=1}^{3}\left\|\Lambda_{l}^{\varepsilon}\left(D_{j} D_{l} \Pi_{\varepsilon} \mathbf{f}_{\varepsilon}^{0}\right)\right\|_{\mathfrak{G}} .
$$

From (5.17) we see that

$$
\mathbf{f}_{\varepsilon}^{0}=\left(\mathcal{L}^{0}+I\right)^{-1}\left(s^{0}\right)^{-1 / 2} \widetilde{\mathcal{P}}\left(s^{0}\right)\left(\mathbf{1}+\left(Y_{s}^{\varepsilon}\right)^{*}\right) \mathbf{F} .
$$

For the proof of inequality (5.22), it suffices to estimate the norm of the vector-valued function

$$
\begin{aligned}
\Lambda_{l}^{\varepsilon}\left(D_{j} D_{l} \Pi_{\varepsilon} \mathbf{f}_{\varepsilon}^{0}\right) & =\Lambda_{l}^{\varepsilon} D_{j} D_{l} \Pi_{\varepsilon}\left(\mathcal{L}^{0}+I\right)^{-1}\left(s^{0}\right)^{-1 / 2} \widetilde{\mathcal{P}}\left(s^{0}\right)\left(\mathbf{1}+\left(Y_{s}^{\varepsilon}\right)^{*}\right) \mathbf{F} \\
& =\left(\Lambda_{l}^{\varepsilon} \Pi_{\varepsilon}\right)\left(D_{j} D_{l}\left(\mathcal{L}^{0}+I\right)^{-1}\right)\left(s^{0}\right)^{-1 / 2} \widetilde{\mathcal{P}}\left(s^{0}\right)\left(\Pi_{\varepsilon}\left(\mathbf{1}+\left(Y_{s}^{\varepsilon}\right)^{*}\right)\right) \mathbf{F}
\end{aligned}
$$

by $C\|\mathbf{F}\|_{\mathfrak{G}}$. Recall that the symbol of the operator $\mathcal{L}^{0}$ is estimated from below by $c_{*}|\boldsymbol{\xi}|^{2}$. Consequently,

$$
\left\|D_{j} D_{l}\left(\mathcal{L}^{0}+I\right)^{-1}\right\|_{\mathfrak{G} \rightarrow \mathfrak{G}} \leq \sup _{\boldsymbol{\xi} \in \mathbb{R}^{3}}\left|\xi_{j} \xi_{l}\right|\left(c_{*}|\boldsymbol{\xi}|^{2}+1\right)^{-1} \leq c_{*}^{-1} .
$$

Next, applying a scale transformation and the Gelfand transformation, we obtain

$$
\begin{aligned}
\left\|\left[\Lambda_{l}^{\varepsilon}\right] \Pi_{\varepsilon}\right\|_{\mathfrak{G} \rightarrow \mathfrak{G}} & =\left\|\left[\Lambda_{l}\right] \Pi\right\|_{\mathfrak{G} \rightarrow \mathfrak{G}}=\left\|\left[\Lambda_{l}\right] P_{0}\right\|_{\mathfrak{H} \rightarrow \mathfrak{H}} \\
& =|\Omega|^{-1 / 2}\left\|\Lambda_{l}\right\|_{L_{2}(\Omega)}=|\Omega|^{-1 / 2}\left(\sum_{j=1}^{3}\left\|\mathbf{v}_{l j}\right\|_{\mathfrak{H}}^{2}\right)^{1 / 2}
\end{aligned}
$$

In order to estimate the norm of the solutions $\mathbf{v}_{l j}$ of problem (2.19), we use (3.24) with $\boldsymbol{\theta}=\mathbf{e}_{l}$ and $\mathbf{f}=\mathbf{g}_{j}$ and take (2.7) and (2.9) into account. We have

$$
\left\|\mathbf{v}_{l j}\right\|_{\mathfrak{H}} \leq\left(C_{2} C_{0}(s)\right)^{1 / 2}\left(2 c_{*}\right)^{-1 / 2} r_{0}^{-1}\left\|\mathbf{g}_{j}\right\|_{\mathfrak{H}} .
$$

Finally, the normalization condition $\left\|\mathbf{g}_{j}\right\|_{\mathfrak{H}}=|\Omega|^{1 / 2}$ yields

$$
\left\|\left[\Lambda_{l}^{\varepsilon}\right] \Pi_{\varepsilon}\right\|_{\mathfrak{G} \rightarrow \mathfrak{G}} \leq\left(3 C_{2} C_{0}(s)\right)^{1 / 2}\left(2 c_{*}\right)^{-1 / 2} r_{0}^{-1} .
$$


Similarly, we have

$$
\begin{aligned}
\left\|\Pi_{\varepsilon}\left[\mathbf{1}+\left(Y_{s}^{\varepsilon}\right)^{*}\right]\right\|_{\mathfrak{G} \rightarrow \mathfrak{G}} & =\left\|\left[\mathbf{1}+Y_{s}\right] \Pi\right\|_{\mathfrak{G} \rightarrow \mathfrak{G}}=\left\|\left[\mathbf{1}+Y_{s}\right] P_{0}\right\|_{\mathfrak{H} \rightarrow \mathfrak{H}} \\
& =|\Omega|^{-1 / 2}\left\|\mathbf{1}+Y_{s}\right\|_{L_{2}(\Omega)}=|\Omega|^{-1 / 2}\left(\sum_{j=1}^{3}\left\|\mathbf{e}_{j}+\nabla \Phi_{\mathbf{e}_{j}}\right\|_{\mathfrak{H}}^{2}\right)^{1 / 2} .
\end{aligned}
$$

The equation $\operatorname{div} s(\mathbf{x})\left(\mathbf{e}_{j}+\nabla \Phi_{\mathbf{e}_{j}}\right)=0$ implies

$$
\left\|s^{1 / 2}\left(\mathbf{e}_{j}+\nabla \Phi_{\mathbf{e}_{j}}\right)\right\|_{\mathfrak{H}} \leq\left\|s^{1 / 2} \mathbf{e}_{j}\right\|_{\mathfrak{H}},
$$

whence

$$
\left\|\mathbf{e}_{j}+\nabla \Phi_{\mathbf{e}_{j}}\right\|_{\mathfrak{H}} \leq\|s\|_{L_{\infty}}^{1 / 2}\left\|s^{-1}\right\|_{L_{\infty}}^{1 / 2}|\Omega|^{1 / 2}
$$

As a result, we obtain

$$
\left\|\Pi_{\varepsilon}\left[\mathbf{1}+\left(Y_{s}^{\varepsilon}\right)^{*}\right]\right\|_{\mathfrak{G} \rightarrow \mathfrak{G}} \leq \sqrt{3}\|s\|_{L_{\infty}^{1 / 2}}^{1 / 2} s^{-1} \|_{L_{\infty}}^{1 / 2} .
$$

Now (5.24)-(5.27) imply

$$
\left\|\Lambda_{l}^{\varepsilon}\left(D_{j} D_{l} \Pi_{\varepsilon} \mathbf{f}_{\varepsilon}^{0}\right)\right\|_{\mathfrak{G}} \leq(3 / \sqrt{2})\left(C_{2} C_{0}(s)\right)^{1 / 2} c_{*}^{-3 / 2} r_{0}^{-1}\|s\|_{L_{\infty}}^{1 / 2}\left\|s^{-1}\right\|_{L_{\infty}}\|\mathbf{F}\|_{\mathfrak{G}} .
$$

Combining this with (5.23), we arrive at the required estimate (5.22) with the constant

$$
C_{5}=(27 / \sqrt{2})\left(C_{2} C_{0}(s)\right)^{1 / 2} c_{*}^{-3 / 2} r_{0}^{-1}\left\|h^{-1}\right\|_{L_{\infty}}\|s\|_{L_{\infty}}^{1 / 2}\left\|s^{-1}\right\|_{L_{\infty}}^{3 / 2} .
$$

It remains to analyze the term (5.20), which can be rewritten as

$$
\widehat{\mathbf{p}}_{\varepsilon}^{(2)}=\left(h^{\varepsilon}\right)^{-1} \sum_{l=1}^{3}\left[\left(\operatorname{curl} s^{-1 / 2} \Lambda_{l}\right)^{\varepsilon}\right] D_{l} \Pi_{\varepsilon} \mathbf{f}_{\varepsilon}^{0} .
$$

Here curl $s^{-1 / 2} \Lambda_{l}$ is the matrix with the columns $\operatorname{curl}\left(s(\mathbf{x})^{-1 / 2} \mathbf{v}_{l j}(\mathbf{x})\right), j=1,2,3$. By (2.22), (2.24), and (2.25), we have

$$
\begin{aligned}
\operatorname{curl} s^{-1 / 2} \mathbf{v}_{l j} & =i h\left(\widehat{\mathbf{C}}_{l j}+\nabla \Psi_{\widehat{\mathbf{C}}_{l j}}\right)-i \mathbf{e}_{l} \times\left(s^{-1 / 2} \mathbf{g}_{j}\right) \\
& =i h\left(\mathbf{1}+Y_{h}\right)\left(h^{0}\right)^{-1}\left(\mathbf{e}_{l} \times\left(\left(s^{0}\right)^{-1 / 2} \mathbf{e}_{j}\right)\right)+i\left(\left(\mathbf{1}+Y_{s}\right)\left(s^{0}\right)^{-1 / 2} \mathbf{e}_{j}\right) \times \mathbf{e}_{l} .
\end{aligned}
$$

Then $\left(h^{\varepsilon}\right)^{-1}\left(\operatorname{curl} s^{-1 / 2} \Lambda_{l}\right)^{\varepsilon}$ is the matrix with the columns

$$
\begin{array}{r}
i\left(\mathbf{1}+Y_{h}^{\varepsilon}\right)\left(h^{0}\right)^{-1}\left(\mathbf{e}_{l} \times\left(\left(s^{0}\right)^{-1 / 2} \mathbf{e}_{j}\right)\right)+i\left(h^{\varepsilon}\right)^{-1}\left(\left(\left(\mathbf{1}+Y_{s}^{\varepsilon}\right)\left(s^{0}\right)^{-1 / 2} \mathbf{e}_{j}\right) \times \mathbf{e}_{l}\right), \\
j=1,2,3 .
\end{array}
$$

Since $i \sum_{l=1}^{3} \mathbf{e}_{l} D_{l}=i \mathbf{D}=\nabla$, we have

$$
\begin{aligned}
\widehat{\mathbf{p}}_{\varepsilon}^{(2)}= & \left(\mathbf{1}+Y_{h}^{\varepsilon}\right)\left(h^{0}\right)^{-1} \operatorname{curl}\left(\left(s^{0}\right)^{-1 / 2} \Pi_{\varepsilon} \mathbf{f}_{\varepsilon}^{0}\right) \\
& +\left(h^{\varepsilon}\right)^{-1} \sum_{j=1}^{3}\left(\left(\mathbf{1}+Y_{s}^{\varepsilon}\right)\left(s^{0}\right)^{-1 / 2} \mathbf{e}_{j}\right) \times\left(\nabla\left(\Pi_{\varepsilon}\left(\mathbf{f}_{\varepsilon}^{0}\right)^{(j)}\right)\right) .
\end{aligned}
$$

Adding (5.18) and (5.29), we see that the second term in (5.29) is precisely compensated for by the expression (5.18). Thus,

$$
\widetilde{\mathbf{p}}_{\varepsilon}^{(1)}+\widehat{\mathbf{p}}_{\varepsilon}^{(2)}=\left(\mathbf{1}+Y_{h}^{\varepsilon}\right)\left(h^{0}\right)^{-1} \operatorname{curl}\left(\left(s^{0}\right)^{-1 / 2} \Pi_{\varepsilon} \mathbf{f}_{\varepsilon}^{0}\right) .
$$

Relations (5.12), (5.13), (5.19), (5.22), and (5.30) imply the following result. 
Theorem 5.4. Let $\mathbf{f}_{\varepsilon}$ be the solution of problem (5.8), and let $\mathbf{p}_{\varepsilon}$ be defined by (5.11). Put $\mathbf{a}_{\varepsilon}=\operatorname{curl}\left(s^{\varepsilon}\right)^{-1 / 2} \mathbf{f}_{\varepsilon}$. Denote by $\mathbf{f}_{\varepsilon}^{0}$ the solution of problem (5.17), and by $\Pi_{\varepsilon}$ the $\Psi D O$ (5.4). Let $Y_{h}(\mathbf{x})$ be the matrix with the columns $\nabla \Psi_{\mathbf{e}_{j}}(\mathbf{x}), j=1,2,3$, where $\Psi_{\mathbf{e}_{j}}$ is the periodic solution of equation (2.23) with $\mathbf{C}=\mathbf{e}_{j}$. Denote $\widetilde{h}(\mathbf{x})=h(\mathbf{x})\left(\mathbf{1}+Y_{h}(\mathbf{x})\right)$. Then for $0<\varepsilon \leq 1$ we have

$$
\begin{aligned}
& \| \mathbf{p}_{\varepsilon}-\left(\mathbf{1}+Y_{h}^{\varepsilon}\right)\left(h^{0}\right)^{-1} \operatorname{curl}\left(\left(s^{0}\right)^{-1 / 2} \Pi_{\varepsilon} \mathbf{f}_{\varepsilon}^{0}\right)\left\|_{\mathfrak{G}} \leq \mathcal{C}_{7} \varepsilon\right\| \mathbf{F} \|_{\mathfrak{G}}, \\
&\left\|\mathbf{a}_{\varepsilon}-\widetilde{h}^{\varepsilon}\left(h^{0}\right)^{-1} \operatorname{curl}\left(\left(s^{0}\right)^{-1 / 2} \Pi_{\varepsilon} \mathbf{f}_{\varepsilon}^{0}\right)\right\|_{\mathfrak{G}} \leq \mathcal{C}_{7}\|h\|_{L_{\infty}} \varepsilon\|\mathbf{F}\|_{\mathfrak{G}} .
\end{aligned}
$$

Here

$$
\mathcal{C}_{7}=\mathcal{C}_{4}^{\prime}\left\|s^{-1}\right\|_{L_{\infty}}^{1 / 2}\left\|h^{-1}\right\|_{L_{\infty}}^{1 / 2}+C_{5} .
$$

Inequality (5.32) follows immediately from (5.31), by multiplying the vector-valued functions under the norm sign in (5.31) by $h^{\varepsilon}$ from the left.

Remark 5.5. Since the functions $\mathbf{f}_{\varepsilon}$ and $\mathbf{f}_{\varepsilon}^{0}$ are defined in terms of the "divergence-free" parts of the operators $\mathcal{L}_{\varepsilon}$ and $\mathcal{L}^{0}$ only, and all terms under the norm sign in (5.31) are expressed, in the long run, in terms of the coefficients $s$ and $h$, we may assume that $\nu(\mathbf{x})=1$. Then the constant $\mathcal{C}_{7}$ in (5.31) (and in (5.32)) corresponds to the operator $\mathcal{L}(h, s, 1)$ and depends only on $\|h\|_{L_{\infty}},\left\|h^{-1}\right\|_{L_{\infty}},\|s\|_{L_{\infty}},\left\|s^{-1}\right\|_{L_{\infty}}$, and the parameters of the lattice $\Gamma$.

5.4. Formula (5.17) shows that the function $\mathbf{f}_{\varepsilon}^{0}$ is represented as a sum of two terms,

$$
\mathbf{f}_{\varepsilon}^{0}=\mathbf{f}_{0}+\widehat{\mathbf{f}}_{\varepsilon},
$$

where $\mathbf{f}_{0}$ does not depend on $\varepsilon$ and is the solution of the "homogenized" problem

$$
\left(s^{0}\right)^{-1 / 2} \operatorname{curl}\left(h^{0}\right)^{-1} \operatorname{curl}\left(s^{0}\right)^{-1 / 2} \mathbf{f}_{0}+\mathbf{f}_{0}=\left(s^{0}\right)^{-1 / 2} \mathbf{F}, \quad \operatorname{div}\left(s^{0}\right)^{1 / 2} \mathbf{f}_{0}=0, \quad \mathbf{F} \in J,
$$

and $\widehat{\mathbf{f}}_{\varepsilon}$ is the solution of the "correction" problem

$$
\begin{gathered}
\left(s^{0}\right)^{-1 / 2} \operatorname{curl}\left(h^{0}\right)^{-1} \operatorname{curl}\left(s^{0}\right)^{-1 / 2} \widehat{\mathbf{f}}_{\varepsilon}+\widehat{\mathbf{f}}_{\varepsilon}=\left(s^{0}\right)^{-1 / 2} \widetilde{\mathcal{P}}\left(s^{0}\right)\left(Y_{s}^{\varepsilon}\right)^{*} \mathbf{F}, \\
\operatorname{div}\left(s^{0}\right)^{1 / 2} \widehat{\mathbf{f}}_{\varepsilon}=0 .
\end{gathered}
$$

The right-hand side of the "correction" problem involves the rapidly oscillating factor $\left(Y_{s}^{\varepsilon}\right)^{*}$ with zero mean value. That is why the solution $\widehat{\mathbf{f}}_{\varepsilon}$ weakly tends to zero in $\mathfrak{G}$ as $\varepsilon \rightarrow 0$.

We introduce the notation

$$
\mathbf{p}_{0}=\left(h^{0}\right)^{-1} \operatorname{curl}\left(s^{0}\right)^{-1 / 2} \mathbf{f}_{0}
$$

for the "homogenized" flux and the notation

$$
\widehat{\mathbf{p}}_{\varepsilon}=\left(h^{0}\right)^{-1} \operatorname{curl}\left(s^{0}\right)^{-1 / 2} \widehat{\mathbf{f}}_{\varepsilon}
$$

for the "correction" flux. Then formula (5.31) can be rewritten as

$$
\left\|\mathbf{p}_{\varepsilon}-\left(\mathbf{1}+Y_{h}^{\varepsilon}\right)\left(\Pi_{\varepsilon} \mathbf{p}_{0}+\Pi_{\varepsilon} \widehat{\mathbf{p}}_{\varepsilon}\right)\right\|_{\mathfrak{G}} \leq \mathcal{C}_{7} \varepsilon\|\mathbf{F}\|_{\mathfrak{G}}, \quad 0<\varepsilon \leq 1 .
$$

Similarly, we denote

$$
\begin{aligned}
& \mathbf{a}_{0}=\operatorname{curl}\left(s^{0}\right)^{-1 / 2} \mathbf{f}_{0}, \\
& \widehat{\mathbf{a}}_{\varepsilon}=\operatorname{curl}\left(s^{0}\right)^{-1 / 2} \widehat{\mathbf{f}}_{\varepsilon} .
\end{aligned}
$$

We put

$$
G_{h}(\mathbf{x})=\widetilde{h}(\mathbf{x})\left(h^{0}\right)^{-1}-\mathbf{1}=h(\mathbf{x})\left(\mathbf{1}+Y_{h}(\mathbf{x})\right)\left(h^{0}\right)^{-1}-\mathbf{1} .
$$


Then $G_{h}(\mathbf{x})$ is the periodic matrix-valued function with zero mean value (this follows from the definition of the effective matrix $h^{0}$ ). Now formula (5.32) takes the form

$$
\left\|\mathbf{a}_{\varepsilon}-\left(\mathbf{1}+G_{h}^{\varepsilon}\right)\left(\Pi_{\varepsilon} \mathbf{a}_{0}+\Pi_{\varepsilon} \widehat{\mathbf{a}}_{\varepsilon}\right)\right\|_{\mathfrak{G}} \leq \mathcal{C}_{7}\|h\|_{L_{\infty}} \varepsilon\|\mathbf{F}\|_{\mathfrak{G}}, \quad 0<\varepsilon \leq 1 .
$$

\section{§6. Elimination OF THE SMOOTHING OPERATOR}

6.1. Elimination of $\Pi_{\varepsilon}$ from the terms involving homogenized fields. Now, we analyze the possibility of getting rid of the smoothing operator $\Pi_{\varepsilon}$ in the approximations (5.37) and (5.41). It is possible to replace $\Pi_{\varepsilon}$ by $I$ in the terms involving the "homogenized" fields $\mathbf{p}_{0}$ and $\mathbf{a}_{0}$. By (5.35) and (5.33), we have

$$
\left(\mathbf{1}+Y_{h}^{\varepsilon}\right)\left(I-\Pi_{\varepsilon}\right) \mathbf{p}_{0}=\left(\mathbf{1}+Y_{h}^{\varepsilon}\right)\left(h^{0}\right)^{-1}\left(I-\Pi_{\varepsilon}\right) \operatorname{curl}\left(s^{0}\right)^{-1 / 2}\left(\mathcal{L}^{0}+I\right)^{-1} \mathcal{P}\left(s^{0}\right)\left(s^{0}\right)^{-1 / 2} \mathbf{F} .
$$

Applying a scale transformation, we obtain

$$
\left(\mathbf{1}+Y_{h}^{\varepsilon}\right)\left(I-\Pi_{\varepsilon}\right) \mathbf{p}_{0}=\varepsilon\left(T_{\varepsilon}^{*}\left(\mathbf{1}+Y_{h}\right)\left(h^{0}\right)^{-1} \Sigma(\varepsilon) T_{\varepsilon}\right) \mathcal{P}\left(s^{0}\right)\left(s^{0}\right)^{-1 / 2} \mathbf{F},
$$

where

$$
\Sigma(\varepsilon)=(I-\Pi) \operatorname{curl}\left(s^{0}\right)^{-1 / 2}\left(\mathcal{L}^{0}+\varepsilon^{2} I\right)^{-1} .
$$

Similarly, (5.38) and (5.33) imply

$$
\left(\mathbf{1}+G_{h}^{\varepsilon}\right)\left(I-\Pi_{\varepsilon}\right) \mathbf{a}_{0}=\varepsilon\left(T_{\varepsilon}^{*}\left(\mathbf{1}+G_{h}\right) \Sigma(\varepsilon) T_{\varepsilon}\right) \mathcal{P}\left(s^{0}\right)\left(s^{0}\right)^{-1 / 2} \mathbf{F} .
$$

The operator $\Sigma(\varepsilon)$ is a $\Psi$ DO of order $(-1)$; this operator maps $\mathfrak{G}$ to $\mathfrak{G}^{1}=H^{1}\left(\mathbb{R}^{3} ; \mathbb{C}^{3}\right)$ continuously. Herewith,

$$
\|\Sigma(\varepsilon)\|_{\mathfrak{G} \rightarrow \mathfrak{G}^{1}}=\sup _{\boldsymbol{\xi} \in \mathbb{R}^{3}}\left|1-\chi_{\widetilde{\Omega}}(\boldsymbol{\xi}) \| r(\boldsymbol{\xi})\left(s^{0}\right)^{-1 / 2}\left(S^{0}(\boldsymbol{\xi})+\varepsilon^{2} \mathbf{1}\right)^{-1}\right|\left(1+|\boldsymbol{\xi}|^{2}\right)^{1 / 2} .
$$

Recall that $r(\boldsymbol{\xi})$ is the symbol of the operator $-i$ curl, and $|r(\boldsymbol{\xi})| \leq|\boldsymbol{\xi}|$. The symbol of the operator $\mathcal{L}^{0}$ is $S^{0}(\boldsymbol{\xi})$, and $S^{0}(\boldsymbol{\xi}) \geq c_{*}|\boldsymbol{\xi}|^{2} \mathbf{1}$. We have

$$
\begin{aligned}
\|\Sigma(\varepsilon)\|_{\mathfrak{G} \rightarrow \mathfrak{G}^{1}} & \leq\left|\left(s^{0}\right)^{-1 / 2}\right| \sup _{|\boldsymbol{\xi}| \geq r_{0}}|\boldsymbol{\xi}|\left(c_{*}|\boldsymbol{\xi}|^{2}+\varepsilon^{2}\right)^{-1}\left(1+|\boldsymbol{\xi}|^{2}\right)^{1 / 2} \\
& \leq\left\|s^{-1}\right\|_{L_{\infty}}^{1 / 2} c_{*}^{-1}\left(1+r_{0}^{-2}\right)^{1 / 2} .
\end{aligned}
$$

As was shown in [Su2, Proposition 8.2], the columns of the matrix $Y_{h}(\mathbf{x})$ (the gradients of the periodic solutions of (2.23)) are multipliers from $H^{1}\left(\mathbb{R}^{3}\right)$ to $L_{2}\left(\mathbb{R}^{3}\right)$. Then

$$
\left\|\left[Y_{h}\right]\right\|_{\mathfrak{G}^{1} \rightarrow \mathfrak{G}} \leq \mathfrak{C}_{1}(h) .
$$

By (5.40), we also have

$$
\left\|\left[G_{h}\right]\right\|_{\mathfrak{G}^{1} \rightarrow \mathfrak{G}} \leq \mathfrak{C}_{2}(h) .
$$

The constants $\mathfrak{C}_{1}(h)$ and $\mathfrak{C}_{2}(h)$ depend only on $\|h\|_{L_{\infty}},\left\|h^{-1}\right\|_{L_{\infty}}$, and on the parameters of the lattice $\Gamma$.

Now relations (6.1), (6.3), and (6.4) imply

$$
\begin{aligned}
\|(\mathbf{1} & \left.+Y_{h}^{\varepsilon}\right)\left(I-\Pi_{\varepsilon}\right) \mathbf{p}_{0} \|_{\mathfrak{G}} \\
& \leq \varepsilon\left|\left(h^{0}\right)^{-1}\left\|\left(s^{0}\right)^{-1 / 2} \mid\left(1+\left\|\left[Y_{h}\right]\right\|_{\mathfrak{G}^{1} \rightarrow \mathfrak{G}}\right)\right\| \Sigma(\varepsilon)\left\|_{\mathfrak{G} \rightarrow \mathfrak{G}^{1}}\right\| \mathbf{F} \|_{\mathfrak{G}}\right. \\
& \leq \varepsilon\left\|h^{-1}\right\|_{L_{\infty}}\left\|s^{-1}\right\|_{L_{\infty}}\left(1+\mathfrak{C}_{1}(h)\right) c_{*}^{-1}\left(1+r_{0}^{-2}\right)^{1 / 2}\|\mathbf{F}\|_{\mathfrak{G}} .
\end{aligned}
$$

Similarly, (6.2), (6.3), and (6.5) yield

$$
\left\|\left(\mathbf{1}+G_{h}^{\varepsilon}\right)\left(I-\Pi_{\varepsilon}\right) \mathbf{a}_{0}\right\|_{\mathfrak{G}} \leq \varepsilon\left\|s^{-1}\right\|_{L_{\infty}}\left(1+\mathfrak{C}_{2}(h)\right) c_{*}^{-1}\left(1+r_{0}^{-2}\right)^{1 / 2}\|\mathbf{F}\|_{\mathfrak{G} .} .
$$

As a result, from (5.37) and (6.6) we deduce that

$$
\left\|\mathbf{p}_{\varepsilon}-\left(\mathbf{1}+Y_{h}^{\varepsilon}\right)\left(\mathbf{p}_{0}+\Pi_{\varepsilon} \widehat{\mathbf{p}}_{\varepsilon}\right)\right\|_{\mathfrak{G}} \leq \mathcal{C}_{8} \varepsilon\|\mathbf{F}\|_{\mathfrak{G}}, \quad 0<\varepsilon \leq 1,
$$


where

$$
\mathcal{C}_{8}=\mathcal{C}_{7}+\left\|h^{-1}\right\|_{L_{\infty}}\left\|s^{-1}\right\|_{L_{\infty}}\left(1+\mathfrak{C}_{1}(h)\right) c_{*}^{-1}\left(1+r_{0}^{-2}\right)^{1 / 2} .
$$

Inequalities (5.41) and (6.7) imply

$$
\left\|\mathbf{a}_{\varepsilon}-\left(\mathbf{1}+G_{h}^{\varepsilon}\right)\left(\mathbf{a}_{0}+\Pi_{\varepsilon} \widehat{\mathbf{a}}_{\varepsilon}\right)\right\|_{\mathfrak{G}} \leq \mathcal{C}_{9} \varepsilon\|\mathbf{F}\|_{\mathfrak{G}}, \quad 0<\varepsilon \leq 1,
$$

where

$$
\mathcal{C}_{9}=\mathcal{C}_{7}\|h\|_{L_{\infty}}+\left\|s^{-1}\right\|_{L_{\infty}}\left(1+\mathfrak{C}_{2}(h)\right) c_{*}^{-1}\left(1+r_{0}^{-2}\right)^{1 / 2} .
$$

Thus, an approximation for the flux $\mathbf{p}_{\varepsilon}$ is given by the sum of four terms

$$
\left(\mathbf{1}+Y_{h}^{\varepsilon}\right)\left(\mathbf{p}_{0}+\Pi_{\varepsilon} \widehat{\mathbf{p}}_{\varepsilon}\right)=\mathbf{p}_{0}+Y_{h}^{\varepsilon} \mathbf{p}_{0}+\Pi_{\varepsilon} \widehat{\mathbf{p}}_{\varepsilon}+Y_{h}^{\varepsilon} \Pi_{\varepsilon} \widehat{\mathbf{p}}_{\varepsilon},
$$

where the leading term $\mathbf{p}_{0}$ does not depend on $\varepsilon$ and is the "homogenized" flux (see (5.35)). The remaining three terms involve rapidly oscillating factors with zero mean. It can be shown that these three terms converge weakly to zero in $\mathfrak{G}$ as $\varepsilon \rightarrow 0$. These terms play the role of correctors of zero order.

6.2. Elimination of $\Pi_{\varepsilon}$ from the terms $\Pi_{\varepsilon} \widetilde{\mathbf{p}}_{\varepsilon}$ and $\Pi_{\varepsilon} \widetilde{\mathbf{a}}_{\varepsilon}$. It is possible to replace $\Pi_{\varepsilon}$ by $I$ in the third term on the right-hand side of (6.10). Indeed, by (5.36) and (5.34), we have

$$
\left(I-\Pi_{\varepsilon}\right) \widehat{\mathbf{p}}_{\varepsilon}=\left(I-\Pi_{\varepsilon}\right)\left(h^{0}\right)^{-1} \operatorname{curl}\left(s^{0}\right)^{-1 / 2}\left(\mathcal{L}^{0}+I\right)^{-1} \mathcal{P}\left(s^{0}\right)\left(s^{0}\right)^{-1 / 2}\left(Y_{s}^{\varepsilon}\right)^{*} \mathbf{F} .
$$

Then

$$
\left(I-\Pi_{\varepsilon}\right) \widehat{\mathbf{p}}_{\varepsilon}=\varepsilon\left(T_{\varepsilon}^{*}\left(h^{0}\right)^{-1} \Sigma(\varepsilon) \mathcal{P}\left(s^{0}\right)\left(s^{0}\right)^{-1 / 2} Y_{s}^{*} T_{\varepsilon}\right) \mathbf{F} .
$$

Passing to adjoint operators, we arrive at the estimate

$$
\left\|\left(I-\Pi_{\varepsilon}\right) \widehat{\mathbf{p}}_{\varepsilon}\right\|_{\mathfrak{G}} \leq \varepsilon\left|\left(h^{0}\right)^{-1}\left\|\left(s^{0}\right)^{-1 / 2} \mid\right\| \Sigma(\varepsilon)^{*}\left\|_{\mathfrak{G} \rightarrow \mathfrak{G}^{1}}\right\|\left[Y_{s}\right]\left\|_{\mathfrak{G}^{1} \rightarrow \mathfrak{G}}\right\| \mathbf{F} \|_{\mathfrak{G} .}\right.
$$

The operator $\Sigma(\varepsilon)^{*}=\left(\mathcal{L}^{0}+\varepsilon^{2} I\right)^{-1}\left(s^{0}\right)^{-1 / 2} \operatorname{curl}(I-\Pi)$ admits the same estimate in the $\left(\mathfrak{G} \rightarrow \mathfrak{G}^{1}\right.$ )-operator norm as the operator $\Sigma(\varepsilon)$ (see (6.3)). The matrix-valued function $Y_{s}$ is a multiplier from $\mathfrak{G}^{1}$ to $\mathfrak{G}$, and $\left\|\left[Y_{s}\right]\right\|_{\mathfrak{G}^{1} \rightarrow \mathfrak{G}} \leq \mathfrak{C}_{1}(s)$ (cf. (6.4)). As a result, we obtain

$$
\left\|\left(I-\Pi_{\varepsilon}\right) \widehat{\mathbf{p}}_{\varepsilon}\right\|_{\mathfrak{G}} \leq \varepsilon\left\|h^{-1}\right\|_{L_{\infty}}\left\|s^{-1}\right\|_{L_{\infty}} c_{*}^{-1}\left(1+r_{0}^{-2}\right)^{1 / 2} \mathfrak{C}_{1}(s)\|\mathbf{F}\|_{\mathfrak{G}} .
$$

Similarly, we have

$$
\left\|\left(I-\Pi_{\varepsilon}\right) \widehat{\mathbf{a}}_{\varepsilon}\right\|_{\mathfrak{G}} \leq \varepsilon\left\|s^{-1}\right\|_{L_{\infty}} c_{*}^{-1}\left(1+r_{0}^{-2}\right)^{1 / 2} \mathfrak{C}_{1}(s)\|\mathbf{F}\|_{\mathfrak{G}} .
$$

Inequalities (6.8) and (6.12) imply

$$
\left\|\mathbf{p}_{\varepsilon}-\left(\mathbf{p}_{0}+Y_{h}^{\varepsilon} \mathbf{p}_{0}+\widehat{\mathbf{p}}_{\varepsilon}+Y_{h}^{\varepsilon} \Pi_{\varepsilon} \widehat{\mathbf{p}}_{\varepsilon}\right)\right\|_{\mathfrak{G}} \leq \check{\mathcal{C}}_{8} \varepsilon\|\mathbf{F}\|_{\mathfrak{G}}, \quad 0<\varepsilon \leq 1,
$$

where

$$
\check{\mathcal{C}}_{8}=\mathcal{C}_{8}+\left\|h^{-1}\right\|_{L_{\infty}}\left\|s^{-1}\right\|_{L_{\infty}} c_{*}^{-1}\left(1+r_{0}^{-2}\right)^{1 / 2} \mathfrak{C}_{1}(s) .
$$

Similarly, by (6.9) and (6.13),

$$
\left\|\mathbf{a}_{\varepsilon}-\left(\mathbf{a}_{0}+G_{h}^{\varepsilon} \mathbf{a}_{0}+\widehat{\mathbf{a}}_{\varepsilon}+G_{h}^{\varepsilon} \Pi_{\varepsilon} \widehat{\mathbf{a}}_{\varepsilon}\right)\right\|_{\mathfrak{G}} \leq \check{\mathcal{C}}_{9} \varepsilon\|\mathbf{F}\|_{\mathfrak{G}}, \quad 0<\varepsilon \leq 1,
$$

where

$$
\check{\mathcal{C}}_{9}=\mathcal{C}_{9}+\left\|s^{-1}\right\|_{L_{\infty}} c_{*}^{-1}\left(1+r_{0}^{-2}\right)^{1 / 2} \mathfrak{C}_{1}(s) .
$$

We have proved the following result. 
Theorem 6.1. Let $\mathbf{f}_{\varepsilon}$ be the solution of problem (5.8), and let $\mathbf{p}_{\varepsilon}$ be defined by (5.11). Denote $\mathbf{a}_{\varepsilon}=\operatorname{curl}\left(s^{\varepsilon}\right)^{-1 / 2} \mathbf{f}_{\varepsilon}$. Consider the solution $\mathbf{f}_{0}$ of problem (5.33). Let $\mathbf{p}_{0}$ be defined by (5.35), and let $\mathbf{a}_{0}$ be defined by (5.38). Denote by $\widehat{\mathbf{f}}_{\varepsilon}$ the solution of problem (5.34). Let $\widehat{\mathbf{p}}_{\varepsilon}$ be defined by (5.36), and let $\widehat{\mathbf{a}}_{\varepsilon}$ be defined by (5.39). Let $\Pi_{\varepsilon}$ be the $\Psi D O$ (5.4). Consider the matrix $Y_{h}(\mathbf{x})$ with the columns $\nabla \Psi_{\mathbf{e}_{j}}(\mathbf{x}), j=1,2,3$, where $\Psi_{\mathbf{e}_{j}}$ is the periodic solution of equation (2.23) with $\mathbf{C}=\mathbf{e}_{j}$. Let $G_{h}(\mathbf{x})$ be the matrix defined by (5.40). Then estimates (6.14) and (6.15) are true for $0<\varepsilon \leq 1$. The constants $\check{\mathcal{C}}_{8}$ and $\check{\mathcal{C}}_{9}$ depend only on the norms $\|s\|_{L_{\infty}},\left\|s^{-1}\right\|_{L_{\infty}},\|h\|_{L_{\infty}},\left\|h^{-1}\right\|_{L_{\infty}}$, and on the parameters of the lattice $\Gamma$.

6.3. On the possibility of elimination of $\Pi_{\varepsilon}$ from the terms $Y_{h}^{\varepsilon} \Pi_{\varepsilon} \widetilde{\mathbf{p}}_{\varepsilon}$ and $G_{h}^{\varepsilon} \Pi_{\varepsilon} \widetilde{\mathbf{a}}_{\varepsilon}$. Note that the right-hand side of (5.34) is equal to $\mathcal{P}\left(s^{0}\right)\left(s^{0}\right)^{-1 / 2}\left(Y_{s}^{\varepsilon}\right)^{*} \mathbf{F}$ and belongs to $H^{-1}\left(\mathbb{R}^{3} ; \mathbb{C}^{3}\right)$. Indeed, $\mathbf{F} \in L_{2}\left(\mathbb{R}^{3} ; \mathbb{C}^{3}\right)$, and $\left(Y_{s}^{\varepsilon}\right)^{*}$ is a multiplier from $L_{2}$ to $H^{-1}$. Moreover, the operator $\mathcal{P}\left(s^{0}\right)$ extends up to a continuous operator (projection) in $H^{-1}\left(\mathbb{R}^{3} ; \mathbb{C}^{3}\right)$ (see [BSu2, Chapter 7, Subsection 2.4]). Then we have $\widehat{\mathbf{f}}_{\varepsilon} \in H^{1}\left(\mathbb{R}^{3} ; \mathbb{C}^{3}\right)$ and, by (5.36), $\widehat{\mathbf{p}}_{\varepsilon} \in L_{2}\left(\mathbb{R}^{3} ; \mathbb{C}^{3}\right)$. Since $Y_{h}^{\varepsilon}$ is a multiplier from $L_{2}$ to $H^{-1}$, the term $Y_{h}^{\varepsilon} \widehat{\mathbf{p}}_{\varepsilon}$ belongs to $H^{-1}\left(\mathbb{R}^{3} ; \mathbb{C}^{3}\right)$. In the general case, we cannot guarantee that the term $Y_{h}^{\varepsilon} \widehat{\mathbf{p}}_{\varepsilon}$ belongs to $L_{2}$. Therefore, in general, for $L_{2}$-approximations we cannot avoid the smoothing operator in the last terms in (6.14) and (6.15).

However, under some additional restrictions on the coefficients, it is possible to eliminate the operator $\Pi_{\varepsilon}$ from the last term in (6.14) and in (6.15). Suppose that the matrix $s(\mathbf{x})$ is such that

$$
\nabla \Phi_{\mathbf{e}_{j}} \in L_{\infty}, \quad j=1,2,3,
$$

where $\Phi_{\mathbf{e}_{j}}$ is the $\Gamma$-periodic solution of equation (2.1) with $\mathbf{C}=\mathbf{e}_{j}$. Then $Y_{s} \in L_{\infty}$. We use (6.3) to estimate the $\mathfrak{G}^{1}$-norm of the vector-valued function (6.11):

$$
\begin{aligned}
\left\|\left(I-\Pi_{\varepsilon}\right) \widehat{\mathbf{p}}_{\varepsilon}\right\|_{\mathfrak{G}^{1}} & \leq \varepsilon\left|\left(h^{0}\right)^{-1}\left\|\left(s^{0}\right)^{-1 / 2} \mid\right\| \Sigma(\varepsilon)\left\|_{\mathfrak{G}_{\mathfrak{G} \rightarrow \mathfrak{G}^{1}}}\right\| Y_{s}\left\|_{L_{\infty}}\right\| \mathbf{F} \|_{\mathfrak{G}}\right. \\
& \leq \varepsilon\left\|h^{-1}\right\|_{L_{\infty}}\left\|s^{-1}\right\|_{L_{\infty}} c_{*}^{-1}\left(1+r_{0}^{-2}\right)^{1 / 2}\left\|Y_{s}\right\|_{L_{\infty}}\|\mathbf{F}\|_{\mathfrak{G}} .
\end{aligned}
$$

Then, by (6.4),

$$
\left\|Y_{h}^{\varepsilon}\left(I-\Pi_{\varepsilon}\right) \widehat{\mathbf{p}}_{\varepsilon}\right\|_{\mathfrak{G}} \leq \varepsilon \mathfrak{C}_{1}(h)\left\|h^{-1}\right\|_{L_{\infty}}\left\|s^{-1}\right\|_{L_{\infty}} c_{*}^{-1}\left(1+r_{0}^{-2}\right)^{1 / 2}\left\|Y_{s}\right\|_{L_{\infty}}\|\mathbf{F}\|_{\mathfrak{G}} .
$$

Similarly,

$$
\left\|G_{h}^{\varepsilon}\left(I-\Pi_{\varepsilon}\right) \widehat{\mathbf{a}}_{\varepsilon}\right\|_{\mathfrak{G}} \leq \varepsilon \mathfrak{C}_{2}(h)\left\|s^{-1}\right\|_{L_{\infty}} c_{*}^{-1}\left(1+r_{0}^{-2}\right)^{1 / 2}\left\|Y_{s}\right\|_{L_{\infty}}\|\mathbf{F}\|_{\mathfrak{G}} .
$$

We put

$$
\begin{aligned}
\widetilde{\mathcal{C}}_{8} & =\check{\mathcal{C}}_{8}+\mathfrak{C}_{1}(h)\left\|h^{-1}\right\|_{L_{\infty}}\left\|s^{-1}\right\|_{L_{\infty}} c_{*}^{-1}\left(1+r_{0}^{-2}\right)^{1 / 2}\left\|Y_{s}\right\|_{L_{\infty}}, \\
\widetilde{\mathcal{C}}_{9} & =\check{\mathcal{C}}_{9}+\mathfrak{C}_{2}(h)\left\|s^{-1}\right\|_{L_{\infty}} c_{*}^{-1}\left(1+r_{0}^{-2}\right)^{1 / 2}\left\|Y_{s}\right\|_{L_{\infty}} .
\end{aligned}
$$

Now relations (6.14), (6.15), (6.17), and (6.18) imply the following statement.

Theorem 6.2. Under the assumptions of Theorem 6.1, suppose that condition (6.16) is satisfied. Then we have

$$
\begin{aligned}
\left\|\mathbf{p}_{\varepsilon}-\left(\mathbf{1}+Y_{h}^{\varepsilon}\right)\left(\mathbf{p}_{0}+\widehat{\mathbf{p}}_{\varepsilon}\right)\right\|_{\mathfrak{G}} \leq \widetilde{\mathcal{C}}_{8} \varepsilon\|\mathbf{F}\|_{\mathfrak{G}}, & 0<\varepsilon \leq 1, \\
\left\|\mathbf{a}_{\varepsilon}-\left(\mathbf{1}+G_{h}^{\varepsilon}\right)\left(\mathbf{a}_{0}+\widehat{\mathbf{a}}_{\varepsilon}\right)\right\|_{\mathfrak{G}} \leq \widetilde{\mathcal{C}}_{9} \varepsilon\|\mathbf{F}\|_{\mathfrak{G}}, & 0<\varepsilon \leq 1 .
\end{aligned}
$$

The constants $\widetilde{\mathcal{C}_{8}}$ and $\widetilde{\mathcal{C}_{9}}$ depend on the norms $\|s\|_{L_{\infty}},\left\|s^{-1}\right\|_{L_{\infty}},\|h\|_{L_{\infty}},\left\|h^{-1}\right\|_{L_{\infty}}$, on $\left\|\nabla \Phi_{\mathbf{e}_{j}}\right\|_{L_{\infty}}, j=1,2,3$, and on the parameters of the lattice $\Gamma$. 
Remark 6.3. 1) Condition (6.16) is satisfied a fortiori if

$$
s \in \widetilde{W}_{\rho}^{1}(\Omega), \quad \rho>3 .
$$

Here the subspace $\widetilde{W}_{\rho}^{1}(\Omega)$ of the Sobolev class $W_{\rho}^{1}(\Omega)$ consists of all functions whose $\Gamma$-periodic extension to $\mathbb{R}^{3}$ belongs to $W_{\rho, \text { loc }}^{1}\left(\mathbb{R}^{3}\right)$. Moreover, (6.21) implies that $\nabla \Phi_{\mathbf{e}_{j}} \in$ $C^{\alpha}(\bar{\Omega})$, where $\alpha=1-3 \rho^{-1}$. (See [LaU, Chapter III, §15].)

2) A slightly weaker sufficient condition on the matrix $s$ ensuring relations (6.16) can be found in $[\mathrm{H}$, Theorem 2.3]. We shall not go into detail here, in order to avoid bulky formulations.

Similarly, $\Pi_{\varepsilon}$ can be eliminated from the last terms in (6.14) and (6.15) if the matrix $h(\mathbf{x})$ is such that

$$
\nabla \Psi_{\mathbf{e}_{j}} \in L_{\infty}, \quad j=1,2,3,
$$

where $\Psi_{\mathbf{e}_{j}}$ is the $\Gamma$-periodic solution of equation (2.23) with $\mathbf{C}=\mathbf{e}_{j}$. Then $Y_{h} \in L_{\infty}$ and $G_{h} \in L_{\infty}$. By (6.12) and (6.13), we have

$$
\begin{aligned}
\left\|Y_{h}^{\varepsilon}\left(I-\Pi_{\varepsilon}\right) \widehat{\mathbf{p}}_{\varepsilon}\right\|_{\mathfrak{G}} & \leq \varepsilon \mathfrak{C}_{1}(s)\left\|h^{-1}\right\|_{L_{\infty}}\left\|s^{-1}\right\|_{L_{\infty}} c_{*}^{-1}\left(1+r_{0}^{-2}\right)^{1 / 2}\left\|Y_{h}\right\|_{L_{\infty}}\|\mathbf{F}\|_{\mathfrak{G}}, \\
\left\|G_{h}^{\varepsilon}\left(I-\Pi_{\varepsilon}\right) \widehat{\mathbf{a}}_{\varepsilon}\right\|_{\mathfrak{G}} & \leq \varepsilon \mathfrak{C}_{1}(s)\left\|s^{-1}\right\|_{L_{\infty}} c_{*}^{-1}\left(1+r_{0}^{-2}\right)^{1 / 2}\left\|G_{h}\right\|_{L_{\infty}}\|\mathbf{F}\|_{\mathfrak{G}} .
\end{aligned}
$$

We put

$$
\begin{aligned}
& \widehat{\mathcal{C}_{8}}=\check{\mathcal{C}}_{8}+\mathfrak{C}_{1}(s)\left\|h^{-1}\right\|_{L_{\infty}}\left\|s^{-1}\right\|_{L_{\infty}} c_{*}^{-1}\left(1+r_{0}^{-2}\right)^{1 / 2}\left\|Y_{h}\right\|_{L_{\infty}}, \\
& \widehat{\mathcal{C}_{9}}=\check{\mathcal{C}}_{9}+\mathfrak{C}_{1}(s)\left\|s^{-1}\right\|_{L_{\infty}} c_{*}^{-1}\left(1+r_{0}^{-2}\right)^{1 / 2}\left\|G_{h}\right\|_{L_{\infty}} .
\end{aligned}
$$

Then relations (6.14), (6.15), (6.23), and (6.24) imply the following statement.

Theorem 6.4. Under the assumptions of Theorem 6.1, suppose, moreover, that condition (6.22) is satisfied. Then

$$
\begin{aligned}
& \left\|\mathbf{p}_{\varepsilon}-\left(\mathbf{1}+Y_{h}^{\varepsilon}\right)\left(\mathbf{p}_{0}+\widehat{\mathbf{p}}_{\varepsilon}\right)\right\|_{\mathfrak{G}} \leq \widehat{\mathcal{C}}_{8} \varepsilon\|\mathbf{F}\|_{\mathfrak{G},}, \quad 0<\varepsilon \leq 1, \\
& \left\|\mathbf{a}_{\varepsilon}-\left(\mathbf{1}+G_{h}^{\varepsilon}\right)\left(\mathbf{a}_{0}+\widehat{\mathbf{a}}_{\varepsilon}\right)\right\|_{\mathfrak{G}} \leq \widehat{\mathcal{C}}_{9} \varepsilon\|\mathbf{F}\|_{\mathfrak{G}}, \quad 0<\varepsilon \leq 1 .
\end{aligned}
$$

The constants $\widehat{\mathcal{C}_{8}}$ and $\widehat{\mathcal{C}_{9}}$ depend on the norms $\|s\|_{L_{\infty}},\left\|s^{-1}\right\|_{L_{\infty}},\|h\|_{L_{\infty}},\left\|h^{-1}\right\|_{L_{\infty}}$, on $\left\|\nabla \Psi_{\mathbf{e}_{j}}\right\|_{L_{\infty}}, j=1,2,3$, and on the parameters of the lattice $\Gamma$.

Remark 6.5. Condition (6.22) is valid a fortiori if

$$
h \in \widetilde{W}_{\rho}^{1}(\Omega), \quad \rho>3 .
$$

6.4. Reformulation of the results. Now we reformulate the results in a form convenient for applications to the Maxwell system. If $\mathbf{f}_{\varepsilon}$ is the solution of problem (5.8), then $\phi_{\varepsilon}=\left(s^{\varepsilon}\right)^{1 / 2} \mathbf{f}_{\varepsilon}$ is the solution of the problem

$$
\operatorname{curl}\left(h^{\varepsilon}\right)^{-1} \operatorname{curl}\left(s^{\varepsilon}\right)^{-1} \phi_{\varepsilon}+\phi_{\varepsilon}=\mathbf{F}, \quad \operatorname{div} \phi_{\varepsilon}=0, \quad \mathbf{F} \in J .
$$

Then $\mathbf{p}_{\varepsilon}$ and $\mathbf{a}_{\varepsilon}$ are related to $\phi_{\varepsilon}$ by the formulas

$$
\begin{aligned}
& \mathbf{p}_{\varepsilon}=\left(h^{\varepsilon}\right)^{-1} \operatorname{curl}\left(s^{\varepsilon}\right)^{-1} \boldsymbol{\phi}_{\varepsilon}, \\
& \mathbf{a}_{\varepsilon}=\operatorname{curl}\left(s^{\varepsilon}\right)^{-1} \boldsymbol{\phi}_{\varepsilon} .
\end{aligned}
$$

Next, if $\mathbf{f}_{0}$ is the solution of (5.33), then $\phi_{0}=\left(s^{0}\right)^{1 / 2} \mathbf{f}_{0}$ is the solution of the problem

$$
\operatorname{curl}\left(h^{0}\right)^{-1} \operatorname{curl}\left(s^{0}\right)^{-1} \phi_{0}+\phi_{0}=\mathbf{F}, \quad \operatorname{div} \phi_{0}=0 .
$$


Then $\mathbf{p}_{0}$ and $\mathbf{a}_{0}$ (see (5.35), (5.38)) are expressed in terms of $\phi_{0}$ by the formulas

$$
\begin{aligned}
& \mathbf{p}_{0}=\left(h^{0}\right)^{-1} \operatorname{curl}\left(s^{0}\right)^{-1} \boldsymbol{\phi}_{0}, \\
& \mathbf{a}_{0}=\operatorname{curl}\left(s^{0}\right)^{-1} \boldsymbol{\phi}_{0} .
\end{aligned}
$$

If $\widehat{\mathbf{f}}_{\varepsilon}$ is the solution of (5.34), then $\widehat{\phi}_{\varepsilon}=\left(s^{0}\right)^{1 / 2} \widehat{\mathbf{f}}_{\varepsilon}$ is the solution of the problem

$$
\operatorname{curl}\left(h^{0}\right)^{-1} \operatorname{curl}\left(s^{0}\right)^{-1} \widehat{\boldsymbol{\phi}}_{\varepsilon}+\widehat{\boldsymbol{\phi}}_{\varepsilon}=\widetilde{\mathcal{P}}\left(s^{0}\right)\left(Y_{s}^{\varepsilon}\right)^{*} \mathbf{F}, \quad \operatorname{div} \widehat{\boldsymbol{\phi}}_{\varepsilon}=0 .
$$

Then $\widehat{\mathbf{p}}_{\varepsilon}$ and $\widehat{\mathbf{a}}_{\varepsilon}$ (see (5.36), (5.39)) can be written as

$$
\begin{aligned}
& \widehat{\mathbf{p}}_{\varepsilon}=\left(h^{0}\right)^{-1} \operatorname{curl}\left(s^{0}\right)^{-1} \widehat{\boldsymbol{\phi}}_{\varepsilon}, \\
& \widehat{\mathbf{a}}_{\varepsilon}=\operatorname{curl}\left(s^{0}\right)^{-1} \widehat{\boldsymbol{\phi}}_{\varepsilon} .
\end{aligned}
$$

Now we formulate the final result. The following theorem combines the results obtained in [Su2 on the basis of estimate (5.3) (see Theorem 10.1 in $\underline{\mathrm{Su} 2}$ ), and estimates (6.8), (6.9), (6.14), and (6.15) proved above.

Theorem 6.6. Let $\phi_{\varepsilon}$ be the solution of problem (6.27), and let $\psi_{\varepsilon}=\left(s^{\varepsilon}\right)^{-1} \phi_{\varepsilon}$. Consider the vector-valued $\mathbf{p}_{\varepsilon}$ and $\mathbf{a}_{\varepsilon}$ defined by (6.28) and (6.29). Denote by $\phi_{0}$ the solution of the "homogenized" problem (6.30), and put $\boldsymbol{\psi}_{0}=\left(s^{0}\right)^{-1} \boldsymbol{\phi}_{0}$. Let $\mathbf{p}_{0}$ and $\mathbf{a}_{0}$ be defined by (6.31) and (6.32). Also, denote by $\widehat{\phi}_{\varepsilon}$ the solution of the "correction" problem (6.33), and put $\widehat{\boldsymbol{\psi}}_{\varepsilon}=\left(s^{0}\right)^{-1} \widehat{\boldsymbol{\phi}}_{\varepsilon}$. Let $\widehat{\mathbf{p}}_{\varepsilon}$ and $\widehat{\mathbf{a}}_{\varepsilon}$ be defined by (6.34) and (6.35). Let $\Pi_{\varepsilon}$ be the $\Psi D O$ (5.4). Consider the matrix $Y_{s}(\mathbf{x})$ with the columns $\nabla \Phi_{\mathbf{e}_{j}}(\mathbf{x}), j=1,2,3$, where $\Phi_{\mathbf{e}_{j}}$ is the periodic solution of equation (2.1) with $\mathbf{C}=\mathbf{e}_{j}$. Put $G_{s}(\mathbf{x})=s(\mathbf{x})\left(\mathbf{1}+Y_{s}(\mathbf{x})\right)\left(s^{0}\right)^{-1}-\mathbf{1}$. Let $Y_{h}(\mathbf{x})$ be the matrix with the columns $\nabla \Psi_{\mathbf{e}_{j}}(\mathbf{x}), j=1,2,3$, where $\Psi_{\mathbf{e}_{j}}$ is the periodic solution of the equation (2.23) with $\mathbf{C}=\mathbf{e}_{j}$. Put $G_{h}(\mathbf{x})=h(\mathbf{x})\left(\mathbf{1}+Y_{h}(\mathbf{x})\right)\left(h^{0}\right)^{-1}-\mathbf{1}$. Then for $0<\varepsilon \leq 1$ we have

$$
\begin{aligned}
& \left\|\phi_{\varepsilon}-\left(\mathbf{1}+G_{s}^{\varepsilon}\right)\left(\phi_{0}+\Pi_{\varepsilon} \widehat{\phi}_{\varepsilon}\right)\right\|_{\mathfrak{G}} \leq \mathcal{C}_{10} \varepsilon\|\mathbf{F}\|_{\mathfrak{G}}, \quad 0<\varepsilon \leq 1, \\
& \left\|\phi_{\varepsilon}-\left(\mathbf{1}+G_{s}^{\varepsilon}\right)\left(\phi_{0}+\widehat{\phi}_{\varepsilon}\right)\right\|_{\mathfrak{G}} \leq \check{\mathcal{C}}_{10} \varepsilon\|\mathbf{F}\|_{\mathfrak{G}}, \quad 0<\varepsilon \leq 1, \\
& \left\|\boldsymbol{\psi}_{\varepsilon}-\left(\mathbf{1}+Y_{s}^{\varepsilon}\right)\left(\boldsymbol{\psi}_{0}+\Pi_{\varepsilon} \widehat{\boldsymbol{\psi}}_{\varepsilon}\right)\right\|_{\mathfrak{G}} \leq \mathcal{C}_{11} \varepsilon\|\mathbf{F}\|_{\mathfrak{G}}, \quad 0<\varepsilon \leq 1, \\
& \left\|\boldsymbol{\psi}_{\varepsilon}-\left(\mathbf{1}+Y_{s}^{\varepsilon}\right)\left(\boldsymbol{\psi}_{0}+\widehat{\boldsymbol{\psi}}_{\varepsilon}\right)\right\|_{\mathfrak{G}} \leq \check{\mathcal{C}}_{11} \varepsilon\|\mathbf{F}\|_{\mathfrak{G}}, \quad 0<\varepsilon \leq 1, \\
& \left\|\mathbf{p}_{\varepsilon}-\left(\mathbf{1}+Y_{h}^{\varepsilon}\right)\left(\mathbf{p}_{0}+\Pi_{\varepsilon} \widehat{\mathbf{p}}_{\varepsilon}\right)\right\|_{\mathfrak{G}} \leq \mathcal{C}_{8} \varepsilon\|\mathbf{F}\|_{\mathfrak{G}}, \quad 0<\varepsilon \leq 1, \\
& \left\|\mathbf{p}_{\varepsilon}-\left(\mathbf{p}_{0}+Y_{h}^{\varepsilon} \mathbf{p}_{0}+\widehat{\mathbf{p}}_{\varepsilon}+Y_{h}^{\varepsilon} \Pi_{\varepsilon} \widehat{\mathbf{p}}_{\varepsilon}\right)\right\|_{\mathfrak{G}} \leq \check{\mathcal{C}}_{8} \varepsilon\|\mathbf{F}\|_{\mathfrak{G},}, \quad 0<\varepsilon \leq 1, \\
& \left\|\mathbf{a}_{\varepsilon}-\left(\mathbf{1}+G_{h}^{\varepsilon}\right)\left(\mathbf{a}_{0}+\Pi_{\varepsilon} \widehat{\mathbf{a}}_{\varepsilon}\right)\right\|_{\mathfrak{G}} \leq \mathcal{C}_{9} \varepsilon\|\mathbf{F}\|_{\mathfrak{G},}, \quad 0<\varepsilon \leq 1, \\
& \left\|\mathbf{a}_{\varepsilon}-\left(\mathbf{a}_{0}+G_{h}^{\varepsilon} \mathbf{a}_{0}+\widehat{\mathbf{a}}_{\varepsilon}+G_{h}^{\varepsilon} \Pi_{\varepsilon} \widehat{\mathbf{a}}_{\varepsilon}\right)\right\|_{\mathfrak{G}} \leq \check{\mathcal{C}}_{9} \varepsilon\|\mathbf{F}\|_{\mathfrak{G}}, \quad 0<\varepsilon \leq 1 .
\end{aligned}
$$

The constants $\mathcal{C}_{8}, \check{\mathcal{C}}_{8}, \mathcal{C}_{9}, \check{\mathcal{C}}_{9}, \mathcal{C}_{10}, \check{\mathcal{C}}_{10}, \mathcal{C}_{11}$, and $\check{\mathcal{C}}_{11}$ depend only on $\|h\|_{L_{\infty}},\left\|h^{-1}\right\|_{L_{\infty}}$, $\|s\|_{L_{\infty}},\left\|s^{-1}\right\|_{L_{\infty}}$, and the parameters of the lattice $\Gamma$.

Here we have deliberately presented two versions of approximation for each field. The approximations (6.36), (6.38), (6.40), and (6.42) involve the smoothing operator applied to the solution of the "correction" problem. For the fields $\phi_{\varepsilon}$ and $\boldsymbol{\psi}_{\varepsilon}$, we can get rid of the smoothing operator completely; this is illustrated by the approximations (6.37) and (6.39). At the same time, for the fields $\mathbf{p}_{\varepsilon}$ and $\mathbf{a}_{\varepsilon}$, the smoothing operator can be eliminated only partly; this is demonstrated by the approximations (6.41) and (6.43).

Now we reformulate Theorems 6.2 and 6.4 in a form convenient for further applications.

Theorem 6.7. Under the assumptions of Theorem 6.6,

$1^{\circ}$. If condition (6.16) is satisfied, then estimates (6.19) and (6.20) are true;.

$2^{\circ}$. If condition (6.22) is satisfied, then estimates (6.25) and (6.26) are true. 


\section{§7. The stationary Maxwell system}

7.1. Statement of the problem. Below $\mathbf{u}$ and $\mathbf{v}$ stand for the electric field strength and the magnetic field strength. We assume that the magnetic permeability $\mu(\mathbf{x})$ and the electric permittivity $\eta(\mathbf{x})$ are $\Gamma$-periodic matrix-valued functions having real entries and satisfying conditions of the form (1.1). The electric and magnetic displacement vectors $\mathbf{w}$ and $\mathbf{z}$ are related to $\mathbf{u}$ and $\mathbf{v}$ by the formulas $\mathbf{w}=\eta(\mathbf{x}) \mathbf{u}$ and $\mathbf{z}=\mu(\mathbf{x}) \mathbf{v}$. Recall the notation $\mathfrak{G}=L_{2}\left(\mathbb{R}^{3} ; \mathbb{C}^{3}\right)$ and $J=\{\mathbf{f} \in \mathfrak{G}: \operatorname{div} \mathbf{f}=0\}$. We denote by $\mathfrak{G}\left(\eta^{-1}\right)=L_{2}\left(\mathbb{R}^{3} ; \mathbb{C}^{3} ; \eta^{-1}\right)$ the "weighted" space with the inner product $\left(\mathbf{f}_{1}, \mathbf{f}_{2}\right)_{\mathfrak{G}\left(\eta^{-1}\right)}=$ $\left(\eta^{-1} \mathbf{f}_{1}, \mathbf{f}_{2}\right)_{\mathfrak{G}}$. The space $\mathfrak{G}\left(\mu^{-1}\right)$ is defined similarly. The set $J$ is a closed subspace in $\mathfrak{G}$, as well as in the weighted spaces $\mathfrak{G}\left(\eta^{-1}\right)$ and $\mathfrak{G}\left(\mu^{-1}\right)$.

Written in terms of displacement vectors, the Maxwell operator $\mathcal{M}=\mathcal{M}(\eta, \mu)$ acts in the space $J \oplus J$ and is given by the formula

$$
\mathcal{M}=\mathcal{M}(\eta, \mu)=\left(\begin{array}{cc}
0 & i \operatorname{curl} \mu^{-1} \\
-i \operatorname{curl} \eta^{-1} & 0
\end{array}\right)
$$

on the domain

$$
\operatorname{Dom} \mathcal{M}(\eta, \mu)=\left\{(\mathbf{w}, \mathbf{z}): \mathbf{w} \in J, \mathbf{z} \in J, \operatorname{curl} \eta^{-1} \mathbf{w} \in \mathfrak{G}, \operatorname{curl} \mu^{-1} \mathbf{z} \in \mathfrak{G}\right\} .
$$

The operator $\mathcal{M}$ is closed, but not selfadjoint in $J \oplus J$ (relative to the standard inner product in $\mathfrak{G} \oplus \mathfrak{G})$. However, $\mathcal{M}$ is selfadjoint in the space $J \oplus J$ viewed as a subspace of $\mathfrak{G}\left(\eta^{-1}\right) \oplus \mathfrak{G}\left(\mu^{-1}\right)$. The point $\lambda=i$ is a regular point of $\mathcal{M}$.

Recall the notation $\varphi^{\varepsilon}(\mathbf{x})=\varphi\left(\varepsilon^{-1} \mathbf{x}\right)$ (for a given $\Gamma$-periodic measurable function $\varphi$ ). We introduce the family of operators $\mathcal{M}_{\varepsilon}=\mathcal{M}\left(\eta^{\varepsilon}, \mu^{\varepsilon}\right), \varepsilon>0$, acting in $J \oplus J$. Here Dom $\mathcal{M}_{\varepsilon}$ depends on $\varepsilon$. Our goal is to study the behavior of the resolvent $\left(\mathcal{M}_{\varepsilon}-i I\right)^{-1}$ as $\varepsilon \rightarrow 0$. Consider the equation

$$
\left(\mathcal{M}_{\varepsilon}-i I\right)\left(\begin{array}{c}
\mathbf{w}_{\varepsilon} \\
\mathbf{z}_{\varepsilon}
\end{array}\right)=\left(\begin{array}{l}
\mathbf{q} \\
\mathbf{r}
\end{array}\right), \quad \mathbf{q}, \mathbf{r} \in J
$$

The corresponding strengths are given by the relations

$$
\mathbf{u}_{\varepsilon}=\left(\eta^{\varepsilon}\right)^{-1} \mathbf{w}_{\varepsilon}, \quad \mathbf{v}_{\varepsilon}=\left(\mu^{\varepsilon}\right)^{-1} \mathbf{z}_{\varepsilon} .
$$

In detail, (7.2) takes the form

$$
\begin{array}{r}
i \operatorname{curl}\left(\mu^{\varepsilon}\right)^{-1} \mathbf{z}_{\varepsilon}-i \mathbf{w}_{\varepsilon}=\mathbf{q}, \\
-i \operatorname{curl}\left(\eta^{\varepsilon}\right)^{-1} \mathbf{w}_{\varepsilon}-i \mathbf{z}_{\varepsilon}=\mathbf{r}, \\
\operatorname{div} \mathbf{w}_{\varepsilon}=0, \quad \operatorname{div} \mathbf{z}_{\varepsilon}=0 .
\end{array}
$$

We represent the solutions as sums (cf. [BSu2, Chapter 7; Su1,2; BSu6]): $\mathbf{w}_{\varepsilon}=\mathbf{w}_{\varepsilon}^{(q)}+$ $\mathbf{w}_{\varepsilon}^{(r)}, \mathbf{z}_{\varepsilon}=\mathbf{z}_{\varepsilon}^{(q)}+\mathbf{z}_{\varepsilon}^{(r)}$, where the pair of vectors $\mathbf{w}_{\varepsilon}^{(q)}, \mathbf{z}_{\varepsilon}^{(q)}$ is the solution of system (7.4) with $\mathbf{r}=0$, and the pair of vectors $\mathbf{w}_{\varepsilon}^{(r)}, \mathbf{z}_{\varepsilon}^{(r)}$ is the solution of system (7.4) with $\mathbf{q}=0$. Accordingly, the fields $\mathbf{u}_{\varepsilon}, \mathbf{v}_{\varepsilon}$ are also represented as sums.

7.2. The effective operator. Let $\eta^{0}$ be the effective matrix for the operator $-\operatorname{div} \eta \nabla$ (cf. (2.12)), and let $\mu^{0}$ be the effective matrix for the operator $-\operatorname{div} \mu \nabla$. Let $\mathcal{M}^{0}=$ $\mathcal{M}\left(\eta^{0}, \mu^{0}\right)$ be the effective Maxwell operator. We introduce the "homogenized" Maxwell system

$$
\left(\mathcal{M}^{0}-i I\right)\left(\begin{array}{c}
\mathbf{w}_{0} \\
\mathbf{z}_{0}
\end{array}\right)=\left(\begin{array}{l}
\mathbf{q} \\
\mathbf{r}
\end{array}\right), \quad \mathbf{q}, \mathbf{r} \in J
$$

The corresponfing fields $\mathbf{u}_{0}, \mathbf{v}_{0}$ are related to $\mathbf{w}_{0}, \mathbf{z}_{0}$ by the formulas

$$
\mathbf{u}_{0}=\left(\eta^{0}\right)^{-1} \mathbf{w}_{0}, \quad \mathbf{v}_{0}=\left(\mu^{0}\right)^{-1} \mathbf{z}_{0} .
$$


The solutions of system (7.5) are also represented as sums of two terms: $\mathbf{w}_{0}=\mathbf{w}_{0}^{(q)}+\mathbf{w}_{0}^{(r)}$, $\mathbf{z}_{0}=\mathbf{z}_{0}^{(q)}+\mathbf{z}_{0}^{(r)}$, and similarly for $\mathbf{u}_{0}, \mathbf{v}_{0}$.

7.3. The case where $\mathbf{q}=0$. If $\mathbf{q}=0$, system (7.4) takes the form

$$
\begin{gathered}
\mathbf{w}_{\varepsilon}^{(r)}=\operatorname{curl}\left(\mu^{\varepsilon}\right)^{-1} \mathbf{z}_{\varepsilon}^{(r)}, \\
\operatorname{curl}\left(\eta^{\varepsilon}\right)^{-1} \mathbf{w}_{\varepsilon}^{(r)}+\mathbf{z}_{\varepsilon}^{(r)}=i \mathbf{r}, \\
\operatorname{div} \mathbf{w}_{\varepsilon}^{(r)}=0, \quad \operatorname{div} \mathbf{z}_{\varepsilon}^{(r)}=0 .
\end{gathered}
$$

The strengths are given by the formulas

$$
\mathbf{u}_{\varepsilon}^{(r)}=\left(\eta^{\varepsilon}\right)^{-1} \mathbf{w}_{\varepsilon}^{(r)}, \quad \mathbf{v}_{\varepsilon}^{(r)}=\left(\mu^{\varepsilon}\right)^{-1} \mathbf{z}_{\varepsilon}^{(r)} .
$$

From (7.7) it is clear that $\mathbf{z}_{\varepsilon}^{(r)}$ is the solution of the problem

$$
\operatorname{curl}\left(\eta^{\varepsilon}\right)^{-1} \operatorname{curl}\left(\mu^{\varepsilon}\right)^{-1} \mathbf{z}_{\varepsilon}^{(r)}+\mathbf{z}_{\varepsilon}^{(r)}=i \mathbf{r}, \quad \operatorname{div} \mathbf{z}_{\varepsilon}^{(r)}=0, \quad \mathbf{r} \in J
$$

Herewith,

$$
\begin{aligned}
\mathbf{w}_{\varepsilon}^{(r)} & =\operatorname{curl}\left(\mu^{\varepsilon}\right)^{-1} \mathbf{z}_{\varepsilon}^{(r)}, \\
\mathbf{u}_{\varepsilon}^{(r)} & =\left(\eta^{\varepsilon}\right)^{-1} \operatorname{curl}\left(\mu^{\varepsilon}\right)^{-1} \mathbf{z}_{\varepsilon}^{(r)} .
\end{aligned}
$$

Now the conditions of Theorem 6.6 are satisfied. Comparing (7.8) with (6.27), (7.9) with (6.29), and (7.10) with (6.28), we see that $h=\eta, s=\mu, \mathbf{F}=i \mathbf{r}, \phi_{\varepsilon}=\mathbf{z}_{\varepsilon}^{(r)}, \boldsymbol{\psi}_{\varepsilon}=\mathbf{v}_{\varepsilon}^{(r)}$, $\mathbf{p}_{\varepsilon}=\mathbf{u}_{\varepsilon}^{(r)}$, and $\mathbf{a}_{\varepsilon}=\mathbf{w}_{\varepsilon}^{(r)}$.

Next, from (7.5) with $\mathbf{q}=0$ it is clear that the "homogenized" magnetic displacement vector $\mathbf{z}_{0}^{(r)}$ is the solution of the problem

$$
\operatorname{curl}\left(\eta^{0}\right)^{-1} \operatorname{curl}\left(\mu^{0}\right)^{-1} \mathbf{z}_{0}^{(r)}+\mathbf{z}_{0}^{(r)}=i \mathbf{r}, \quad \operatorname{div} \mathbf{z}_{0}^{(r)}=0, \quad \mathbf{r} \in J,
$$

which corresponds to problem (6.30). Now in the conditions of Theorem 6.6 we must put $\phi_{0}=\mathbf{z}_{0}^{(r)}, \boldsymbol{\psi}_{0}=\mathbf{v}_{0}^{(r)}, \mathbf{p}_{0}=\mathbf{u}_{0}^{(r)}$, and $\mathbf{a}_{0}=\mathbf{w}_{0}^{(r)}$.

We introduce the "correction" Maxwell system

$$
\left(\mathcal{M}^{0}-i I\right)\left(\begin{array}{c}
\widehat{\mathbf{w}}_{\varepsilon}^{(r)} \\
\widehat{\mathbf{z}}_{\varepsilon}^{(r)}
\end{array}\right)=\left(\begin{array}{c}
0 \\
\mathbf{r}_{\varepsilon}
\end{array}\right), \quad \mathbf{r}_{\varepsilon}=\widetilde{\mathcal{P}}\left(\mu^{0}\right)\left(Y_{\mu}^{\varepsilon}\right)^{*} \mathbf{r},
$$

and put

$$
\widehat{\mathbf{u}}_{\varepsilon}^{(r)}=\left(\eta^{0}\right)^{-1} \widehat{\mathbf{w}}_{\varepsilon}^{(r)}, \quad \widehat{\mathbf{v}}_{\varepsilon}^{(r)}=\left(\mu^{0}\right)^{-1} \widehat{\mathbf{z}}_{\varepsilon}^{(r)} .
$$

Then $\widehat{\mathbf{z}}_{\varepsilon}^{(r)}$ is the solution of the problem

$$
\operatorname{curl}\left(\eta^{0}\right)^{-1} \operatorname{curl}\left(\mu^{0}\right)^{-1} \widehat{\mathbf{z}}_{\varepsilon}^{(r)}+\widehat{\mathbf{z}}_{\varepsilon}^{(r)}=i \mathbf{r}_{\varepsilon}, \quad \operatorname{div} \widehat{\mathbf{z}}_{\varepsilon}^{(r)}=0,
$$

which corresponds to problem (6.33). Now in the conditions of Theorem 6.6 we have $\widehat{\boldsymbol{\phi}}_{\varepsilon}=\widehat{\mathbf{z}}_{\varepsilon}^{(r)}, \widehat{\boldsymbol{\psi}}_{\varepsilon}=\widehat{\mathbf{v}}_{\varepsilon}^{(r)}$. System (7.11) implies that

$$
\begin{aligned}
& \widehat{\mathbf{w}}_{\varepsilon}^{(r)}=\operatorname{curl}\left(\mu^{0}\right)^{-1} \widehat{\mathbf{z}}_{\varepsilon}^{(r)}, \\
& \widehat{\mathbf{u}}_{\varepsilon}^{(r)}=\left(\eta^{0}\right)^{-1} \operatorname{curl}\left(\mu^{0}\right)^{-1} \widehat{\mathbf{z}}_{\varepsilon}^{(r)} ;
\end{aligned}
$$

i.e., now $\widehat{\mathbf{p}}_{\varepsilon}=\widehat{\mathbf{u}}_{\varepsilon}^{(r)}$ and $\widehat{\mathbf{a}}_{\varepsilon}=\widehat{\mathbf{w}}_{\varepsilon}^{(r)}($ see $(6.34),(6.35))$. 
T. A. SUSLINA

Applying Theorem 6.6, we obtain the following approximations for the magnetic fields $\mathbf{v}_{\varepsilon}^{(r)}$ and $\mathbf{z}_{\varepsilon}^{(r)}$ :

$$
\begin{array}{rlrl}
\left\|\mathbf{v}_{\varepsilon}^{(r)}-\left(\mathbf{1}+Y_{\mu}^{\varepsilon}\right)\left(\mathbf{v}_{0}^{(r)}+\Pi_{\varepsilon} \widehat{\mathbf{v}}_{\varepsilon}^{(r)}\right)\right\|_{\mathfrak{G}} & \leq \mathcal{C}_{11}^{(r)} \varepsilon\|\mathbf{r}\|_{\mathfrak{G},} & & 0<\varepsilon \leq 1, \\
\left\|\mathbf{v}_{\varepsilon}^{(r)}-\left(\mathbf{1}+Y_{\mu}^{\varepsilon}\right)\left(\mathbf{v}_{0}^{(r)}+\widehat{\mathbf{v}}_{\varepsilon}^{(r)}\right)\right\|_{\mathfrak{G}} & \leq \check{\mathcal{C}}_{11}^{(r)} \varepsilon\|\mathbf{r}\|_{\mathfrak{G},} & 0<\varepsilon \leq 1, \\
\left\|\mathbf{z}_{\varepsilon}^{(r)}-\left(\mathbf{1}+G_{\mu}^{\varepsilon}\right)\left(\mathbf{z}_{0}^{(r)}+\Pi_{\varepsilon} \widehat{\mathbf{z}}_{\varepsilon}^{(r)}\right)\right\|_{\mathfrak{G}} \leq \mathcal{C}_{10}^{(r)} \varepsilon\|\mathbf{r}\|_{\mathfrak{G},} & 0<\varepsilon \leq 1, \\
\left\|\mathbf{z}_{\varepsilon}^{(r)}-\left(\mathbf{1}+G_{\mu}^{\varepsilon}\right)\left(\mathbf{z}_{0}^{(r)}+\widehat{\mathbf{z}}_{\varepsilon}^{(r)}\right)\right\|_{\mathfrak{G}} \leq \check{\mathcal{C}}_{10}^{(r)} \varepsilon\|\mathbf{r}\|_{\mathfrak{G},} & 0<\varepsilon \leq 1 .
\end{array}
$$

Here and below the constants with the upper index $(r)$ are the realizations of the constants with the same numbers for $h=\eta$ and $s=\mu$. The approximations (7.12)-(7.15) were obtained before in $\mathrm{Su} 2$, Theorem 12.1].

Theorem 6.6 implies the following approximations for the electric fields $\mathbf{u}_{\varepsilon}^{(r)}$ and $\mathbf{w}_{\varepsilon}^{(r)}$ :

$$
\begin{aligned}
\left\|\mathbf{u}_{\varepsilon}^{(r)}-\left(\mathbf{1}+Y_{\eta}^{\varepsilon}\right)\left(\mathbf{u}_{0}^{(r)}+\Pi_{\varepsilon} \widehat{\mathbf{u}}_{\varepsilon}^{(r)}\right)\right\|_{\mathfrak{G}} \leq \mathcal{C}_{8}^{(r)} \varepsilon\|\mathbf{r}\|_{\mathfrak{G},} & 0<\varepsilon \leq 1, \\
\left\|\mathbf{u}_{\varepsilon}^{(r)}-\left(\mathbf{u}_{0}^{(r)}+Y_{\eta}^{\varepsilon} \mathbf{u}_{0}^{(r)}+\widehat{\mathbf{u}}_{\varepsilon}^{(r)}+Y_{\eta}^{\varepsilon} \Pi_{\varepsilon} \widehat{\mathbf{u}}_{\varepsilon}^{(r)}\right)\right\|_{\mathfrak{G}} \leq \check{\mathcal{C}}_{8}^{(r)} \varepsilon\|\mathbf{r}\|_{\mathfrak{G},} & 0<\varepsilon \leq 1, \\
\left\|\mathbf{w}_{\varepsilon}^{(r)}-\left(\mathbf{1}+G_{\eta}^{\varepsilon}\right)\left(\mathbf{w}_{0}^{(r)}+\Pi_{\varepsilon} \widehat{\mathbf{w}}_{\varepsilon}^{(r)}\right)\right\|_{\mathfrak{G}} \leq \mathcal{C}_{9}^{(r)} \varepsilon\|\mathbf{r}\|_{\mathfrak{G},}, & 0<\varepsilon \leq 1, \\
\left\|\mathbf{w}_{\varepsilon}^{(r)}-\left(\mathbf{w}_{0}^{(r)}+G_{\eta}^{\varepsilon} \mathbf{w}_{0}^{(r)}+\widehat{\mathbf{w}}_{\varepsilon}^{(r)}+G_{\eta}^{\varepsilon} \Pi_{\varepsilon} \widehat{\mathbf{w}}_{\varepsilon}^{(r)}\right)\right\|_{\mathfrak{G}} \leq \check{\mathcal{C}}_{9}^{(r)} \varepsilon\|\mathbf{r}\|_{\mathfrak{G},}, & 0<\varepsilon \leq 1 .
\end{aligned}
$$

Theorem $6.7\left(1^{\circ}\right)$ shows that if

$$
\nabla \Phi_{\mathbf{e}_{j}} \in L_{\infty}, \quad j=1,2,3,
$$

where $\Phi_{\mathbf{e}_{j}}$ is the $\Gamma$-periodic solution of equation (2.1) with $s=\mu$ and $\mathbf{C}=\mathbf{e}_{j}$, then

$$
\begin{array}{cl}
\left\|\mathbf{u}_{\varepsilon}^{(r)}-\left(\mathbf{1}+Y_{\eta}^{\varepsilon}\right)\left(\mathbf{u}_{0}^{(r)}+\widehat{\mathbf{u}}_{\varepsilon}^{(r)}\right)\right\|_{\mathfrak{G}} \leq \widetilde{\mathcal{C}}_{8}^{(r)} \varepsilon\|\mathbf{r}\|_{\mathfrak{G},}, & 0<\varepsilon \leq 1, \\
\left\|\mathbf{w}_{\varepsilon}^{(r)}-\left(\mathbf{1}+G_{\eta}^{\varepsilon}\right)\left(\mathbf{w}_{0}^{(r)}+\widehat{\mathbf{w}}_{\varepsilon}^{(r)}\right)\right\|_{\mathfrak{G}} \leq \widetilde{\mathcal{C}}_{9}^{(r)} \varepsilon\|\mathbf{r}\|_{\mathfrak{G},}, & 0<\varepsilon \leq 1 .
\end{array}
$$

The constants $\widetilde{\mathcal{C}}_{8}^{(r)}, \widetilde{\mathcal{C}}_{9}^{(r)}$ realize the constants $\widetilde{\mathcal{C}}_{8}, \widetilde{\mathcal{C}}_{9}$ for $h=\eta$ and $s=\mu$.

Similarly, Theorem $6.7\left(2^{\circ}\right)$ shows that if

$$
\nabla \Psi_{\mathbf{e}_{j}} \in L_{\infty}, \quad j=1,2,3,
$$

where $\Psi_{\mathbf{e}_{j}}$ is the $\Gamma$-periodic solution of equation (2.23) with $h=\eta$ and $\mathbf{C}=\mathbf{e}_{j}$, then

$$
\begin{array}{cl}
\left\|\mathbf{u}_{\varepsilon}^{(r)}-\left(\mathbf{1}+Y_{\eta}^{\varepsilon}\right)\left(\mathbf{u}_{0}^{(r)}+\widehat{\mathbf{u}}_{\varepsilon}^{(r)}\right)\right\|_{\mathfrak{G}} \leq \widehat{\mathcal{C}}_{8}^{(r)} \varepsilon\|\mathbf{r}\|_{\mathfrak{G},}, & 0<\varepsilon \leq 1, \\
\left\|\mathbf{w}_{\varepsilon}^{(r)}-\left(\mathbf{1}+G_{\eta}^{\varepsilon}\right)\left(\mathbf{w}_{0}^{(r)}+\widehat{\mathbf{w}}_{\varepsilon}^{(r)}\right)\right\|_{\mathfrak{G}} \leq \widehat{\mathcal{C}}_{9}^{(r)} \varepsilon\|\mathbf{r}\|_{\mathfrak{G},}, \quad 0<\varepsilon \leq 1 .
\end{array}
$$

The constants $\widehat{\mathcal{C}}_{8}^{(r)}, \widehat{\mathcal{C}}_{9}^{(r)}$ realize the constants $\widehat{\mathcal{C}_{8}}, \widehat{\mathcal{C}}_{9}$ for $h=\eta$ and $s=\mu$.

7.4. The case where $\mathbf{r}=0$. If $\mathbf{r}=0$, then system (7.4) for the displacement vectors $\mathbf{w}_{\varepsilon}^{(q)}$ and $\mathbf{z}_{\varepsilon}^{(q)}$ takes the form

$$
\begin{gathered}
\mathbf{z}_{\varepsilon}^{(q)}=-\operatorname{curl}\left(\eta^{\varepsilon}\right)^{-1} \mathbf{w}_{\varepsilon}^{(q)}, \\
-\operatorname{curl}\left(\mu^{\varepsilon}\right)^{-1} \mathbf{z}_{\varepsilon}^{(q)}+\mathbf{w}_{\varepsilon}^{(q)}=i \mathbf{q}, \\
\operatorname{div} \mathbf{w}_{\varepsilon}^{(q)}=0, \quad \operatorname{div} \mathbf{z}_{\varepsilon}^{(q)}=0 .
\end{gathered}
$$

The strengths are written as

$$
\mathbf{u}_{\varepsilon}^{(q)}=\left(\eta^{\varepsilon}\right)^{-1} \mathbf{w}_{\varepsilon}^{(q)}, \quad \mathbf{v}_{\varepsilon}^{(q)}=\left(\mu^{\varepsilon}\right)^{-1} \mathbf{z}_{\varepsilon}^{(q)} .
$$

From (7.26) we see that $\mathbf{w}_{\varepsilon}^{(q)}$ is the solution of the problem

$$
\operatorname{curl}\left(\mu^{\varepsilon}\right)^{-1} \operatorname{curl}\left(\eta^{\varepsilon}\right)^{-1} \mathbf{w}_{\varepsilon}^{(q)}+\mathbf{w}_{\varepsilon}^{(q)}=i \mathbf{q}, \quad \operatorname{div} \mathbf{w}_{\varepsilon}^{(q)}=0 .
$$


Herewith,

$$
\begin{aligned}
& \mathbf{z}_{\varepsilon}^{(q)}=-\operatorname{curl}\left(\eta^{\varepsilon}\right)^{-1} \mathbf{w}_{\varepsilon}^{(q)}, \\
& \mathbf{v}_{\varepsilon}^{(q)}=-\left(\mu^{\varepsilon}\right)^{-1} \operatorname{curl}\left(\eta^{\varepsilon}\right)^{-1} \mathbf{w}_{\varepsilon}^{(q)} .
\end{aligned}
$$

Again, the conditions of Theorem 6.6 are satisfied, but now we have $h=\mu, s=\eta$, and $\mathbf{F}=i \mathbf{q}$. Then $\boldsymbol{\phi}_{\varepsilon}=\mathbf{w}_{\varepsilon}^{(q)}, \boldsymbol{\psi}_{\varepsilon}=\mathbf{u}_{\varepsilon}^{(q)}, \mathbf{p}_{\varepsilon}=-\mathbf{v}_{\varepsilon}^{(q)}$, and $\mathbf{a}_{\varepsilon}=-\mathbf{z}_{\varepsilon}^{(q)}$.

System (7.5) with $\mathbf{r}=0$ shows that $\mathbf{w}_{0}^{(q)}$ is the solution of the problem

$$
\operatorname{curl}\left(\mu^{0}\right)^{-1} \operatorname{curl}\left(\eta^{0}\right)^{-1} \mathbf{w}_{0}^{(q)}+\mathbf{w}_{0}^{(q)}=i \mathbf{q}, \quad \operatorname{div} \mathbf{w}_{0}^{(q)}=0,
$$

which corresponds to problem (6.30). Now, in the conditions of Theorem 6.6, we put $\phi_{0}=\mathbf{w}_{0}^{(q)}, \boldsymbol{\psi}_{0}=\mathbf{u}_{0}^{(q)}, \mathbf{p}_{0}=-\mathbf{v}_{0}^{(q)}$, and $\mathbf{a}_{0}=-\mathbf{z}_{0}^{(q)}$.

We introduce the "correction" system

$$
\left(\mathcal{M}^{0}-i I\right)\left(\begin{array}{c}
\widehat{\mathbf{w}}_{\varepsilon}^{(q)} \\
\widehat{\mathbf{z}}_{\varepsilon}^{(q)}
\end{array}\right)=\left(\begin{array}{c}
\mathbf{q}_{\varepsilon} \\
0
\end{array}\right), \quad \mathbf{q}_{\varepsilon}=\widetilde{\mathcal{P}}\left(\eta^{0}\right)\left(Y_{\eta}^{\varepsilon}\right)^{*} \mathbf{q}
$$

Then $\widehat{\mathbf{w}}_{\varepsilon}^{(q)}$ is the solution of the problem

$$
\operatorname{curl}\left(\mu^{0}\right)^{-1} \operatorname{curl}\left(\eta^{0}\right)^{-1} \widehat{\mathbf{w}}_{\varepsilon}^{(q)}+\widehat{\mathbf{w}}_{\varepsilon}^{(q)}=i \mathbf{q}_{\varepsilon}, \quad \operatorname{div} \widehat{\mathbf{w}}_{\varepsilon}^{(q)}=0,
$$

which corresponds to problem (6.33). We put

$$
\widehat{\mathbf{u}}_{\varepsilon}^{(q)}=\left(\eta^{0}\right)^{-1} \widehat{\mathbf{w}}_{\varepsilon}^{(q)}, \quad \widehat{\mathbf{v}}_{\varepsilon}^{(q)}=\left(\mu^{0}\right)^{-1} \widehat{\mathbf{z}}_{\varepsilon}^{(q)} .
$$

System (7.27) implies

$$
\begin{aligned}
& \widehat{\mathbf{z}}_{\varepsilon}^{(q)}=-\operatorname{curl}\left(\eta^{0}\right)^{-1} \widehat{\mathbf{w}}_{\varepsilon}^{(q)}, \\
& \widehat{\mathbf{v}}_{\varepsilon}^{(q)}=-\left(\mu^{0}\right)^{-1} \operatorname{curl}\left(\eta^{0}\right)^{-1} \widehat{\mathbf{w}}_{\varepsilon}^{(q)} .
\end{aligned}
$$

Thus, now in the conditions of Theorem 6.6 , we put $\widehat{\boldsymbol{\phi}}_{\varepsilon}=\widehat{\mathbf{w}}_{\varepsilon}^{(q)}, \widehat{\boldsymbol{\psi}}_{\varepsilon}=\widehat{\mathbf{u}}_{\varepsilon}^{(q)}, \widehat{\mathbf{p}}_{\varepsilon}=-\widehat{\mathbf{v}}_{\varepsilon}^{(q)}$, and $\widehat{\mathbf{a}}_{\varepsilon}=-\widehat{\mathbf{z}}_{\varepsilon}^{(q)}$.

Applying Theorem 6.6, we obtain the following approximations for the electric fields $\mathbf{u}_{\varepsilon}^{(q)}$ and $\mathbf{w}_{\varepsilon}^{(q)}$ :

$$
\begin{aligned}
\left\|\mathbf{u}_{\varepsilon}^{(q)}-\left(\mathbf{1}+Y_{\eta}^{\varepsilon}\right)\left(\mathbf{u}_{0}^{(q)}+\Pi_{\varepsilon} \widehat{\mathbf{u}}_{\varepsilon}^{(q)}\right)\right\|_{\mathfrak{G}} \leq \mathcal{C}_{11}^{(q)} \varepsilon\|\mathbf{q}\|_{\mathfrak{G},}, & 0<\varepsilon \leq 1, \\
\left\|\mathbf{u}_{\varepsilon}^{(q)}-\left(\mathbf{1}+Y_{\eta}^{\varepsilon}\right)\left(\mathbf{u}_{0}^{(q)}+\widehat{\mathbf{u}}_{\varepsilon}^{(q)}\right)\right\|_{\mathfrak{G}} \leq \check{\mathcal{C}}_{11}^{(q)} \varepsilon\|\mathbf{q}\|_{\mathfrak{G},}, & 0<\varepsilon \leq 1, \\
\left\|\mathbf{w}_{\varepsilon}^{(q)}-\left(\mathbf{1}+G_{\eta}^{\varepsilon}\right)\left(\mathbf{w}_{0}^{(q)}+\Pi_{\varepsilon} \widehat{\mathbf{w}}_{\varepsilon}^{(q)}\right)\right\|_{\mathfrak{G}} \leq \mathcal{C}_{10}^{(q)} \varepsilon\|\mathbf{q}\|_{\mathfrak{G}}, & 0<\varepsilon \leq 1, \\
\left\|\mathbf{w}_{\varepsilon}^{(q)}-\left(\mathbf{1}+G_{\eta}^{\varepsilon}\right)\left(\mathbf{w}_{0}^{(q)}+\widehat{\mathbf{w}}_{\varepsilon}^{(q)}\right)\right\|_{\mathfrak{G}} \leq \check{\mathcal{C}}_{10}^{(q)} \varepsilon\|\mathbf{q}\|_{\mathfrak{G},}, & 0<\varepsilon \leq 1 .
\end{aligned}
$$

Here and below the constants with upper index $(q)$ realize the constants with the same numbers for $h=\mu$ and $s=\eta$. The approximations (7.28)-(7.31) were obtained before in Su2, Theorem 12.2].

Theorem 6.6 also implies the following approximations for the magnetic fields $\mathbf{v}_{\varepsilon}^{(q)}$ and $\mathbf{z}_{\varepsilon}^{(q)}$ :

$$
\begin{aligned}
& \left\|\mathbf{v}_{\varepsilon}^{(q)}-\left(\mathbf{1}+Y_{\mu}^{\varepsilon}\right)\left(\mathbf{v}_{0}^{(q)}+\Pi_{\varepsilon} \widehat{\mathbf{v}}_{\varepsilon}^{(q)}\right)\right\|_{\mathfrak{G}} \leq \mathcal{C}_{8}^{(q)} \varepsilon\|\mathbf{q}\|_{\mathfrak{G},}, \quad 0<\varepsilon \leq 1, \\
& \left\|\mathbf{v}_{\varepsilon}^{(q)}-\left(\mathbf{v}_{0}^{(q)}+Y_{\mu}^{\varepsilon} \mathbf{v}_{0}^{(q)}+\widehat{\mathbf{v}}_{\varepsilon}^{(q)}+Y_{\mu}^{\varepsilon} \Pi_{\varepsilon} \widehat{\mathbf{v}}_{\varepsilon}^{(q)}\right)\right\|_{\mathfrak{G}} \leq \check{\mathcal{C}}_{8}^{(q)} \varepsilon\|\mathbf{q}\|_{\mathfrak{G},}, \quad 0<\varepsilon \leq 1, \\
& \left\|\mathbf{z}_{\varepsilon}^{(q)}-\left(\mathbf{1}+G_{\mu}^{\varepsilon}\right)\left(\mathbf{z}_{0}^{(q)}+\Pi_{\varepsilon} \widehat{\mathbf{z}}_{\varepsilon}^{(q)}\right)\right\|_{\mathfrak{G}} \leq \mathcal{C}_{9}^{(q)} \varepsilon\|\mathbf{q}\|_{\mathfrak{G},}, \quad 0<\varepsilon \leq 1, \\
& \left\|\mathbf{z}_{\varepsilon}^{(q)}-\left(\mathbf{z}_{0}^{(q)}+G_{\mu}^{\varepsilon} \mathbf{z}_{0}^{(q)}+\widehat{\mathbf{z}}_{\varepsilon}^{(q)}+G_{\mu}^{\varepsilon} \Pi_{\varepsilon} \widehat{\mathbf{z}}_{\varepsilon}^{(q)}\right)\right\|_{\mathfrak{G}} \leq \check{\mathcal{C}}_{9}^{(q)} \varepsilon\|\mathbf{q}\|_{\mathfrak{G}}, \quad 0<\varepsilon \leq 1 .
\end{aligned}
$$


Theorem $6.7\left(1^{\circ}\right)$ shows that, under condition (7.23), we have

$$
\begin{aligned}
& \left\|\mathbf{v}_{\varepsilon}^{(q)}-\left(\mathbf{1}+Y_{\mu}^{\varepsilon}\right)\left(\mathbf{v}_{0}^{(q)}+\widehat{\mathbf{v}}_{\varepsilon}^{(q)}\right)\right\|_{\mathfrak{G}} \leq \widetilde{\mathcal{C}}_{8}^{(q)} \varepsilon\|\mathbf{q}\|_{\mathfrak{G}}, \quad 0<\varepsilon \leq 1, \\
& \left\|\mathbf{z}_{\varepsilon}^{(q)}-\left(\mathbf{1}+G_{\mu}^{\varepsilon}\right)\left(\mathbf{z}_{0}^{(q)}+\widehat{\mathbf{z}}_{\varepsilon}^{(q)}\right)\right\|_{\mathfrak{G}} \leq \widetilde{\mathcal{C}}_{9}^{(q)} \varepsilon\|\mathbf{q}\|_{\mathfrak{G}}, \quad 0<\varepsilon \leq 1,
\end{aligned}
$$

where the constants $\widetilde{\mathcal{C}}_{8}^{(q)}, \widetilde{\mathcal{C}}_{9}^{(q)}$ realize the constants $\widetilde{\mathcal{C}}_{8}, \widetilde{\mathcal{C}}_{9}$ for $h=\mu$ and $s=\eta$.

Similarly, Theorem $6.7\left(2^{\circ}\right)$ shows that, under condition (7.20), we have

$$
\begin{gathered}
\left\|\mathbf{v}_{\varepsilon}^{(q)}-\left(\mathbf{1}+Y_{\mu}^{\varepsilon}\right)\left(\mathbf{v}_{0}^{(q)}+\widehat{\mathbf{v}}_{\varepsilon}^{(q)}\right)\right\|_{\mathfrak{G}} \leq \widehat{\mathcal{C}}_{8}^{(q)} \varepsilon\|\mathbf{q}\|_{\mathfrak{G},}, \quad 0<\varepsilon \leq 1, \\
\left\|\mathbf{z}_{\varepsilon}^{(q)}-\left(\mathbf{1}+G_{\mu}^{\varepsilon}\right)\left(\mathbf{z}_{0}^{(q)}+\widehat{\mathbf{z}}_{\varepsilon}^{(q)}\right)\right\|_{\mathfrak{G}} \leq \widehat{\mathcal{C}}_{9}^{(q)} \varepsilon\|\mathbf{q}\|_{\mathfrak{G},}, \quad 0<\varepsilon \leq 1,
\end{gathered}
$$

where the constants $\widehat{\mathcal{C}}_{8}^{(q)}, \widehat{\mathcal{C}}_{9}^{(q)}$ realize the constants $\widehat{\mathcal{C}_{8}}, \widehat{\mathcal{C}_{9}}$ for $h=\mu$ and $s=\eta$.

7.5. Summarized results. We sum estimates (7.16) and (7.28), (7.18) and (7.30), (7.12) and (7.32), and (7.14) and (7.34). For convenience of formulation, we introduce the "total" correction Maxwell system

$$
\left(\mathcal{M}^{0}-i I\right)\left(\begin{array}{c}
\widehat{\mathbf{w}}_{\varepsilon} \\
\widehat{\mathbf{z}}_{\varepsilon}
\end{array}\right)=\left(\begin{array}{c}
\mathbf{q}_{\varepsilon} \\
\mathbf{r}_{\varepsilon}
\end{array}\right), \quad \mathbf{q}_{\varepsilon}=\widetilde{\mathcal{P}}\left(\eta^{0}\right)\left(Y_{\eta}^{\varepsilon}\right)^{*} \mathbf{q}, \quad \mathbf{r}_{\varepsilon}=\widetilde{\mathcal{P}}\left(\mu^{0}\right)\left(Y_{\mu}^{\varepsilon}\right)^{*} \mathbf{r} .
$$

The corresponding strengths are given by the relations

$$
\widehat{\mathbf{u}}_{\varepsilon}=\left(\eta^{0}\right)^{-1} \widehat{\mathbf{w}}_{\varepsilon}, \quad \widehat{\mathbf{v}}_{\varepsilon}=\left(\mu^{0}\right)^{-1} \widehat{\mathbf{z}}_{\varepsilon} .
$$

We arrive at the following result.

Theorem 7.1. Let $\left(\mathbf{w}_{\varepsilon}, \mathbf{z}_{\varepsilon}\right)$ be the solution of system (7.2), and let the fields $\mathbf{u}_{\varepsilon}, \mathbf{v}_{\varepsilon}$ be defined by (7.3). Let $\left(\mathbf{w}_{0}, \mathbf{z}_{0}\right)$ be the solution of the homogenized system (7.5), and let the fields $\mathbf{u}_{0}, \mathbf{v}_{0}$ be defined by (7.6). Let $\left(\widehat{\mathbf{w}}_{\varepsilon}, \widehat{\mathbf{z}}_{\varepsilon}\right)$ be the solution of the correction system (7.40), and let the fields $\widehat{\mathbf{u}}_{\varepsilon}, \widehat{\mathbf{v}}_{\varepsilon}$ be defined by (7.41). Denote by $\Pi_{\varepsilon}$ the $\Psi D O$ (5.4). Suppose that the matrix-valued functions $Y_{\eta}, G_{\eta}$ are defined in terms of the matrix $\eta$, and $Y_{\mu}, G_{\mu}$ are defined in terms of $\mu$, in accordance with the rule described in Theorem 6.6. Then for $0<\varepsilon \leq 1$, the fields $\mathbf{u}_{\varepsilon}, \mathbf{w}_{\varepsilon}, \mathbf{v}_{\varepsilon}$, and $\mathbf{z}_{\varepsilon}$ satisfy the following approximations in the $\mathfrak{G}$-norm with the error estimate of order $\varepsilon$ :

$$
\begin{aligned}
\left\|\mathbf{u}_{\varepsilon}-\left(\mathbf{1}+Y_{\eta}^{\varepsilon}\right)\left(\mathbf{u}_{0}+\Pi_{\varepsilon} \widehat{\mathbf{u}}_{\varepsilon}\right)\right\|_{\mathfrak{G}} & \leq \varepsilon\left(\mathcal{C}_{11}^{(q)}\|\mathbf{q}\|_{\mathfrak{G}}+\mathcal{C}_{8}^{(r)}\|\mathbf{r}\|_{\mathfrak{G}}\right), \\
\left\|\mathbf{w}_{\varepsilon}-\left(\mathbf{1}+G_{\eta}^{\varepsilon}\right)\left(\mathbf{w}_{0}+\Pi_{\varepsilon} \widehat{\mathbf{w}}_{\varepsilon}\right)\right\|_{\mathfrak{G}} & \leq \varepsilon\left(\mathcal{C}_{10}^{(q)}\|\mathbf{q}\|_{\mathfrak{G}}+\mathcal{C}_{9}^{(r)}\|\mathbf{r}\|_{\mathfrak{G}}\right), \\
\left\|\mathbf{v}_{\varepsilon}-\left(\mathbf{1}+Y_{\mu}^{\varepsilon}\right)\left(\mathbf{v}_{0}+\Pi_{\varepsilon} \widehat{\mathbf{v}}_{\varepsilon}\right)\right\|_{\mathfrak{G}} & \leq \varepsilon\left(\mathcal{C}_{8}^{(q)}\|\mathbf{q}\|_{\mathfrak{G}}+\mathcal{C}_{11}^{(r)}\|\mathbf{r}\|_{\mathfrak{G}}\right), \\
\left\|\mathbf{z}_{\varepsilon}-\left(\mathbf{1}+G_{\mu}^{\varepsilon}\right)\left(\mathbf{z}_{0}+\Pi_{\varepsilon} \widehat{\mathbf{z}}_{\varepsilon}\right)\right\|_{\mathfrak{G}} & \leq \varepsilon\left(\mathcal{C}_{9}^{(q)}\|\mathbf{q}\|_{\mathfrak{G}}+\mathcal{C}_{10}^{(r)}\|\mathbf{r}\|_{\mathfrak{G}}\right) .
\end{aligned}
$$

The constants in (7.42)-(7.45) depend only on $\|\eta\|_{L_{\infty}},\left\|\eta^{-1}\right\|_{L_{\infty}},\|\mu\|_{L_{\infty}},\left\|\mu^{-1}\right\|_{L_{\infty}}$, and the parameters of the lattice $\Gamma$.

We see that approximations for separate terms sum successfully. The approximations (7.42)-(7.45) are of the same structure. For instance, the approximation for the field $\mathbf{u}_{\varepsilon}$ is given by the sum of four terms:

$$
\mathbf{u}_{0}+Y_{\eta}^{\varepsilon} \mathbf{u}_{0}+\Pi_{\varepsilon} \widehat{\mathbf{u}}_{\varepsilon}+Y_{\eta}^{\varepsilon} \Pi_{\varepsilon} \widehat{\mathbf{u}}_{\varepsilon},
$$

where the first term does not depend on $\varepsilon$ and is the "homogenized" field, while the other terms involve rapidly oscillating factors with zero mean. These terms tend weakly to zero in $\mathfrak{G}$ as $\varepsilon \rightarrow 0$; they are correctors of zero order.

The approximations (7.42)-(7.45) involve the smoothing operator $\Pi_{\varepsilon}$ applied to the solution of the correction problem. As we have seen, the smoothing operator $\Pi_{\varepsilon}$ can be 
eliminated from some terms. In this sense, the result of Theorem 7.1 is not optimal. We distinguish Theorem 7.1 because of compactness of formulation.

Summing estimates (7.17) and (7.29), (7.19) and (7.31), (7.13) and (7.33), and (7.15) and (7.35), we obtain a more optimal (in the sense of elimination of the smoothing operator), but at the same time more bulky result. This leads to the following theorem.

Theorem 7.2. Suppose that the conditions of Theorem 7.1 are satisfied. Let $\left(\widehat{\mathbf{w}}_{\varepsilon}^{(q)}, \widehat{\mathbf{z}}_{\varepsilon}^{(q)}\right)$ be the solution of system (7.27), and let $\widehat{\mathbf{u}}_{\varepsilon}^{(q)}=\left(\eta^{0}\right)^{-1} \widehat{\mathbf{w}}_{\varepsilon}^{(q)}$ and $\widehat{\mathbf{v}}_{\varepsilon}^{(q)}=\left(\mu^{0}\right)^{-1} \widehat{\mathbf{z}}_{\varepsilon}^{(q)}$. Let $\left(\widehat{\mathbf{w}}_{\varepsilon}^{(r)}, \widehat{\mathbf{z}}_{\varepsilon}^{(r)}\right)$ be the solution of system $(7.11)$, and let $\widehat{\mathbf{u}}_{\varepsilon}^{(r)}=\left(\eta^{0}\right)^{-1} \widehat{\mathbf{w}}_{\varepsilon}^{(r)}$ and $\widehat{\mathbf{v}}_{\varepsilon}^{(r)}=$ $\left(\mu^{0}\right)^{-1} \widehat{\mathbf{z}}_{\varepsilon}^{(r)}$. Then for $0<\varepsilon \leq 1$ the fields $\mathbf{u}_{\varepsilon}, \mathbf{w}_{\varepsilon}, \mathbf{v}_{\varepsilon}, \mathbf{z}_{\varepsilon}$ satisfy the following approximations in the $\mathfrak{G}$-norm with error estimate of order $\varepsilon$ :

$$
\begin{aligned}
& \left\|\mathbf{u}_{\varepsilon}-\left(\left(\mathbf{1}+Y_{\eta}^{\varepsilon}\right) \mathbf{u}_{0}+\widehat{\mathbf{u}}_{\varepsilon}+Y_{\eta}^{\varepsilon}\left(\widehat{\mathbf{u}}_{\varepsilon}^{(q)}+\Pi_{\varepsilon} \widehat{\mathbf{u}}_{\varepsilon}^{(r)}\right)\right)\right\|_{\mathfrak{G}} \leq \varepsilon\left(\check{\mathcal{C}}_{11}^{(q)}\|\mathbf{q}\|_{\mathfrak{G}}+\check{\mathcal{C}}_{8}^{(r)}\|\mathbf{r}\|_{\mathfrak{G}}\right), \\
& \left\|\mathbf{w}_{\varepsilon}-\left(\left(\mathbf{1}+G_{\eta}^{\varepsilon}\right) \mathbf{w}_{0}+\widehat{\mathbf{w}}_{\varepsilon}+G_{\eta}^{\varepsilon}\left(\widehat{\mathbf{w}}_{\varepsilon}^{(q)}+\Pi_{\varepsilon} \widehat{\mathbf{w}}_{\varepsilon}^{(r)}\right)\right)\right\|_{\mathfrak{G}} \leq \varepsilon\left(\check{\mathcal{C}}_{10}^{(q)}\|\mathbf{q}\|_{\mathfrak{G}}+\check{\mathcal{C}}_{9}^{(r)}\|\mathbf{r}\|_{\mathfrak{G}}\right), \\
& \left\|\mathbf{v}_{\varepsilon}-\left(\left(\mathbf{1}+Y_{\mu}^{\varepsilon}\right) \mathbf{v}_{0}+\widehat{\mathbf{v}}_{\varepsilon}+Y_{\mu}^{\varepsilon}\left(\Pi_{\varepsilon} \widehat{\mathbf{v}}_{\varepsilon}^{(q)}+\widehat{\mathbf{v}}_{\varepsilon}^{(r)}\right)\right)\right\|_{\mathfrak{G}} \leq \varepsilon\left(\check{\mathcal{C}}_{8}^{(q)}\|\mathbf{q}\|_{\mathfrak{G}}+\check{\mathcal{C}}_{11}^{(r)}\|\mathbf{r}\|_{\mathfrak{G}}\right), \\
& \left\|\mathbf{z}_{\varepsilon}-\left(\left(\mathbf{1}+G_{\mu}^{\varepsilon}\right) \mathbf{z}_{0}+\widehat{\mathbf{z}}_{\varepsilon}+G_{\mu}^{\varepsilon}\left(\Pi_{\varepsilon} \widehat{\mathbf{z}}_{\varepsilon}^{(q)}+\widehat{\mathbf{z}}_{\varepsilon}^{(r)}\right)\right)\right\|_{\mathfrak{G}} \leq \varepsilon\left(\check{\mathcal{C}}_{9}^{(q)}\|\mathbf{q}\|_{\mathfrak{G}}+\check{\mathcal{C}}_{10}^{(r)}\|\mathbf{r}\|_{\mathfrak{G}}\right) .
\end{aligned}
$$

The constants in (7.46)-(7.49) depend only on $\|\eta\|_{L_{\infty}},\left\|\eta^{-1}\right\|_{L_{\infty}},\|\mu\|_{L_{\infty}},\left\|\mu^{-1}\right\|_{L_{\infty}}$, and the parameters of the lattice $\Gamma$.

If the additional condition (7.20) is satisfied, it is possible to eliminate the smoothing operator completely. For this, we sum estimates (7.29) and (7.21), (7.31) and (7.22), (7.38) and (7.13), (7.39) and (7.15). Similarly, under condition (7.23), we sum estimates (7.29) and (7.24), (7.31) and (7.25), (7.36) and (7.13), and (7.37) and (7.15). We arrive at the following result.

Theorem 7.3. Suppose that the assumptions of Theorem 7.1 are fulfilled.

$1^{\circ}$. If condition (7.20) is satisfied, then for $0<\varepsilon \leq 1$ we have

$$
\begin{aligned}
\left\|\mathbf{u}_{\varepsilon}-\left(\mathbf{1}+Y_{\eta}^{\varepsilon}\right)\left(\mathbf{u}_{0}+\widehat{\mathbf{u}}_{\varepsilon}\right)\right\|_{\mathfrak{G}} & \leq \varepsilon\left(\check{\mathcal{C}}_{11}^{(q)}\|\mathbf{q}\|_{\mathfrak{G}}+\widetilde{\mathcal{C}}_{8}^{(r)}\|\mathbf{r}\|_{\mathfrak{G}}\right), \\
\left\|\mathbf{w}_{\varepsilon}-\left(\mathbf{1}+G_{\eta}^{\varepsilon}\right)\left(\mathbf{w}_{0}+\widehat{\mathbf{w}}_{\varepsilon}\right)\right\|_{\mathfrak{G}} & \leq \varepsilon\left(\check{\mathcal{C}}_{10}^{(q)}\|\mathbf{q}\|_{\mathfrak{G}}+\widetilde{\mathcal{C}}_{9}^{(r)}\|\mathbf{r}\|_{\mathfrak{G}}\right), \\
\left\|\mathbf{v}_{\varepsilon}-\left(\mathbf{1}+Y_{\mu}^{\varepsilon}\right)\left(\mathbf{v}_{0}+\widehat{\mathbf{v}}_{\varepsilon}\right)\right\|_{\mathfrak{G}} & \leq \varepsilon\left(\widehat{\mathcal{C}}_{8}^{(q)}\|\mathbf{q}\|_{\mathfrak{G}}+\check{\mathcal{C}}_{11}^{(r)}\|\mathbf{r}\|_{\mathfrak{G}}\right), \\
\left\|\mathbf{z}_{\varepsilon}-\left(\mathbf{1}+G_{\mu}^{\varepsilon}\right)\left(\mathbf{z}_{0}+\widehat{\mathbf{z}}_{\varepsilon}\right)\right\|_{\mathfrak{G}} & \leq \varepsilon\left(\widehat{\mathcal{C}}_{9}^{(q)}\|\mathbf{q}\|_{\mathfrak{G}}+\check{\mathcal{C}}_{10}^{(r)}\|\mathbf{r}\|_{\mathfrak{G}}\right) .
\end{aligned}
$$

The constants $\check{\mathcal{C}}_{10}^{(q)}, \check{\mathcal{C}}_{11}^{(q)}, \check{\mathcal{C}}_{10}^{(r)}, \check{\mathcal{C}}_{11}^{(r)}$ depend only on $\|\eta\|_{L_{\infty}},\left\|\eta^{-1}\right\|_{L_{\infty}},\|\mu\|_{L_{\infty}},\left\|\mu^{-1}\right\|_{L_{\infty}}$, and the parameters of the lattice $\Gamma$, and the constants $\widetilde{\mathcal{C}}_{8}^{(\infty)}, \widetilde{\mathcal{C}}_{9}^{(r)}, \widehat{\mathcal{C}}_{8}^{(q)}, \widehat{\mathcal{C}}_{9}^{(q)}$ depend on the same parameters and also on the norms $\left\|\nabla \Phi_{\mathbf{e}_{j}}\right\|_{L_{\infty}}, j=1,2,3$.

$2^{\circ}$. If condition (7.23) is satisfied, then for $0<\varepsilon \leq 1$ we have

$$
\begin{aligned}
\left\|\mathbf{u}_{\varepsilon}-\left(\mathbf{1}+Y_{\eta}^{\varepsilon}\right)\left(\mathbf{u}_{0}+\widehat{\mathbf{u}}_{\varepsilon}\right)\right\|_{\mathfrak{G}} & \leq \varepsilon\left(\check{\mathcal{C}}_{11}^{(q)}\|\mathbf{q}\|_{\mathfrak{G}}+\widehat{\mathcal{C}}_{8}^{(r)}\|\mathbf{r}\|_{\mathfrak{G}}\right), \\
\left\|\mathbf{w}_{\varepsilon}-\left(\mathbf{1}+G_{\eta}^{\varepsilon}\right)\left(\mathbf{w}_{0}+\widehat{\mathbf{w}}_{\varepsilon}\right)\right\|_{\mathfrak{G}} & \leq \varepsilon\left(\check{\mathcal{C}}_{10}^{(q)}\|\mathbf{q}\|_{\mathfrak{G}}+\widehat{\mathcal{C}}_{9}^{(r)}\|\mathbf{r}\|_{\mathfrak{G}}\right), \\
\left\|\mathbf{v}_{\varepsilon}-\left(\mathbf{1}+Y_{\mu}^{\varepsilon}\right)\left(\mathbf{v}_{0}+\widehat{\mathbf{v}}_{\varepsilon}\right)\right\|_{\mathfrak{G}} & \leq \varepsilon\left(\widetilde{\mathcal{C}}_{8}^{(q)}\|\mathbf{q}\|_{\mathfrak{G}}+\check{\mathcal{C}}_{11}^{(r)}\|\mathbf{r}\|_{\mathfrak{G}}\right), \\
\left\|\mathbf{z}_{\varepsilon}-\left(\mathbf{1}+G_{\mu}^{\varepsilon}\right)\left(\mathbf{z}_{0}+\widehat{\mathbf{z}}_{\varepsilon}\right)\right\|_{\mathfrak{G}} & \leq \varepsilon\left(\widetilde{\mathcal{C}}_{9}^{(q)}\|\mathbf{q}\|_{\mathfrak{G}}+\check{\mathcal{C}}_{10}^{(r)}\|\mathbf{r}\|_{\mathfrak{G}}\right) .
\end{aligned}
$$


The constants $\check{\mathcal{C}}_{10}^{(q)}, \check{\mathcal{C}}_{11}^{(q)}, \check{\mathcal{C}}_{10}^{(r)}, \check{\mathcal{C}}_{11}^{(r)}$ depend only on $\|\eta\|_{L_{\infty}},\left\|\eta^{-1}\right\|_{L_{\infty}},\|\mu\|_{L_{\infty}},\left\|\mu^{-1}\right\|_{L_{\infty}}$, and the parameters of the lattice $\Gamma$, and the constants $\widehat{\mathcal{C}}_{8}^{(r)}, \widehat{\mathcal{C}}_{9}^{(r)}, \widetilde{\mathcal{C}}_{8}^{(q)}, \widetilde{\mathcal{C}}_{9}^{(q)}$ depend on the same parameters and also on the norms $\left\|\nabla \Psi_{\mathbf{e}_{j}}\right\|_{L_{\infty}}, j=1,2,3$.

Remark 7.4. Under the assumptions of Theorem $7.3\left(1^{\circ}\right)$, condition (7.20) can be replaced by the condition $\mu \in \widetilde{W}_{\rho}^{1}(\Omega), \rho>3$. Similarly, under the assumptions of Theorem $7.3\left(2^{\circ}\right)$, condition (7.23) can be replaced by the condition $\eta \in \widetilde{W}_{\rho}^{1}(\Omega), \rho>3$. (See Remarks 6.3 and 6.5.)

7.6. Special cases. We consider the case where

$$
\eta^{0}=\bar{\eta}=|\Omega|^{-1} \int_{\Omega} \eta(\mathbf{x}) d \mathbf{x} .
$$

This condition is equivalent to the fact that the columns of the matrix $\eta(\mathbf{x})$ are divergencefree vectors (see [ZhKO] or [BSu2, Chapter 3, Proposition 1.6]). Let $\Psi_{\mathbf{e}_{j}}$ be the $\Gamma$-periodic solution of equation (2.23) with $h=\eta$ and $\mathbf{C}=\mathbf{e}_{j}$. If condition (7.54) is satisfied, then $\Psi_{\mathbf{e}_{j}}=0, j=1,2,3$. Hence,

$$
Y_{\eta}(\mathbf{x})=0, \quad G_{\eta}(\mathbf{x})=\eta(\mathbf{x})\left(\eta^{0}\right)^{-1}-\mathbf{1} .
$$

Note that now we have $G_{\eta} \in L_{\infty}$. The right-hand side of the correction system (7.27) is equal to zero: $\mathbf{q}_{\varepsilon}=0$. Then

$$
\begin{aligned}
& \widehat{\mathbf{u}}_{\varepsilon}^{(q)}=0, \quad \widehat{\mathbf{w}}_{\varepsilon}^{(q)}=0, \quad \widehat{\mathbf{v}}_{\varepsilon}^{(q)}=0, \quad \widehat{\mathbf{z}}_{\varepsilon}^{(q)}=0, \\
& \widehat{\mathbf{u}}_{\varepsilon}=\widehat{\mathbf{u}}_{\varepsilon}^{(r)}, \quad \widehat{\mathbf{w}}_{\varepsilon}=\widehat{\mathbf{w}}_{\varepsilon}^{(r)}, \quad \widehat{\mathbf{v}}_{\varepsilon}=\widehat{\mathbf{v}}_{\varepsilon}^{(r)}, \quad \widehat{\mathbf{z}}_{\varepsilon}=\widehat{\mathbf{z}}_{\varepsilon}^{(r)} .
\end{aligned}
$$

By (7.55) and (7.56), relation (7.46) implies

$$
\left\|\mathbf{u}_{\varepsilon}-\left(\mathbf{u}_{0}+\widehat{\mathbf{u}}_{\varepsilon}^{(r)}\right)\right\|_{\mathfrak{G}} \leq \varepsilon\left(\check{\mathcal{C}}_{11}^{(q)}\|\mathbf{q}\|_{\mathfrak{G}}+\check{\mathcal{C}}_{8}^{(r)}\|\mathbf{r}\|_{\mathfrak{G}}\right), \quad 0<\varepsilon \leq 1 .
$$

Since $\Psi_{\mathbf{e}_{j}}=0, j=1,2,3$, condition (7.23) is satisfied. Then, by Theorem $7.3\left(2^{\circ}\right)$, estimate $(7.51)$ is true. Now this estimate takes the form

$$
\left\|\mathbf{w}_{\varepsilon}-\eta^{\varepsilon}\left(\eta^{0}\right)^{-1}\left(\mathbf{w}_{0}+\widehat{\mathbf{w}}_{\varepsilon}^{(r)}\right)\right\|_{\mathfrak{G}} \leq \varepsilon\left(\check{\mathcal{C}}_{10}^{(q)}\|\mathbf{q}\|_{\mathfrak{G}}+\widehat{\mathcal{C}}_{9}^{(r)}\|\mathbf{r}\|_{\mathfrak{G}}\right), \quad 0<\varepsilon \leq 1
$$

Next, combining (7.48) and (7.49) with (7.56), we obtain

$$
\begin{aligned}
\left\|\mathbf{v}_{\varepsilon}-\left(\mathbf{1}+Y_{\mu}^{\varepsilon}\right)\left(\mathbf{v}_{0}+\widehat{\mathbf{v}}_{\varepsilon}^{(r)}\right)\right\|_{\mathfrak{G}} \leq \varepsilon\left(\check{\mathcal{C}}_{8}^{(q)}\|\mathbf{q}\|_{\mathfrak{G}}+\check{\mathcal{C}}_{11}^{(r)}\|\mathbf{r}\|_{\mathfrak{G}}\right), & 0<\varepsilon \leq 1, \\
\left\|\mathbf{z}_{\varepsilon}-\left(\mathbf{1}+G_{\mu}^{\varepsilon}\right)\left(\mathbf{z}_{0}+\widehat{\mathbf{z}}_{\varepsilon}^{(r)}\right)\right\|_{\mathfrak{G}} \leq \varepsilon\left(\check{\mathcal{C}}_{9}^{(q)}\|\mathbf{q}\|_{\mathfrak{G}}+\check{\mathcal{C}}_{10}^{(r)}\|\mathbf{r}\|_{\mathfrak{G}}\right), & 0<\varepsilon \leq 1 .
\end{aligned}
$$

Note that all approximations (7.57)-(7.60) do not involve the smoothing operator $\Pi_{\varepsilon}$. We have proved the following statement.

Proposition 7.5. Under the condition (7.54), estimates (7.57)-(7.60) are true. The constants in these estimates depend only on $\|\eta\|_{L_{\infty}},\left\|\eta^{-1}\right\|_{L_{\infty}},\|\mu\|_{L_{\infty}},\left\|\mu^{-1}\right\|_{L_{\infty}}$, and the parameters of the lattice $\Gamma$.

The case where

$$
\mu^{0}=\bar{\mu}=|\Omega|^{-1} \int_{\Omega} \mu(\mathbf{x}) d \mathbf{x}
$$

is analyzed similarly. We state the corresponding result. 
Proposition 7.6. Under the condition (7.61), for $0<\varepsilon \leq 1$ we have

$$
\begin{aligned}
\left\|\mathbf{u}_{\varepsilon}-\left(\mathbf{1}+Y_{\eta}^{\varepsilon}\right)\left(\mathbf{u}_{0}+\widehat{\mathbf{u}}_{\varepsilon}^{(q)}\right)\right\|_{\mathfrak{G}} & \leq \varepsilon\left(\check{\mathcal{C}}_{11}^{(q)}\|\mathbf{q}\|_{\mathfrak{G}}+\check{\mathcal{C}}_{8}^{(r)}\|\mathbf{r}\|_{\mathfrak{G}}\right), \\
\left\|\mathbf{w}_{\varepsilon}-\left(\mathbf{1}+G_{\eta}^{\varepsilon}\right)\left(\mathbf{w}_{0}+\widehat{\mathbf{w}}_{\varepsilon}^{(q)}\right)\right\|_{\mathfrak{G}} & \leq \varepsilon\left(\check{\mathcal{C}}_{10}^{(q)}\|\mathbf{q}\|_{\mathfrak{G}}+\check{\mathcal{C}}_{9}^{(r)}\|\mathbf{r}\|_{\mathfrak{G}}\right), \\
\left\|\mathbf{v}_{\varepsilon}-\left(\mathbf{v}_{0}+\widehat{\mathbf{v}}_{\varepsilon}^{(q)}\right)\right\|_{\mathfrak{G}} & \leq \varepsilon\left(\check{\mathcal{C}}_{8}^{(q)}\|\mathbf{q}\|_{\mathfrak{G}}+\check{\mathcal{C}}_{11}^{(r)}\|\mathbf{r}\|_{\mathfrak{G}}\right), \\
\left\|\mathbf{z}_{\varepsilon}-\mu^{\varepsilon}\left(\mu^{0}\right)^{-1}\left(\mathbf{z}_{0}+\widehat{\mathbf{z}}_{\varepsilon}^{(q)}\right)\right\|_{\mathfrak{G}} & \leq \varepsilon\left(\widehat{\mathcal{C}}_{9}^{(q)}\|\mathbf{q}\|_{\mathfrak{G}}+\check{\mathcal{C}}_{10}^{(r)}\|\mathbf{r}\|_{\mathfrak{G}}\right) .
\end{aligned}
$$

The constants in (7.62)-(7.65) depend only on $\|\eta\|_{L_{\infty}},\left\|\eta^{-1}\right\|_{L_{\infty}},\|\mu\|_{L_{\infty}},\left\|\mu^{-1}\right\|_{L_{\infty}}$, and the parameters of the lattice $\Gamma$.

Now we consider the case where

$$
\eta^{0}=\underline{\eta}=\left(|\Omega|^{-1} \int_{\Omega} \eta(\mathbf{x})^{-1} d \mathbf{x}\right)^{-1} .
$$

This condition is equivalent to the fact that the columns of the matrix $\eta(\mathbf{x})^{-1}$ are potential vectors (see [ZhKO] or [BSu2, Chapter 3, Proposition 1.7]). Then $\widetilde{\eta}(\mathbf{x})=\eta^{0}=\underline{\eta}$ (see Remark 3.5 in [BSu4]). In this case we have

$$
G_{\eta}(\mathbf{x})=0, \quad Y_{\eta}(\mathbf{x})=\eta(\mathbf{x})^{-1} \eta^{0}-\mathbf{1} .
$$

Therefore, $Y_{\eta} \in L_{\infty}$; i.e., condition (7.23) is satisfied. By (7.47) and (7.67), we have

$$
\left\|\mathbf{w}_{\varepsilon}-\left(\mathbf{w}_{0}+\widehat{\mathbf{w}}_{\varepsilon}\right)\right\|_{\mathfrak{G}} \leq \varepsilon\left(\check{\mathcal{C}}_{10}^{(q)}\|\mathbf{q}\|_{\mathfrak{G}}+\check{\mathcal{C}}_{9}^{(r)}\|\mathbf{r}\|_{\mathfrak{G}}\right) .
$$

Theorem $7.3\left(2^{\circ}\right)$ shows that estimate (7.50) is fulfilled. Now this estimate takes the form

$$
\left\|\mathbf{u}_{\varepsilon}-\left(\eta^{\varepsilon}\right)^{-1} \eta^{0}\left(\mathbf{u}_{0}+\widehat{\mathbf{u}}_{\varepsilon}\right)\right\|_{\mathfrak{G}} \leq \varepsilon\left(\check{\mathcal{C}}_{11}^{(q)}\|\mathbf{q}\|_{\mathfrak{G}}+\widehat{\mathcal{C}}_{8}^{(r)}\|\mathbf{r}\|_{\mathfrak{G}}\right) .
$$

Estimates (7.52) and (7.53) are also fulfilled:

$$
\begin{aligned}
\left\|\mathbf{v}_{\varepsilon}-\left(\mathbf{1}+Y_{\mu}^{\varepsilon}\right)\left(\mathbf{v}_{0}+\widehat{\mathbf{v}}_{\varepsilon}\right)\right\|_{\mathfrak{G}} & \leq \varepsilon\left(\widetilde{\mathcal{C}}_{8}^{(q)}\|\mathbf{q}\|_{\mathfrak{G}}+\check{\mathcal{C}}_{11}^{(r)}\|\mathbf{r}\|_{\mathfrak{G}}\right), \\
\left\|\mathbf{z}_{\varepsilon}-\left(\mathbf{1}+G_{\mu}^{\varepsilon}\right)\left(\mathbf{z}_{0}+\widehat{\mathbf{z}}_{\varepsilon}\right)\right\|_{\mathfrak{G}} & \leq \varepsilon\left(\widetilde{\mathcal{C}}_{9}^{(q)}\|\mathbf{q}\|_{\mathfrak{G}}+\check{\mathcal{C}}_{10}^{(r)}\|\mathbf{r}\|_{\mathfrak{G}}\right) .
\end{aligned}
$$

Estimates (7.68)-(7.71) do not involve the smoothing operator $\Pi_{\varepsilon}$. We summarize the results.

Proposition 7.7. Under condition (7.66), estimates (7.68)-(7.71) are true. The constants in these estimates depend only on $\|\eta\|_{L_{\infty}},\left\|\eta^{-1}\right\|_{L_{\infty}},\|\mu\|_{L_{\infty}},\left\|\mu^{-1}\right\|_{L_{\infty}}$, and the parameters of the lattice $\Gamma$.

The case where

$$
\mu^{0}=\underline{\mu}=\left(|\Omega|^{-1} \int_{\Omega} \mu(\mathbf{x})^{-1} d \mathbf{x}\right)^{-1}
$$

is analyzed similarly. We formulate the corresponding result.

Proposition 7.8. Under condition (7.72), for $0<\varepsilon \leq 1$ we have

$$
\begin{aligned}
&\left\|\mathbf{u}_{\varepsilon}-\left(\mathbf{1}+Y_{\eta}^{\varepsilon}\right)\left(\mathbf{u}_{0}+\widehat{\mathbf{u}}_{\varepsilon}\right)\right\|_{\mathfrak{G}} \leq \varepsilon\left(\check{\mathcal{C}}_{11}^{(q)}\|\mathbf{q}\|_{\mathfrak{G}}+\widetilde{\mathcal{C}}_{8}^{(r)}\|\mathbf{r}\|_{\mathfrak{G}}\right), \\
&\left\|\mathbf{w}_{\varepsilon}-\left(\mathbf{1}+G_{\eta}^{\varepsilon}\right)\left(\mathbf{w}_{0}+\widehat{\mathbf{w}}_{\varepsilon}\right)\right\|_{\mathfrak{G}} \leq \varepsilon\left(\check{\mathcal{C}}_{10}^{(q)}\|\mathbf{q}\|_{\mathfrak{G}}+\widetilde{\mathcal{C}}_{9}^{(r)}\|\mathbf{r}\|_{\mathfrak{G}}\right), \\
&\left\|\mathbf{v}_{\varepsilon}-\left(\mu^{\varepsilon}\right)^{-1} \mu^{0}\left(\mathbf{v}_{0}+\widehat{\mathbf{v}}_{\varepsilon}\right)\right\|_{\mathfrak{G}} \leq \varepsilon\left(\widehat{\mathcal{C}}_{8}^{(q)}\|\mathbf{q}\|_{\mathfrak{G}}+\check{\mathcal{C}}_{11}^{(r)}\|\mathbf{r}\|_{\mathfrak{G})}\right) \\
&\left\|\mathbf{z}_{\varepsilon}-\left(\mathbf{z}_{0}+\widehat{\mathbf{z}}_{\varepsilon}\right)\right\|_{\mathfrak{G}} \leq \varepsilon\left(\check{\mathcal{C}}_{9}^{(q)}\|\mathbf{q}\|_{\mathfrak{G}}+\check{\mathcal{C}}_{10}^{(r)}\|\mathbf{r}\|_{\mathfrak{G})}\right.
\end{aligned}
$$

The constants in (7.73)-(7.76) depend only on $\|\eta\|_{L_{\infty}},\left\|\eta^{-1}\right\|_{L_{\infty}},\|\mu\|_{L_{\infty}},\left\|\mu^{-1}\right\|_{L_{\infty}}$, and the parameters of the lattice $\Gamma$. 


\section{REFERENCES}

[BaPa] N. S. Bakhvalov and G. P. Panasenko, Homogenization: Averaging processes in periodic media. Mathematical problems in the mechanics of composite materials, "Nauka", Moscow, 1984; English transl., Math. Appl. (Soviet Ser.), vol. 36, Kluwer Acad. Publ. Group, Dordrecht, 1989. MR0797571 (86m:73049) MR:1112788(92d:73002)

[BeLP] A. Bensoussan, J.-L. Lions, and G. Papanicolaou, Asymptotic analysis for periodic structures, Stud. Math. Appl., vol. 5, North-Holland Publishing Co., Amsterdam-New York, 1978. MR.0503330(82h:35001)

[BSu1] M. Sh. Birman and T. A. Suslina, Threshold effects near the lower edge of the spectrum for periodic differential operators of mathematical physics, Systems, Approximations, Singular Integral Operators and Related Topics (Bordeaux, 2000), Oper. Theory Adv. Appl., vol. 129, Birkhäuser, Basel, 2001, pp. 71-107. MR.1882692 (2003f:35220)

[BSu2] - Second order periodic differential operators. Threshold properties and homogenization, Algebra i Analiz 15 (2003), no. 5, 1-108; English transl., St. Petersburg Math. J. 15 (2004), no. 5, 639-714. MR2068790(2005k:47097)

[BSu3] _ - Threshold approximations with corrector for the resolvent of a factorized selfadjoint operator family, Algebra i Analiz 17 (2005), no. 5, 69-90; English transl., St. Petersburg Math. J. 17 (2006), no. 5, 745-762. MR2241423(2008d:47047)

[BSu4] - Homogenization with corrector term for periodic elliptic differential operators, Algebra i Analiz 17 (2005), no. 6, 1-104; English transl., St. Petersburg Math. J. 17 (2006), no. 6, 897-973. MR 2202045 (2006k:35011)

[BSu5] — Homogenization with corrector term for periodic differential operators. Approximation of solutions in the Sobolev class $H^{1}\left(\mathbb{R}^{d}\right)$, Algebra i Analiz 18 (2006), no. 6, 1-130; English transl. in St. Petersburg Math. J. 18 (2007), no. 6, 857-955. MR2307356 (2008d:35008)

[BSu6] - Homogenization of a stationary periodic Maxwell system in the case of constant magnetic permeability, Funktsional. Anal. i Prilozhen. 41 (2007), no. 2, 3-23; English transl. in Funct. Anal. Appl. 41 (2007), no. 2. MR2345036

[H] Q. Huang, Estimates on the generalized Morrey spaces $L_{\varphi}^{2, \lambda}$ and $\mathrm{BMO}_{\psi}$ for linear elliptic systems, Indiana Univ. Math. J. 45 (1996), no. 2, 397-439. MR1414336 (97i:35033)

[ZhKO] V. V. Zhikov, S. M. Kozlov, and O. A. Oleйnik, Homogenization of differential operators, "Nauka", Moscow, 1993; English transl., Springer-Verlag, Berlin, 1994. MR.1318242 (96h:35003a) MR.1329546 (96h: 35003b)

[LaU] O. A. Ladyzhenskaya and N. N. Ural'tseva, Linear and quasilinear equations of elliptic type, "Nauka", Moscow, 1964; English transl., Acad. Press, New York-London, 1968. MR0211073 (35:1955) MR0244627 (39:5941)

[Sa] E. Sanchez-Palencia, Nonhomogeneous media and vibration theory, Lecture Notes in Phys., vol. 127, Springer-Verlag, Berlin-New York, 1980. MR0578345 (82j:35010)

[Su1] T. A. Suslina, On the homogenization of a periodic Maxwell system, Funktsional. Anal. i Prilozhen. 38 (2004), no. 3, 90-94; English transl., Funct. Anal. Appl. 38 (2004), no. 3, 234-237. MR:2095137 (2005g:35017)

[Su2] , Homogenization of a stationary periodic Maxwell system, Algebra i Analiz 16 (2004), no. 5, 162-244; English transl., St. Petersburg Math. J. 16 (2005), no. 5, 863-922. MR2106671 (2005h:35019)

Department of Physics, St. Petersburg State University, Ul'yanovskaya 3, Petrodvorets, 198504 St. Petersburg, Russia

E-mail address: suslina@list.ru

Received 8/FEB/2007

Translated by THE AUTHOR 